\title{
Worldline Path-Integral Representations for Standard Model Propagators and Effective Actions
}

\author{
Somdatta Bhattacharya \\ New Alipore College, Kolkata, West Bengal 700053, India \\ Correspondence should be addressed to Somdatta Bhattacharya; somdatta.bhattacharya@gmail.com
}

Received 15 April 2017; Revised 28 July 2017; Accepted 3 August 2017; Published 19 November 2017

Academic Editor: George Siopsis

Copyright ( 2017 Somdatta Bhattacharya. This is an open access article distributed under the Creative Commons Attribution License, which permits unrestricted use, distribution, and reproduction in any medium, provided the original work is properly cited. The publication of this article was funded by $\mathrm{SCOAP}^{3}$.

\begin{abstract}
We develop the formalism to do worldline calculations relevant for the Standard Model. For that, we first figure out the worldline representations for the free propagators of massless chiral fermions of a single generation and gauge bosons of the Standard Model. Then we extend the formalism to the massive and dressed cases for the fermions and compute the QED vertex. We then go over fermionic one-loop effective actions and anomalies. To our knowledge, in the places where there has been an attempt at deriving the gauge boson propagator, the derivation is somewhat contrived, and we believe our derivation is more straightforward. Moreover, our incorporation of internal degrees of freedom is novel and sports some new features. The derivation of the QED vertex is also new. The treatment of the fermionic one-loop effective actions leads to a particularly economical derivation of chiral anomalies and the gauge anomaly freedom in the Standard Model, improving upon the state of the art in the literature. The appropriate worldline formalism developed thus sets the stage for Standard Model calculations beyond the tree and one-loop cases that incorporate BernKosower type formulae for multiloop amplitudes, relevant for processes at the LHC.
\end{abstract}

\section{Introduction}

That second quantization is equivalent to first quantization when formulated on singular $1 \mathrm{D}$ manifolds/graphs instead of $\mathbf{R}^{1}$ has been known for quite some time, beginning with Feynman [1]. In fact this has been the main raison detre for the formulation of string theory in its present form, where one uses all possible super-Riemann surfaces in place of $1 \mathrm{D}$ manifolds. However, for field theories, it had been mainly existing as a curiosity and a testing ground for ideas to be applied to string theory, until Bern and Kosower ventured to show [2-4] that certain field theory computations were amenable to simplifications when looked upon as infinite tension/field theory limits of certain 4D heterotic string amplitudes. Strassler then proved [5] that the rules that Bern and Kosower had unearthed had a much simpler genesis in worldline formulations of the one-loop effective actions of field theory. That unleashed a whole industry of worldline computations, with generalizations to higher loops [6] and constant external backgrounds [7] among other things, culminating in a review article by Schubert [8], which contains an almost exhaustive list of references up to that point and which much of the subsequent literature also cites. A more recent and pedagogical review is [9].

However most of the literature focussed on worldline path-integral representations of one-loop effective actions for spinning particles, which are formulated on $S^{1}$, and their close cousins, the worldline representations for dressed propagators, that are formulated on segments of $\mathbf{R}^{1}$, did not receive as much attention, mainly because of their lack of utility in relevant computations but also because it was harder to formulate. In fact some of the latter's avatars [10-16] existed even before the Bern-Kosower revolution, and some were formulated later on [17-23]. Particularly, $[13,19,21-23]$ also handled the dressed propagators for the Abelian backgrounds. In any case it remains a relatively less researched subject to this day, compared to the effort devoted to the worldline path-integral representations of effective actions and its derivatives, like loop-level scattering amplitudes, and computations of higher loop beta-functions, whose original references are mostly found in [8]. 
Moreover, most of the derivations for propagators were concerned only with pure spin-half particles, without any internal symmetries of any kind (with the exception of [12] which presciently anticipates some of what is done here). Also, the propagators were often derived not only from the worldline path-integrals per se, but from complicated derivatives thereof. Spin higher than $1 / 2$ was treated in papers like $[7,24]$, but the results were not fully satisfactory in the sense that the connection to the path-integral was not immediate, and arrived at only after certain manipulations. We will see that the connection is more direct than what these papers would have us believe. And again, we will also see how internal symmetries can be incorporated more directly.

The method for incorporating internal symmetries at the first quantized level had been well known for quite some time, starting with [25] and further elaborated upon in [26, 27]. However, their incorporation in worldline path-integrals for propagators [28, 29] and effective actions [30] did not somehow seem appealing until only recently. Moreover, these papers considered only scalar particles with internal symmetries and only included spin-half for the one-loop effective action [30].

Here first of all we demonstrate how to incorporate spin as well as internal symmetries for both fermions and gauge bosons for general $S O(n)$ gauge groups (see $[31,32]$ for related work) and we specialize to the case of $S O(10)$ for the chiral fermions of the SM, for the 16 fermions of the SM in a single generation transform as a spinorial representation of $S O(10)$, upon including a right-handed antineutrino (see [33, 34] for related work). We derive the worldline path-integral representation of the propagators for the chiral fermions of the SM and the appropriate worldline Lagrangian includes worldline fermions pertaining to spin-half, as well as others pertaining to the $S O(10)$ group.

We also work out the path-integral representation for the $S O(n)$ gauge boson propagator. The $S U(3)$ gauge boson propagator can be derived from the $S O(6)$ one, of which it is a subgroup (with certain caveats, cf. end of Section 5), and the $S U(2)$ one can be obtained from the $S O(3)$ one, of which it is a double cover. Or if one wishes to derive everything starting from $S O(10)$, one can simply choose $n=10$. All these propagators are derived in the so-called coherent state bases, to be explained below, and it is straightforward to transform them to the index bases as we will see.

The worldline actions involved for both spin-half and spin-one have local reparametrization symmetries and supersymmetries, which have to be fixed by choosing gauges, and that necessarily entails Faddeev-Popov ghosts. Here, in the initial derivations we simply gauge-fix and throw away the gauge volumes without any justifications, reserving the proper derivation for later, wherein we do a full Hamiltonian BRST analysis. In any case, as is well known and we find ourselves, the ghosts decouple trivially for the case of the open worldline.

It is easy to see how to extend the analysis for the case of the graviton, but we do not do that here. Throughout, we make use of a technique outlined in [29], adapting the analysis there in terms of internal symmetry worldline fermions, to the spinning case here. We show that the propagators are related directly to the worldline path-integrals and not to any derivatives of it, albeit in the coherent state bases.

After working out the free massless propagators, we concentrate on the dressed massive propagators and as an application derive the QED vertex by dressing the electron propagator by one photon. This simple result proves formidable to derive and it is gratifying that the various terms cancel subtly among each other to give the right result.

Our next quest becomes derivation of fermionic one-loop effective actions and anomalies, both chiral and gauge, and we demonstrate gauge anomaly freedom in the SM in an especially economic fashion. Since the Atiyah-Singer index theorem is related to the chiral anomaly, the methods used here can be looked upon as yet another derivation of the theorem, when the background is nongravitational. These anomalies were first formulated in terms of worldline pathintegrals in [35] and were put on more rigorous footing in [36] and the analysis finally culminated in a book [37]. One can compare the methods in these references with the methods here to appreciate the economy of arguments and calculational steps.

One of the main aims of this paper is getting the quantum mechanical models whose wave-functions are the fields of the SM. Since the internal symmetry worldline fermions get transformed into fermionic creation and annihilation operators upon quantization (cf. Sections 5 and 6) the different states in a spin-half or spin-one sector can simply be seen as excitations over ground states. Taking the cue from here, one might suppose that the other generations of the SM also have a similar genesis; namely, they too might be built upon the states of the first generation via the action of even more creation operators. We have been exploring this issue and have found that 6 more creation and annihilation operators are needed, which can be made to lead to 3 chiral and one vector-like generations upon imposition of GSO-like projection conditions. This necessarily enlarges the symmetry group from $S O(10)$ to $S O(16)$ and this then has ramifications for the scalar sector. The scalar sector in an $S O(10)$ GUT is extremely rich, and there are certain advantages in making it even richer, like reducing the number of Yukawa couplings to just one with the SO(10) Yukawa couplings arising from spontaneous symmetry breaking from $S O(16)$ to $S O(10)$. A major problem for $S O(16)$ is that its representations are known to be vector-like; however, that can be taken care of by the GSO-like projection operators on the spectrum of states, which enforces the condition that differently handed states that are exactly similar otherwise cannot both survive the projection.

What we do here is mainly developing a formalism, to be applied to more involved processes than the ones considered here. So though there is no new physical result, we would like to stress that the formalism uncovered is novel, as is evident from the economy of arguments and calculational steps mentioned before, among other things. In particular, the thing that is primarily uncovered is the immediacy and simplicity of the coherent state formalism when interpreted in the way we have followed here.

The rest of the paper is organized as follows. In Sections 2 and 3, we derive the worldline representations of the massless 
propagators for bare spin-half and spin-one particles, respectively, which we believe are novel in themselves as they differ and improve upon the derivations present in the literature. We then do a full Hamiltonian BRST treatment for the spinone case in Section 4. In Sections 5 and 6, we show how to incorporate internal symmetries into the mix, deriving the propagators for the SM gauge bosons and chiral fermions, respectively. In Section 7 we extend the formalism to massive dressed propagators for the spin-half cases and in Section 8, we use the formalism developed to compute the QED vertex. Then we launch into the topic of fermionic one-loop effective actions in Section 9, specializing to the case of QCD in Section 10, and derive the chiral anomaly and prove gauge anomaly freedom of the SM in Section 11. Finally we conclude in Section 12. There are three appendices that elaborate upon certain calculational steps.

\section{Propagator for the Spin-Half Particle}

A spin-half particle is in reality a $1 \mid 1$-dimensional object parametrized by a bosonic parameter $\tau$ and a fermionic parameter $\theta$, and the embedding of these coordinates in spacetime is given, instead of $x^{\mu}(\tau)$, by $X^{\mu}(\tau, \theta)$, which embeds as

$$
X^{\mu}(\tau, \theta)=x^{\mu}(\tau)+\theta \psi^{\mu}(\tau)
$$

which in other words means that the electron sees a 4 4dimensional super-spacetime. The fermionic coordinates $\psi^{\mu}$ are nilpotent, so the fermionic submanifold is infinitesimal and compact; however, it is fibered over spacetime and, together, they form what is known as the Clifford-bundle, once the theory is quantized and $\psi^{\mu}$ become the Dirac matrices, $\gamma^{\mu}$. [38-41]

The Lagrangian for a single massless electron is given by

$$
L=p^{\mu} \dot{x}_{\mu}+\frac{i}{2} \psi^{\mu} \dot{\psi}_{\mu}-\frac{1}{2} e p^{\mu} p_{\mu}-i \beta \psi^{\mu} p_{\mu}
$$

We are working in the metric conventions $(+---)$. This Lagrangian has local reparametrization symmetry, generated by $(1 / 2) p^{2}$, and local worldline supersymmetry, generated by $\psi^{\mu} p_{\mu}$. It is convenient to transform the "Majorana" fermions $\psi^{\mu}$ in the kinetic term to "Dirac" fermions as follows:

$$
L=p^{\mu} \dot{x}_{\mu}-\frac{1}{2} e p^{\mu} p_{\mu}-i \beta \psi^{\mu} p_{\mu}+i \bar{\psi}_{i} \dot{\psi}_{i}
$$

where, by the doubling trick,

$$
\begin{aligned}
& \psi^{0}=\frac{1}{\sqrt{2}}\left(\bar{\psi}_{1}+\psi_{1}\right), \\
& \psi^{3}=\frac{1}{\sqrt{2}}\left(\bar{\psi}_{1}-\psi_{1}\right), \\
& \psi^{2}=\frac{i}{\sqrt{2}}\left(\psi_{2}+\bar{\psi}_{2}\right), \\
& \psi^{1}=\frac{1}{\sqrt{2}}\left(\bar{\psi}_{2}-\psi_{2}\right) .
\end{aligned}
$$

The Majorana indices on the LHS are the ones in (2) and those on the right are the Dirac ones in (3). Upon Dirac quantization the barred objects in (3) become creation operators while the unbarred ones become annihilation operators, satisfying the commutation relations

$$
\left\{\bar{\psi}_{i}, \psi_{j}\right\}=\delta_{i j} .
$$

The creation operators act on a common vacuum to produce the fermionic subspace of the physical states that are precisely the spin-1/2 states. They are of the form

$$
\begin{gathered}
|0\rangle, \\
\widehat{\bar{\psi}}_{1}|0\rangle, \\
\widehat{\bar{\psi}}_{2}|0\rangle, \\
\widehat{\bar{\psi}}_{1} \widehat{\bar{\psi}}_{2}|0\rangle,
\end{gathered}
$$

where $|0\rangle$ collectively denotes the vacua of species 1 and 2 . Now, if one assigns the following indices to the above states,

$$
\begin{aligned}
& R 1 \longrightarrow|0\rangle, \\
& L 1 \longrightarrow \widehat{\bar{\psi}}_{1}|0\rangle, \\
& L 2 \longrightarrow \widehat{\bar{\psi}}_{2}|0\rangle, \\
& R 2 \longrightarrow \widehat{\bar{\psi}}_{1} \hat{\bar{\psi}}_{2}|0\rangle,
\end{aligned}
$$

where $R$ and $L$ denote right- and left-handed, the matrix elements of $\sqrt{2} \widehat{\psi}^{\mu}$ with respect to the above states yield just the matrix elements of the Dirac matrices $\gamma^{\mu}$ in the chiral representation; namely,

$$
\gamma^{\mu}=\left(\begin{array}{cc}
0 & \sigma^{\mu} \\
\bar{\sigma}^{\mu} & 0
\end{array}\right) .
$$

The above stems from converting $\sqrt{2} \widehat{\psi}^{\mu}$ to the Dirac basis and treating them as creation annihilation operators. The full wave-function is a direct product of elements of the bosonic Hilbert space and the fermionic Hilbert space above and together they form the components of a Dirac spinor.

Now the theory has constraints, obtained from (3) by varying with respect to the auxiliary fields $e$ and $\beta$ and they are $p^{2}=0$ and $\psi^{\mu} p_{\mu}=0$. The representation of a Dirac spinor is

$$
\begin{aligned}
|\alpha\rangle= & \left(\begin{array}{c}
\left|\alpha_{L, 1}\right\rangle \\
\left|\alpha_{L, 2}\right\rangle \\
\left|\alpha_{R, 1}\right\rangle \\
\left|\alpha_{R, 2}\right\rangle
\end{array}\right) \\
= & \left|\alpha_{R, 1}\right\rangle|0\rangle+\left|\alpha_{L, 1}\right\rangle \widehat{\bar{\psi}}_{1}|0\rangle+\left|\alpha_{L, 2}\right\rangle \widehat{\bar{\psi}}_{2}|0\rangle \\
& +\left|\alpha_{R, 2}\right\rangle \hat{\bar{\psi}}_{1} \hat{\bar{\psi}}_{2}|0\rangle .
\end{aligned}
$$


As a consequence of the above facts the constraint $\sqrt{2} \widehat{\psi}^{\mu} p_{\mu}$ acting on the above state yields the Dirac equation

$$
\left\langle x\left|\gamma^{\mu} p_{\mu}\right| \alpha\right\rangle=-i \gamma^{\mu} \partial_{\mu} \alpha(x)=0 .
$$

There is an alternate basis of states, formed by the fermionic coherent states, which satisfy

$$
\widehat{\psi}_{i}\left|\psi_{1}\right\rangle=\psi_{1 i}\left|\psi_{1}\right\rangle
$$

where $\psi_{1 i}$ is a Grassmann number. This means the above state is of the form

$$
\left|\psi_{1}\right\rangle=e^{\overline{\bar{\psi}}_{i} \psi_{1 i}}|0\rangle
$$

because of the anticommutation relations between $\widehat{\psi}_{i}$ and $\widehat{\bar{\psi}}_{i}$. Bra states $\left\langle\bar{\psi}_{2}\right|$ are of the form

$$
\left\langle\bar{\psi}_{2}\right|=\langle 0| e^{\bar{\psi}_{2 i} \widehat{\psi}_{i}}
$$

where they satisfy

$$
\left\langle\bar{\psi}_{2}\right| \bar{\psi}_{2 i}=\left\langle\bar{\psi}_{2}\right| \widehat{\bar{\psi}}_{i}
$$

This in particular means

$$
\left\langle\bar{\psi}_{2} \mid \psi_{1}\right\rangle=e^{\left(\bar{\psi}_{2 i} \psi_{1 i}\right)}
$$

They also satisfy the properties

$$
\begin{aligned}
& \left\langle\bar{\psi}_{2}\right| \widehat{\psi}_{i}=\frac{\partial}{\partial \bar{\psi}_{2 i}}\left\langle\bar{\psi}_{2}\right|, \\
& \widehat{\bar{\psi}}_{i}\left|\psi_{1}\right\rangle=-\frac{\partial}{\partial \psi_{1 i}}\left|\psi_{1}\right\rangle .
\end{aligned}
$$

Let us now see whether the path-integral of the action (3) reproduces the Dirac propagator. One starts with the following path-integral form:

$$
\begin{aligned}
& \int_{x_{\mu}(0)=x_{\mu}}^{x_{\mu}(1)=x_{\mu}^{\prime}} \mathscr{D} x \int_{\psi_{i}(0)=\psi_{1 i}}^{\bar{\psi}_{i}(1)=\bar{\psi}_{2 i}} \mathscr{D} \psi \mathscr{D} \bar{\psi} \\
& \quad \cdot \int \frac{\mathscr{D} \beta \mathscr{D} e}{\operatorname{Vol}(\mathrm{Sym})} \mathscr{D} p e^{i \int_{0}^{1} d t\left(p^{\mu} \dot{x}_{\mu}-(1 / 2) e p^{\mu} p_{\mu}-i \beta \psi^{\mu} p_{\mu}+i \bar{\psi}_{i} \dot{\psi}_{i}\right)+\bar{\psi}_{i} \psi_{i}(1),}
\end{aligned}
$$

where the gauge volumes of the reparametrization symmetry and supersymmetry of the action have been divided and we have set certain boundary conditions for $x$ 's and $\psi$ 's and the choice of the boundary conditions will become clearer later. $e$ and $\beta$ are like gauge fields that transform under these symmetries and one has to gauge-fix them to avoid overcounting by restricting them to single gauge slices. The integral over the gauge-orbits would then cancel the volume factor. This would necessarily entail Faddeev-Popov ghosts, and we address the issue in Section 4, albeit in the context of the spin-1 particle. As we will see there, the dependence on the ghosts becomes trivial and this is related to the fact that, for an open line, the FP determinant becomes trivial on gauge fixing $e$ to a constant and so does it for $\beta$. A similar analysis is applicable to the spin-half case as well. For the present we simply assume that, upon gauge fixation, one can simply throw away the gauge volumes. The boundary term is necessary in order for the field equation for $\psi_{i}$ to be given by $(d / d t) \bar{\psi}_{i}=0$ without incurring extra boundary terms ${ }^{1}$. First we simultaneously fix $e$ to $2 T$ and $\beta$ to $\theta$. Then upon integrating out $p_{\mu}$ one gets

$$
\begin{aligned}
& \int d T d \theta \int_{x_{\mu}(0)=x_{\mu}}^{x_{\mu}(1)=x_{\mu}^{\prime}} \mathscr{D} x \\
& \cdot \int_{\psi_{i}(0)=\psi_{1 i}}^{\bar{\psi}_{i}(1)=\bar{\psi}_{2 i}} \mathscr{D} \psi \mathscr{D} \bar{\psi} e^{i \int_{0}^{1} d t\left((1 / 4 T) \dot{x}^{2}+i \bar{\psi}_{i} \dot{\psi}_{i}-i(\theta / 2 T)\left(\bar{\psi}_{i} \dot{x}_{i}+\psi_{i} \dot{\bar{x}}_{i}\right)\right)+\bar{\psi}_{i} \psi_{i}(1)},
\end{aligned}
$$

where we have used

$$
\psi^{\mu} \dot{x}_{\mu}=\bar{\psi}_{i} \dot{x}_{i}+\psi_{i} \dot{\bar{x}}_{i}
$$

where $x_{i}\left(\bar{x}_{i}\right)$ have the same relations to $x_{\mu}$ as $\psi_{i}\left(\bar{\psi}_{i}\right)$ have to $\psi_{\mu}$ (cf. (4)).

Upon Wick rotating $t$, one gets

$$
\begin{aligned}
& \int d T d \theta \int_{x_{\mu}(0)=x_{\mu}}^{x_{\mu}(1)=x_{\mu}^{\prime}} \mathscr{D} x \\
& \cdot \int_{\psi_{i}(0)=\psi_{1 i}}^{\bar{\psi}_{i}(1)=\bar{\psi}_{2 i}} \mathscr{D} \psi \mathscr{D} \bar{\psi} e^{\int_{0}^{1} d t\left(-(1 / 4 T) \dot{x}^{2}-\bar{\psi}_{i} \dot{\psi}_{i}+(\theta / 2 T)\left(\bar{\psi}_{i} \dot{x}_{i}+\psi_{i} \dot{\bar{x}}_{i}\right)\right)+\bar{\psi}_{i} \psi_{i}(1)} .
\end{aligned}
$$

Now, let us perform the integral over $\theta$. We get

$$
\begin{aligned}
& \int d T \int_{x_{\mu}(0)=x_{\mu}}^{x_{\mu}(1)=x_{\mu}^{\prime}} \mathscr{D} \int_{\psi_{i}(0)=\psi_{1 i}}^{\bar{\psi}_{i}(1)=\bar{\psi}_{2 i}} \mathscr{D} \psi \mathscr{D} \bar{\psi} \\
& \cdot \int d t \frac{1}{2 T}\left(\bar{\psi}_{i} \dot{x}_{i}+\psi_{i} \dot{\bar{x}}_{i}\right) e^{\int_{0}^{1} d t\left(-(1 / 4 T) \dot{x}^{2}-\bar{\psi}_{i} \dot{\psi}_{i}\right)+\bar{\psi}_{i} \psi_{i}(1)} .
\end{aligned}
$$

Let us then concentrate on the fermionic integral. The free fermionic path-integral becomes [29]

$$
\int_{\psi_{i}(0)=\psi_{1 i}}^{\bar{\psi}_{i}(1)=\bar{\psi}_{2 i}} \mathscr{D} \psi \mathscr{D} \bar{\psi} e^{\int_{0}^{1} d t\left(-\bar{\psi}_{i} \dot{\psi}_{i}\right)+\bar{\psi}_{i} \psi_{i}(1)}=e^{\bar{\psi}_{2 i} \psi_{1 i}} .
$$

When one evaluates the correlators in (21), this part would be omnipresent, as is usual, as the normalization factor (cf. Appendix A). Now in evaluating the fermionic correlators in (21), we first split the fields into background and fluctuations

$$
\begin{gathered}
\psi_{i}\left(t^{\prime}\right)=\psi_{1 i}+\kappa_{i}\left(t^{\prime}\right), \quad \kappa_{i}(0)=0, \\
\bar{\psi}_{i}(t)=\bar{\psi}_{2 i}+\bar{\kappa}_{i}(t), \quad \bar{\kappa}_{i}(1)=0 .
\end{gathered}
$$

The kinetic action for the fluctuations $\int_{0}^{1} d T \bar{\kappa}_{i} \dot{\kappa}_{i}$ can be inverted to give the propagator

$$
\left\langle\kappa_{i}(t) \bar{\kappa}_{j}\left(t^{\prime}\right)\right\rangle=\delta_{i j} \theta\left(t-t^{\prime}\right) .
$$

The fermionic part can thus be written as

$$
\begin{aligned}
& \frac{1}{2 T} e^{\bar{\psi}_{2 i} \psi_{1 i}}\left\langle\int d t \left[\left(\bar{\psi}_{2 i}+\bar{\kappa}_{i}(t)\right) \dot{x}_{i}\right.\right. \\
& \left.\left.+\dot{\bar{x}}_{i}\left(\psi_{1 i}+\kappa_{i}\left(t^{\prime}\right)\right)\right]\right\rangle,
\end{aligned}
$$


where the one-point functions are with respect to the action for the fluctuations. Now the one-point functions for $\bar{\kappa}$ 's and $\kappa$ 's vanish and using

$$
\int_{\mathcal{K}_{i}(0)=0}^{\bar{\kappa}_{i}(1)=0} \mathscr{D} \mathcal{K} \mathscr{D} \bar{\kappa} e^{\int_{0}^{1} d t\left(-\bar{\kappa}_{i} \dot{\kappa}_{i}\right)}=1
$$

one is left with

$$
\int_{0}^{\infty} d T \frac{1}{2 T} e^{\bar{\psi}_{2 i} \psi_{1 i}}\left\langle\int d t\left[\left(\bar{\psi}_{2 i}\right) \dot{x}_{i}+\dot{\bar{x}}_{i}\left(\psi_{1 i}\right)\right]\right\rangle
$$

where now the one-point functions are of $\dot{x}$ 's and are with respect to the action for $x$ (cf. (18)). Again, $x$ 's can be separated into background and fluctuations

$$
x_{i}(t)=x_{i}(0)+\left(x_{i}(1)-x_{i}(0)\right) t+y_{i}(t)
$$

with a similar expansion for $\bar{x}$ 's, where $y(0)=y(1)=0$, and one has

$$
\dot{x}_{i}(t)=\left(x_{i}(1)-x_{i}(0)\right)+\dot{y}_{i}(t)
$$

and the $x$ action becomes

$$
\begin{gathered}
-\int d t \frac{1}{4 T}\left[(x(1)-x(0))^{2}+2(x(1)-x(0))_{i} \dot{y}_{i}\right. \\
\left.+\dot{y}^{2}\right]=-\int d t \frac{1}{4 T}\left[(x(1)-x(0))^{2}+\dot{y}^{2}\right] .
\end{gathered}
$$

Again, the $\dot{y}$ one-point function vanishes and upon substituting the values for $x(1)$ and $x(0)$, and taking into account the contribution from the free integral over $y$ 's, we get that the $\dot{x}$ one-point function is

$$
\left\langle\dot{x}_{i}(t)\right\rangle=\frac{1}{(4 \pi T)^{2}}\left(x_{i}^{\prime}-x_{i}\right) e^{-\left(x^{\prime}-x\right)^{2} / 4 T} .
$$

Thus (27) becomes, upon incorporating similar effects for the $\bar{x}$ part and performing the $T$ integral,

$$
e^{\left(\bar{\psi}_{2 i} \psi_{1 i}\right)} \frac{\left(\left(\bar{x}_{i}^{\prime}-\bar{x}_{i}\right) \psi_{1 i}+\left(x_{i}^{\prime}-x_{i}\right) \bar{\psi}_{2 i}\right)}{2 \pi^{2}\left(x^{\prime}-x\right)^{4}}
$$

where one has used

$$
\begin{gathered}
\left(\frac{1}{2 \pi}\right)^{2} \int_{0}^{\infty} \frac{d T}{2 T}\left(\frac{1}{2 T}\right)^{2} e^{-\left(x^{\prime}-x\right)^{2} / 4 T} \\
=\frac{1}{2 \pi^{2}} \int_{0}^{\infty} d \sigma \sigma e^{-\sigma\left(x^{\prime}-x\right)^{2}}
\end{gathered}
$$

upon transforming $4 T \rightarrow \sigma^{-1}$, which finally becomes

$$
=\frac{1}{2 \pi^{2}\left(x^{\prime}-x\right)^{4}} \text {. }
$$

Throughout, up to Section 6, we are working with $\left(x^{\prime}-\right.$ $x)^{2} \geq 0$, since, otherwise, integrals such as in (33) blow up as $\sigma \rightarrow \infty$. In Sections 7 and 8 we will be working with $\left(x^{\prime}-\right.$ $x)^{2} \leq 0$, as will be clarified there. Beyond Section 8 , where we are working with one-loop amplitudes, such considerations do not apply. But now notice that (32) is precisely

$$
\left\langle\bar{\psi}_{2}\left|\frac{\left(\bar{x}_{i}^{\prime}-\bar{x}_{i}\right) \widehat{\psi}_{i}+\left(x_{i}^{\prime}-x_{i}\right) \hat{\bar{\psi}}_{i}}{2 \pi^{2}\left(x^{\prime}-x\right)^{4}}\right| \psi_{1}\right\rangle,
$$

where $\left|\psi_{1}\right\rangle$ and $\left\langle\bar{\psi}_{2}\right|$ are the coherent states defined above. Now expression (35) is equal to

$$
\left\langle\bar{\psi}_{2}\left|\frac{\left(\left(x_{\mu}^{\prime}-x_{\mu}\right) \widehat{\psi}^{\mu}\right)}{2 \pi^{2}\left(x^{\prime}-x\right)^{4}}\right| \psi_{1}\right\rangle
$$

because of (19). This can be written as

$$
-\left\langle\bar{\psi}_{2}\left|\widehat{\psi}^{\mu} \partial_{\mu}^{\prime} \frac{1}{4 \pi^{2}\left(x^{\prime}-x\right)^{2}}\right| \psi_{1}\right\rangle
$$

where the prime on the derivative denotes differentiation with respect to $x^{\prime}$. But as we saw above, the operator $\hat{\psi}^{\mu}$ is precisely $\gamma^{\mu} / \sqrt{2}$ when acting on the basis (7), which is a complementary basis to the overcomplete coherent state one, and, transforming to this basis, one has

$$
-\frac{1}{\sqrt{2}}\left\langle i\left|\gamma^{\mu} \partial_{\mu}^{\prime} \frac{1}{4 \pi^{2}\left(x^{\prime}-x\right)^{2}}\right| j\right\rangle,
$$

where $i$ and $j$ stand for the four components of (7). But this is precisely equal to

$$
\frac{i}{\sqrt{2}}\left\langle x^{\prime}, i\left|\frac{\gamma \cdot p}{p^{2}}\right| x, j\right\rangle=\frac{i}{\sqrt{2}}\left\langle x^{\prime}, i\left|\frac{1}{\gamma \cdot p}\right| x, j\right\rangle .
$$

Summarizing,

$$
\begin{aligned}
& \frac{i}{\sqrt{2}}\left\langle x^{\prime}, \bar{\psi}_{2}\left|\frac{1}{\gamma \cdot p}\right| x, \psi_{1}\right\rangle=\int_{x_{\mu}(0)=x_{\mu}}^{x_{\mu}(1)=x_{\mu}^{\prime}} \mathscr{D} x \int_{\psi_{i}(0)=\psi_{1 i}}^{\bar{\psi}_{i}(1)=\bar{\psi}_{2 i}} \mathscr{D} \psi \mathscr{D} \bar{\psi} \\
& \cdot \int \frac{\mathscr{D} \beta \mathscr{D} e}{\operatorname{Vol}(\mathrm{Sym})} \mathscr{D} p e^{i \int_{0}^{1} d t\left(p^{\mu} \dot{x}_{\mu}-(1 / 2) e p^{\mu} p_{\mu}-i \beta \psi^{\mu} p_{\mu}+i \bar{\psi}_{i} \dot{\psi}_{i}\right)+\bar{\psi}_{i} \psi_{i}(1)},
\end{aligned}
$$

where $\gamma$ means the usual gamma matrix when acting on the Dirac basis and an oscillation operator when acting on the coherent state basis.

Thus we see that the massless Dirac propagator can be given a path-integral representation given by the above expression. Now we claim that this entire derivation essentially boils down to the above expression being equivalent to

$$
\int_{0}^{\infty} d T \int d \theta\left\langle X^{\prime}\left|e^{-T H-i \theta Q}\right| X\right\rangle,
$$

where $H=p^{2}$ and $Q=\widehat{\psi} \cdot p$ and where each of these is now an operator. $|X\rangle$ and $\left\langle X^{\prime}\right|$ stand for

$$
\begin{aligned}
& |x\rangle \otimes\left|\psi_{1}\right\rangle, \\
& \left\langle x^{\prime}\right| \otimes\left\langle\bar{\psi}_{2}\right|,
\end{aligned}
$$


respectively. Upon integrating over the $T$ and $\theta$, one gets

$$
\begin{aligned}
i\left\langle X^{\prime}\left|\frac{Q}{H}\right| X\right\rangle & =i\left\langle X^{\prime}\left|\frac{\widehat{\psi} \cdot p}{p^{2}}\right| X\right\rangle \\
& =\frac{i}{\sqrt{2}}\left\langle x^{\prime}, \bar{\psi}_{2}\left|\frac{1}{\gamma \cdot p}\right| x, \psi_{1}\right\rangle,
\end{aligned}
$$

where $\gamma$ has the same meaning as that above, thus vindicating our claim. We will make use of similar equivalences later. In fact (41) has been the starting point for many of the earlier treatments [11-16, 19-23] and they have proceeded in the opposite route from this to the path-integral form. Here we have shown how to arrive at the above starting from the pathintegral.

We would like to remark that our treatment is closest in spirit to $[12,14]$, and we will see how to extend the formalism to the massive and dressed case in Section 7, with its vindication in Section 8, where we derive the QED vertex.

\section{Propagator for the Spin-One Particle}

Let us now come to the spin-1 particle. It is a 1 | 2 dimensional object with embedding in spacetime given by $X^{\mu}(\tau, \theta, \bar{\theta})$. Each $x^{\mu}$ has two fermionic superpartners, $\bar{\psi}^{\mu}$ and $\psi^{\mu}$. The expansion is

$$
X^{\mu}(\tau, \theta, \bar{\theta})=x^{\mu}(\tau)+\theta \bar{\psi}^{\mu}(\tau)+\bar{\theta} \psi^{\mu}(\tau)+\theta \bar{\theta} b^{\mu}(\tau)
$$

$b^{\mu}$ can be eliminated by its equation of motion as it appears in the action without a kinetic term. The locally supersymmetric $\mathcal{N}=2$ worldline Lagrangian is given by $[42,43]$

$$
\begin{aligned}
L= & p^{\mu} \dot{x}_{\mu}+i \bar{\psi}^{\mu} \dot{\psi}_{\mu}-\frac{1}{2} e p^{\mu} p_{\mu}-i \beta \bar{\psi}^{\mu} p_{\mu}-i \bar{\beta} \psi^{\mu} p_{\mu} \\
& -a \bar{\psi}^{\mu} \psi_{\mu}+s a,
\end{aligned}
$$

where there are additional terms in comparison to the $\mathscr{N}=1$ Lagrangian in (2), whose significance is that they project the physical state space onto a subspace of specific $N_{\overline{\bar{\psi}}}[42,43]$, whose meaning will become clearer below.

$a$ is a worldline $U(1)$ gauge field and $\bar{\psi}^{\mu} \psi_{\mu}$ generates a $U(1)$ R-symmetry. In particular, choosing $s=0$ projects to the $N_{\widehat{\bar{\psi}}}=2$ sector, the one relevant for two-forms, the antisymmetric tensor corresponding to $F_{\mu \nu} \bar{\psi}^{\mu} \bar{\psi}^{\nu}$ in this case. This is because once the fields are quantized, $\psi$ 's become operators, and a term like $\widehat{\bar{\psi}}^{\mu} \widehat{\psi}_{\mu}$ becomes ambiguous with respect to its ordering. To resolve this, one has to antisymmetrize it and define it as $(1 / 2)\left(\widehat{\bar{\psi}}^{\mu} \widehat{\psi}_{\mu}-\widehat{\psi}_{\mu} \hat{\bar{\psi}}^{\mu}\right)$, so that it picks up a normal ordering constant, upon being normal ordered, of value 2 . So the operator that acts on physical states $\Phi(x, \bar{\psi})$ is of the form $\widehat{\bar{\psi}}^{\mu} \widehat{\psi}_{\mu}-2=\bar{\psi}^{\mu}\left(\partial / \partial \bar{\psi}^{\mu}\right)-2$, and it projects onto 2 -forms, when $s=0$. The sa term is evidently a Chern-Simmons term, appropriate for a 1D action.

Now it is well known that quantization of the above Lagrangian gives rise to the free Maxwell equations for $F_{\mu \nu}$ $[42,43]$. Thus the path-integral ought to give rise to the correlator for it. Upon Euclideanisation of the action, one gets the following path-integral form:

$$
\int_{x_{\mu}(0)=x_{\mu}}^{x_{\mu}(1)=x_{\mu}^{\prime}} \mathscr{D} x \int_{\psi_{\mu}(0)=\psi_{\mu 1}}^{\bar{\psi}_{\mu}(1)=\bar{\psi}_{\mu 2}} \mathscr{D} \psi \mathscr{D} \bar{\psi} \times \int \frac{\mathscr{D} a \mathscr{D} \beta \mathscr{D} \bar{\beta} \mathscr{D} e}{\operatorname{Vol}(\operatorname{Sym})} \mathscr{D} p e^{\int_{0}^{1} d t\left(i p^{\mu} \dot{x}_{\mu}-\bar{\psi}_{\mu} \dot{\psi}_{\mu}-(1 / 2) e p^{\mu} p_{\mu}-i \beta \bar{\psi}^{\mu} p_{\mu}-i \bar{\beta} \psi^{\mu} p_{\mu}-i a \bar{\psi}^{\mu} \psi_{\mu}\right)+\bar{\psi}_{\mu} \psi_{\mu}(1)}
$$

First we simultaneously fix $e$ to $2 T, \beta$ to $\theta$, and $\bar{\beta}$ to $\bar{\theta}$ and $a$ to a constant which ranges from 0 to $2 \pi$, since it is a $U(1)$ gauge field and hence compact (large gauge transformations lead us to identify $\phi$ with $\phi+2 n \pi$, where $\left.\phi=\int_{0}^{1} d t a(t)[43]\right)$.
This constant we continue to call $a$ and its associated FaddeevPopov determinant is a constant independent of $a$ which can be taken as $(2 \pi)^{-1}$. Then as before, we first integrate out $p_{\mu}$. It begets

$$
\int d T d \theta d \bar{\theta} \frac{d a}{(2 \pi)} \int_{x_{\mu}(0)=x_{\mu}}^{x_{\mu}(1)=x_{\mu}^{\prime}} \mathscr{D} x \int_{\psi_{\mu}(0)=\psi_{\mu 1}}^{\bar{\psi}_{\mu}(1)=\bar{\psi}_{\mu 2}} \mathscr{D} \psi \mathscr{D} \bar{\psi} e^{\int_{0}^{1} d t\left(-(1 / 4 T) \dot{x}^{2}-\bar{\psi}_{\mu} \dot{\psi}_{\mu}+(\theta / 2 T) \bar{\psi}^{\mu} \dot{x}_{\mu}+(\bar{\theta} / 2 T) \psi^{\mu} \dot{x}_{\mu}-i(a-i(\bar{\theta} \theta / 2 T)) \bar{\psi}^{\mu} \psi_{\mu}\right)+\bar{\psi}_{\mu} \psi_{\mu}(1)}
$$

We will now evaluate this using the methods of [29]. First of all let us perform the integrals over $\theta$ and $\bar{\theta}$. We get

$$
\begin{aligned}
& \int d T \frac{d a}{(2 \pi)} \int_{x_{\mu}(0)=x_{\mu}}^{x_{\mu}(1)=x_{\mu}^{\prime}} \mathscr{D} x \\
& \quad \cdot \int_{\psi_{\mu}(0)=\psi_{\mu 1}}^{\bar{\psi}_{\mu}(1)=\bar{\psi}_{\mu 2}} \mathscr{D} \psi \mathscr{D} \bar{\psi}\left(\int d t d t^{\prime} \frac{1}{4 T^{2}} \bar{\psi}^{\mu} \dot{x}_{\mu}(t) \psi^{\nu} \dot{x}_{\nu}\left(t^{\prime}\right)\right.
\end{aligned}
$$

$$
\begin{aligned}
& \left.-\int d t \frac{1}{2 T} \bar{\psi}^{\mu} \psi_{\mu}\right) \\
& \times\left(e^{\int_{0}^{1} d t\left(-(1 / 4 T) \dot{x}^{2}-\bar{\psi}_{\mu} \dot{\psi}_{\mu}-i a \bar{\psi}^{\mu} \psi_{\mu}\right)+\bar{\psi}_{\mu} \psi_{\mu}(1)}\right) .
\end{aligned}
$$

Let us concentrate on the fermionic integral. First of all it is convenient to twist the fermions in the following manner: 


$$
\begin{aligned}
& \psi(t) \longrightarrow \psi(t) e^{i a t}, \\
& \bar{\psi}(t) \longrightarrow \bar{\psi}(t) e^{-i a t},
\end{aligned}
$$

so that the free part of the fermionic path-integral becomes [29] (see Appendix B)

$$
\begin{aligned}
& \int_{\psi_{\mu}(0)=\psi_{\mu 1}}^{\bar{\psi}_{\mu}(1)=e^{-i a} \bar{\psi}_{\mu 2}} \mathscr{D} \psi \mathscr{D} \bar{\psi} e^{\int_{0}^{1} d t\left(-\bar{\psi}_{\mu} \dot{\psi}_{\mu}\right)+\bar{\psi}_{\mu} \psi_{\mu}(1)} \\
& =e^{e^{-i a} \bar{\psi}_{\mu 2} \psi_{\mu 1}+2 i a} .
\end{aligned}
$$

As before, when one evaluates the correlators in (48), this part would be omnipresent, as the normalization factor. The extra factor of $2 \mathrm{ia}$ is generated because, in particular, the values of $m$ and $n / 2$ in (B.2) are both 2 in this case. Now in evaluating the fermionic part in (48), again we first split the fields into background and fluctuations

$$
\begin{aligned}
& \psi_{\mu}\left(t^{\prime}\right)=\psi_{\mu 1}+\kappa_{\mu}\left(t^{\prime}\right), \quad \kappa(0)=0, \\
& \bar{\psi}_{\mu}(t)=e^{-i a} \bar{\psi}_{\mu 2}+\bar{\kappa}_{\mu}(t), \quad \bar{\kappa}_{\mu}(1)=0 .
\end{aligned}
$$

The kinetic action for the fluctuations $\int_{0}^{1} d T \bar{\kappa}^{\mu} \dot{\kappa}_{\mu}$ can be inverted to give the propagator

$$
\left\langle\kappa_{\mu}(t) \bar{\kappa}_{\nu}\left(t^{\prime}\right)\right\rangle=\eta_{\mu \nu} \theta\left(t-t^{\prime}\right) .
$$

The fermionic part can thus be written as

$$
\begin{aligned}
& \int_{0}^{2 \pi} \frac{d a}{2 \pi} e^{2 i a+e^{-i a} \bar{\psi}_{2} \cdot \psi_{1}}\left\langle\int d t d t ^ { \prime } \left[\left(e^{-i a} \bar{\psi}_{2}^{\mu}+\bar{\kappa}^{\mu}(t)\right)\right.\right. \\
&\left.\cdot\left(\frac{1}{4 T^{2}} \dot{x}_{\mu}(t) \dot{x}_{\nu}\left(t^{\prime}\right)\right)\left(\psi_{1}^{\nu}+\kappa^{\nu}\left(t^{\prime}\right)\right)\right] \\
&-\int d t \frac{1}{2 T}\left[\left(e^{-i a} \bar{\psi}_{2}^{\mu}+\bar{\kappa}^{\mu}(t)\right) \eta_{\mu \nu}\left(\psi_{1}^{\nu}+\kappa^{\nu}(t)\right]\right\rangle,
\end{aligned}
$$

where the correlators are with respect to the action for the fluctuations. The $a$ integral can be done as follows:

$$
\begin{aligned}
\int_{0}^{2 \pi} \frac{d a}{2 \pi} e^{i k a+e^{-i a} \bar{\psi}_{2} \cdot \psi_{1}} & =\int_{0}^{2 \pi} \frac{d a}{2 \pi} e^{i k a} \sum_{n=0}^{4} \frac{1}{n !}\left(e^{-i a} \bar{\psi}_{2} \cdot \psi_{1}\right)^{n} \\
& = \begin{cases}\frac{1}{k !}\left(\bar{\psi}_{2} \cdot \psi_{1}\right)^{k}, & k \geq 0 \\
0, & k<0 .\end{cases}
\end{aligned}
$$

Thus (53) becomes, upon substituting $k=2$ and using $\theta(0)=$ $1 / 2$,

$$
\begin{aligned}
& \int d t d t^{\prime} \frac{1}{4 T^{2}} \bar{\psi}_{2} \cdot \psi_{1} \bar{\psi}_{2}^{\mu} \dot{x}_{\mu}(t) \dot{x}_{\nu}\left(t^{\prime}\right) \psi_{1}^{\nu}+\frac{1}{2} \\
& \cdot \int d t d t^{\prime}\left(\bar{\psi}_{2} \cdot \psi_{1}\right)^{2}\left(\frac{1}{4 T^{2}} \dot{x}_{\mu}(t) \dot{x}_{v}\left(t^{\prime}\right) \theta\left(t-t^{\prime}\right)\right) \eta^{\mu \nu} \\
& -\frac{1}{T} \int d t\left(\bar{\psi}_{2} \cdot \psi_{1}\right)^{2}
\end{aligned}
$$

since the one-point functions of $\kappa$ 's vanish. Now, we must evaluate the rest of the integrals. Let us first do the $x$ pathintegral. Again, $x$ 's can be separated into background and fluctuations

$$
x_{\mu}(t)=x_{\mu}(0)+\left(x_{\mu}(1)-x_{\mu}(0)\right) t+y_{\mu}(t),
$$

where $y(0)=y(1)=0$ so that

$$
\begin{aligned}
\dot{x}_{\mu}(t) \dot{x}_{\nu}\left(t^{\prime}\right)= & \left(x_{\mu}(1)-x_{\mu}(0)\right)\left(x_{\nu}(1)-x_{\nu}(0)\right) \\
& +\left(x_{\mu}(1)-x_{\mu}(0)\right) \dot{y}_{\nu}\left(t^{\prime}\right) \\
& +\left(x_{v}(1)-x_{v}(0)\right) \dot{y}_{\mu}(t) \\
& +\dot{y}_{\mu}(t) \dot{y}_{\nu}\left(t^{\prime}\right)
\end{aligned}
$$

and the $x$ action becomes

$$
\begin{aligned}
& \int d t \frac{1}{4 T}\left((x(1)-x(0))^{2}+2(x(1)-x(0)) \cdot \dot{y}+\dot{y}^{2}\right) \\
& =\int d t \frac{1}{4 T}\left((x(1)-x(0))^{2}+\dot{y}^{2}\right) .
\end{aligned}
$$

Thus the $\dot{x}$ correlator yields, upon substituting the values for $x(1)$ and $x(0)$ and again taking into account the contribution from the free integral over $y$ 's,

$$
\begin{gathered}
\left\langle\dot{x}_{\mu}(t) \dot{x}_{\nu}\left(t^{\prime}\right)\right\rangle=\frac{1}{(4 \pi T)^{2}}\left[\left(x_{\mu}^{\prime}-x_{\mu}\right)\left(x_{\nu}^{\prime}-x_{\nu}\right)\right. \\
\left.+\eta_{\mu \nu}\left(-2 T\left(1-\delta\left(t-t^{\prime}\right)\right)\right)\right] e^{-\left(x^{\prime}-x\right)^{2} / 4 T},
\end{gathered}
$$

where we have taken the $\dot{y}$ correlator from [36] and used the fact that one-point functions vanish. Thus doing the $t$ integral in (55), one gets, upon using $\int_{0}^{1} d t d t^{\prime}\left(1-\delta\left(t-t^{\prime}\right)\right)=0$, $\int_{0}^{1} d t d t^{\prime} \theta\left(t-t^{\prime}\right)=1 / 2$, and $\int_{0}^{1} d t d t^{\prime}\left(1-\delta\left(t-t^{\prime}\right)\right) \theta\left(t-t^{\prime}\right)=0$,

$$
\begin{aligned}
& \frac{1}{(4 \pi T)^{2}}\left[\frac { 1 } { 4 T ^ { 2 } } \left(\left(x_{\mu}^{\prime}-x_{\mu}\right)\left(x_{\nu}^{\prime}-x_{\nu}\right) \bar{\psi}_{2}^{\mu} \psi_{1}^{\nu} \bar{\psi}_{2} \cdot \psi_{1}\right.\right. \\
& \left.\left.+\frac{1}{4}\left(\bar{\psi}_{2} \cdot \psi_{1}\right)^{2}\left(x^{\prime}-x\right)^{2}\right)-\frac{1}{T}\left(\bar{\psi}_{2} \cdot \psi_{1}\right)^{2}\right] \\
& \cdot e^{-\left(x^{\prime}-x\right)^{2} / 4 T}
\end{aligned}
$$

which, upon doing the $T$ integral, reduces to

$$
=\left(\bar{\psi}_{2}^{\mu} \psi_{1}^{\nu} \bar{\psi}_{2} \cdot \psi_{1}\right) \partial_{\mu}^{\prime} \partial_{\nu}^{\prime} \frac{1}{4 \pi^{2}\left(x^{\prime}-x\right)^{2}}
$$

since

$$
\begin{aligned}
\int_{0}^{\infty} d T \frac{1}{(4 \pi T)^{2}} \frac{1}{T} e^{-\left(x^{\prime}-x\right)^{2} / 4 T} & =\frac{1}{\pi^{2}\left(x^{\prime}-x\right)^{4}}, \\
\int_{0}^{\infty} d T \frac{1}{(4 \pi T)^{2}} \frac{1}{4 T^{2}} e^{-\left(x^{\prime}-x\right)^{2} / 4 T} & =\frac{2}{\pi^{2}\left(x^{\prime}-x\right)^{6}} .
\end{aligned}
$$


We will now prove that (61) can be reexpressed as

$$
\int_{0}^{2 \pi} \frac{d a}{2 \pi}\left\langle\bar{\psi}_{2}\left|\hat{\bar{\psi}}^{\mu} \widehat{\psi}^{\nu}\left(e^{-i a\left(\hat{\bar{\psi}}^{\mu} \widehat{\psi}_{\mu}-2\right)}\right)\right| \psi_{1}\right\rangle \partial_{\nu}^{\prime} \partial_{\mu}^{\prime} \frac{1}{4 \pi^{2}\left(x^{\prime}-x\right)^{2}} .
$$

First of all the latter is equal, due to the property of coherent states, $\left\langle\bar{\psi}_{2}\right| \widehat{\psi}^{v}=\left(\partial / \partial \bar{\psi}_{2 v}\right)\left\langle\bar{\psi}_{2}\right|$, to

$$
\int_{0}^{2 \pi} \frac{d a}{2 \pi} \bar{\psi}_{2}^{\mu} \frac{\partial}{\partial \bar{\psi}_{2 v}}\left\langle\bar{\psi}_{2}\left|\left(e^{-i a\left(\hat{\bar{\psi}}^{\mu} \widehat{\psi}_{\mu}-2\right)}\right)\right| \psi_{1}\right\rangle \partial_{\nu}^{\prime} \partial_{\mu}^{\prime} \frac{1}{4 \pi^{2}\left(x^{\prime}-x\right)^{2}}
$$

which, from Appendix B, is

$$
\begin{gathered}
=\int_{0}^{2 \pi} \frac{d a}{2 \pi} \bar{\psi}_{2}^{\mu} \frac{\partial}{\partial \bar{\psi}_{2 v}} e^{e^{-i a} \bar{\psi}_{2} \cdot \psi_{1}+2 i a} \partial_{\nu}^{\prime} \partial_{\mu}^{\prime} \frac{1}{4 \pi^{2}\left(x^{\prime}-x\right)^{2}} \\
=\int_{0}^{2 \pi} \frac{d a}{2 \pi} \bar{\psi}_{2}^{\mu} \frac{\partial}{\partial \bar{\psi}_{2 \nu}} \sum_{n=0}^{4} \frac{1}{n !}\left(e^{-i a} \bar{\psi}_{2} \cdot \psi_{1}\right)^{n} e^{2 i a} \partial_{\nu}^{\prime} \partial_{\mu}^{\prime} \frac{1}{4 \pi^{2}\left(x^{\prime}-x\right)^{2}}
\end{gathered}
$$

from which only $n=2$ will survive the $a$ integration, leading to

$$
\begin{aligned}
& =\bar{\psi}_{2}^{\mu} \frac{\partial}{\partial \bar{\psi}_{2 v}} \frac{1}{2 !}\left(\bar{\psi}_{2} \cdot \psi_{1}\right)^{2} \partial_{\nu}^{\prime} \partial_{\mu}^{\prime} \frac{1}{4 \pi^{2}\left(x^{\prime}-x\right)^{2}} \\
& =\left(\bar{\psi}_{2}^{\mu} \psi_{1}^{\nu} \bar{\psi}_{2} \cdot \psi_{1}\right) \partial_{\mu}^{\prime} \partial_{\nu}^{\prime} \frac{1}{4 \pi^{2}\left(x^{\prime}-x\right)^{2}}
\end{aligned}
$$

which is the same as (61).

But (63) can also be written as ${ }^{2}$

$$
\begin{gathered}
\int_{0}^{2 \pi} \frac{d a}{2 \pi}\left\langle x^{\prime}, \bar{\psi}_{2}\left|\widehat{\bar{\psi}}^{\mu} p_{\mu} \widehat{\psi}^{\nu} p_{\nu} \frac{1}{p^{2}}\left(e^{-i a\left(\hat{\bar{\psi}}^{\mu} \widehat{\psi}_{\mu}-2\right)}\right)\right| x, \psi_{1}\right\rangle \\
=\left\langle x^{\prime}, \bar{\psi}_{2}\left|\widehat{\bar{\psi}}^{\mu} p_{\mu} \widehat{\psi}^{v} p_{v} \frac{1}{p^{2}} \delta_{\widehat{N}, 2}\right| x, \psi_{1}\right\rangle .
\end{gathered}
$$

Now again, this form could have been arrived at more directly by starting out with

$$
\int_{0}^{\infty} d T \int d \theta \int d \bar{\theta} \int d a\left\langle X^{\prime}\left|e^{-T H-i \theta \overline{\mathrm{Q}}-i \bar{\theta} \mathrm{Q}-i a(\widehat{N}-2)}\right| X\right\rangle .
$$

And again, as in the previous section, the usual path that one traverses is from the above expression to the path-integral form in (46). The above equation yields

$$
\begin{aligned}
& \int_{0}^{\infty} d T \int d \theta \int d \bar{\theta}\left\langle X^{\prime} ; \rho, \sigma\right| \\
& \cdot e^{-T H}[1-i \theta \bar{Q}-i \bar{\theta} Q-(\theta \bar{Q} \bar{\theta} Q)]|X ; \mu, \nu\rangle \\
& =-\int_{0}^{\infty} d T\left\langle X^{\prime} ; \rho, \sigma\left|e^{-T H}(\bar{Q} Q)\right| X ; \mu, \nu\right\rangle \\
& =-\left\langle X^{\prime} ; \rho, \sigma\left|\frac{(\bar{Q} Q)}{H}\right| X ; \mu, \nu\right\rangle,
\end{aligned}
$$

where

$$
|X ; \mu, \nu\rangle=|x\rangle \otimes \bar{\psi}^{\mu} \bar{\psi}^{v}|0\rangle
$$

and we have made a change of basis from that of the coherent states to the Maxwell one, which because of the delta function $\delta_{\widehat{N}, 2}$ arising from (70) upon integrating over $a$ is of the form

$$
\widehat{\bar{\psi}}^{\mu} \widehat{\bar{\psi}}^{v}|0\rangle=|\mu, v\rangle
$$

that is, the number of $\widehat{\bar{\psi}}$ operators is restricted to 2 . This gels well with the 2-form (gauge boson field strength) wavefunction which is

$$
F_{\mu \nu}(x) \widehat{\bar{\psi}}^{\mu} \widehat{\bar{\psi}}^{\nu}|0\rangle \text {. }
$$

In this basis, one has, from (72),

$$
-\left[\left\langlex^{\prime}\left|\otimes\langle 0| \widehat{\psi}^{\kappa} \widehat{\psi}^{\varphi}\right]\left[\frac{\hat{\bar{\psi}}^{\rho} p_{\rho} \widehat{\psi}^{\sigma} p_{\sigma}}{p^{2}}\right]\left[|x\rangle \otimes \widehat{\bar{\psi}}^{\mu} \widehat{\bar{\psi}}^{\nu}|0\rangle\right] .\right.\right.
$$

Notice that this is the same as (69) where the delta function $\delta_{\widehat{N}, 2}$ acts on the ket there to reduce it to the above form, and then since the operator in the middle contains $\hat{\bar{\psi}}^{\rho}$ and $\widehat{\psi}^{\sigma}$ the only bras which can have meaningful inner products are of the above form. We emphasize this because later we will see that, for the case of the left-handed spin-half particle in Section 5, the kets and bras will have different operator contents, since the operator in the middle will be of the form $\widehat{\psi}^{\mu} p_{\mu}$. Now,

$$
\begin{aligned}
& {\left[\hat{\bar{\psi}}^{\rho} p_{\rho} \widehat{\psi}^{\sigma} p_{\sigma}\right]\left[|x\rangle \otimes \widehat{\bar{\psi}}^{\mu} \widehat{\bar{\psi}}^{\nu}|0\rangle\right]} \\
& \quad=p_{\rho} p_{\sigma}|x\rangle \otimes\left[\eta^{\mu \sigma} \widehat{\bar{\psi}}^{\rho} \widehat{\bar{\psi}}^{\nu}-\eta^{\nu \sigma} \widehat{\bar{\psi}}^{\rho} \widehat{\bar{\psi}}^{\mu}\right]|0\rangle .
\end{aligned}
$$

Thus taking the overlap

$$
-\left\langle x^{\prime} ; \kappa, \varphi\left|\left(\hat{\bar{\psi}}^{\rho} p_{\rho} \widehat{\psi}^{\sigma} p_{\sigma}\right)\right| x ; \mu, \nu\right\rangle
$$

one gets

$$
\begin{aligned}
- & {\left[\left\langle x^{\prime}\right| \otimes\langle 0|\right]\left[\widehat{\psi}^{\kappa} \widehat{\psi}^{\varphi} p_{\rho} p_{\sigma}\left[\eta^{\mu \sigma} \widehat{\bar{\psi}}^{\rho} \widehat{\bar{\psi}}^{\nu}-\eta^{\nu \sigma} \hat{\bar{\psi}}^{\rho} \widehat{\bar{\psi}}^{\mu}\right]\right] } \\
& \cdot[|0\rangle \otimes|x\rangle] \\
= & -\left\langle x^{\prime}\right| p_{\rho} p_{\sigma}\left[\eta^{\mu \sigma}\left\{\eta^{\varphi \rho} \eta^{\kappa \nu}-\eta^{\varphi \nu} \eta^{\kappa \rho}\right\}\right. \\
& \left.-\eta^{\nu \sigma}\left\{\eta^{\varphi \rho} \eta^{\kappa \mu}-\eta^{\varphi \mu} \eta^{\kappa \rho}\right\}\right]|x\rangle \\
= & -\left\langle x^{\prime}\right|\left[p^{\varphi} p^{\mu} \eta^{\kappa \nu}-p^{\kappa} p^{\mu} \eta^{\varphi \nu}-p^{\varphi} p^{\nu} \eta^{\kappa \mu}+p^{\kappa} p^{\nu} \eta^{\varphi \mu}\right]
\end{aligned}
$$

$\cdot|x\rangle$

and hence the propagator that is obtained from (76) in this basis is

$$
\begin{aligned}
& -\left\langle x^{\prime}\right| \\
& \cdot \frac{\left[p^{\varphi} p^{\mu} \eta^{\kappa \nu}-p^{\kappa} p^{\mu} \eta^{\varphi \nu}-p^{\varphi} p^{\nu} \eta^{\kappa \mu}+p^{\kappa} p^{\nu} \eta^{\varphi \mu}\right]}{p^{2}}|x\rangle .
\end{aligned}
$$

But this is precisely the propagator

$$
\left\langle\partial^{\varphi} A^{\kappa} \partial^{\mu} A^{\nu}-\partial^{\kappa} A^{\varphi} \partial^{\mu} A^{\nu}-\partial^{\varphi} A^{\kappa} \partial^{\nu} A^{\mu}+\partial^{\kappa} A^{\varphi} \partial^{\nu} A^{\mu}\right\rangle
$$


or

$$
\left\langle F^{\varphi \kappa}\left(x^{\prime}\right) F^{\mu \nu}(x)\right\rangle
$$

if the propagator $\left\langle A^{\mu}\left(x^{\prime}\right) A^{\nu}(x)\right\rangle$ is

$$
\left\langle x^{\prime}\left|\frac{\eta^{\mu \nu}-(1-\xi)\left(p^{\mu} p^{\nu} / p^{2}\right)}{p^{2}}\right| x\right\rangle
$$

which is the gauge boson correlator in an arbitrary gauge; the freedom to choose $\xi$ follows from the symmetry of (81) under interchange of the Lorentz indices ${ }^{3}$. Thus the appropriate path-integral representation of the gauge boson (field strength) propagator in the coherent state basis is (46), which in the light of all this is the same as (69), which in turn gives rise to the above propagator, when one transforms to the Maxwell basis, via (76). The derivation here can be compared with that in [24] to see the advantages of this method.

\section{The Hamiltonian BRST Treatment}

In the above two derivations, we skirted the issue of ghosts that naturally arise when gauge-fixing a set of fields, and we will remedy the situation here by doing a full Hamiltonian BRST analysis. In particular, we will deal only with the spin1 case and that too with a different choice of the ChernSimmons parameter $s$ from the above section, which will lead us directly to the propagator in the Feynman-'t Hooft gauge. This is somewhat puzzling and we are yet to figure out a method that would yield (83) directly.

The action that we will take is the same as before and is given by

$$
\begin{aligned}
& \int_{0}^{1} d T\left[p^{\mu} \dot{x}_{\mu}+i \bar{\psi}^{\mu} \dot{\psi}_{\mu}-\frac{1}{2} e p^{\mu} p_{\mu}-i \beta \bar{\psi}^{\mu} p_{\mu}-i \bar{\beta} \psi^{\mu} p_{\mu}\right. \\
& \left.\quad-a \bar{\psi}^{\mu} \psi_{\mu}+s a\right],
\end{aligned}
$$

where $s$ is an integer that takes value 1, instead of zero as in the above section.

There are two bosonic gauge symmetries, $p^{\mu} p_{\mu}$ and $\bar{\psi}^{\mu} \psi_{\mu}$, and two fermionic ones, $\bar{\psi}^{\mu} p_{\mu}$ and $\psi^{\mu} p_{\mu}$, and for each of them we need to define ghost fields $\mathscr{C}^{A}=\left(\mathscr{C}, \mathscr{C}^{\prime}, \overline{\mathscr{C}}^{\prime}, \mathscr{C}^{\prime \prime}\right)$ and ghost momenta $\mathscr{P}_{A}=\left(\mathscr{P}, \mathscr{P}^{\prime}, \overline{\mathscr{P}}^{\prime}, \mathscr{P}^{\prime \prime}\right)$ such that $\left[\mathscr{P}_{A}, \mathscr{C}^{B}\right\}=-i \delta_{A}^{B}$. $\left(\mathscr{C}^{\prime}, \overline{\mathscr{C}}^{\prime}\right)$ are bosonic ghosts and correspond to $\bar{\psi}^{\mu} p_{\mu}$ and $\psi^{\mu} p_{\mu}$, respectively, while the rest are fermionic ghosts and correspond to $p^{\mu} p_{\mu}$ and $\bar{\psi}^{\mu} \psi_{\mu}$, respectively.

We will now adapt the treatment of [44] to the case at hand. The treatment there deals with particles of any spin, corresponding to which there are many more ghosts $\mathscr{C}_{i j}$ corresponding to the more generalized R-symmetry $\psi_{i}^{\mu} \psi_{\mu j}$ instead of just $\bar{\psi}^{\mu} \psi_{\mu}$ and hence a more complicated analysis. The starting action is

$$
\begin{aligned}
& \int d T\left[p^{\mu} \dot{x}_{\mu}+i \psi_{i}^{\mu} \dot{\psi}_{\mu i}-\frac{1}{2} e p^{\mu} p_{\mu}-i \beta_{i} \psi_{i}^{\mu} p_{\mu}\right. \\
& \left.-\frac{i}{2} a_{i j} \psi_{i}^{\mu} \psi_{j \mu}\right] .
\end{aligned}
$$

Firstly, the quantum BRST operator can be written as a graded sum

$$
\Omega=\sum_{p \geq 0} \Omega^{p}
$$

Then starting from

$$
\Omega^{0}=\mathscr{C}^{A} G_{A}=\mathscr{C} H+\mathscr{C}_{i} Q_{i}+\mathscr{C}_{i j} J_{i j}
$$

where $H=p^{2}, Q_{i}=\psi_{i} \cdot p$, and $J_{i j}=i \psi_{i} \cdot \psi_{j}$, and imposing the nilpotency of the BRST charge, one can recursively obtain the higher operators. Then the quantum gauge-fixed Hamiltonian operator can be written as

$$
H_{q u}=H_{\mathrm{BRST}}-i\{K, \Omega\},
$$

where the first term is the BRST-invariant Hamiltonian and $K$ a gauge fixing fermion, the latter being BRST-invariant for any choice of $K$ thanks to the nilpotency of $\Omega$. Now since $H$ itself enters as a constraint in (87) one can set $H_{\mathrm{BRST}}=0$ and thus have

$$
H_{q u}=-i\{K, \Omega\} .
$$

Then one can use the gauge fixing fermion

$$
K=-\widehat{E}^{A} \mathscr{P}_{A},
$$

where $\widehat{E}^{A}=\left(2 T, 0, \theta_{i j}\right)$ are the gauge fixations of $\left(e, \beta_{i}, a_{i j}\right)$ in the action in (85), where $\theta_{i j}$ is a $N \times N$ skew diagonal matrix, dependent on $N / 2=n$ angular variables $\theta_{k}$, with $k=1, \ldots, n . N / 2$ is the spin of the particle. Note that there is no term akin to $s a$ in (84) and it is only for $N=2$ that it manifests itself $[42,43]$. In any case with these choices for $\widehat{E}^{A}$, the Hamiltonian operator becomes

$$
H_{q u}=T p^{2}+\frac{1}{2} \theta_{i j} J_{i j}-\theta_{i j} \mathscr{C}_{i} \mathscr{P}_{j}-2 \theta_{i j} \mathscr{C}_{i m} \mathscr{P}_{j m}
$$

and, subsequently, the modified version of the above pathintegral can be rewritten in terms of these variables.

In the case at hand, since $\bar{\psi}_{\mu}$ and $\psi_{\mu}$ are related to $\psi_{\mu i}$ in the following manner

$$
\begin{aligned}
& \bar{\psi}_{\mu}=\frac{1}{\sqrt{2}}\left(\psi_{1 \mu}+i \psi_{2 \mu}\right), \\
& \psi_{\mu}=\frac{1}{\sqrt{2}}\left(\psi_{1 \mu}-i \psi_{2 \mu}\right)
\end{aligned}
$$

one has

$$
\begin{aligned}
\frac{1}{2} \theta_{i j} J_{i j} & =\frac{i}{2}\left(\theta_{12} \psi_{1} \cdot \psi_{2}+\theta_{21} \psi_{2} \cdot \psi_{1}\right) \\
& =-\frac{i \vartheta}{2}\left(\psi_{1} \cdot \psi_{2}-\psi_{2} \cdot \psi_{1}\right)=\vartheta \bar{\psi} \cdot \psi,
\end{aligned}
$$

where we have used the fact that, due to the skew diagonal nature of $\vartheta^{\prime}$ s, $\theta_{12}=-\theta_{21}=-\vartheta$. Also $\left(\mathscr{C}^{\prime}, \overline{\mathscr{C}}^{\prime}\right)$ are related to $\mathscr{C}_{i}$ via

$$
\begin{aligned}
& \mathscr{C}^{\prime}=\frac{1}{\sqrt{2}}\left(\mathscr{C}_{1}-i \mathscr{C}_{2}\right), \\
& \overline{\mathscr{C}}^{\prime}=\frac{1}{\sqrt{2}}\left(\mathscr{C}_{1}+i \mathscr{C}_{2}\right)
\end{aligned}
$$

so that 


$$
\begin{aligned}
\theta_{i j} \mathscr{C}_{i} \mathscr{P}_{j}= & -\vartheta\left(\mathscr{C}_{1} \mathscr{P}_{2}-\mathscr{C}_{2} \mathscr{P}_{1}\right)=-\frac{i}{2} \vartheta\left[\left(\mathscr{C}^{\prime}+\overline{\mathscr{C}}^{\prime}\right)\left(\mathscr{P}^{\prime}-\overline{\mathscr{P}}^{\prime}\right)-\left(\mathscr{C}^{\prime}-\overline{\mathscr{C}}^{\prime}\right)\left(\mathscr{P}^{\prime}+\overline{\mathscr{P}}^{\prime}\right)\right] \\
& =-i \vartheta\left(\overline{\mathscr{C}}^{\prime} \mathscr{P}^{\prime}-\mathscr{C}^{\prime} \overline{\mathscr{P}}^{\prime}\right)=\vartheta\left(\overline{\mathscr{C}}^{\prime} \frac{\partial}{\partial \overline{\mathscr{C}}^{\prime}}-\mathscr{C}^{\prime} \frac{\partial}{\partial \mathscr{C}^{\prime}}\right) .
\end{aligned}
$$

Thus this is the difference of the bosonic ghost number operators, multiplied by $\vartheta$ (we have used $\vartheta$ instead of $\theta$, in order to not confuse it with the gauge-fixed form of $\beta$ of previous sections, and in fact it is rather $a$ of the previous section). The last term $2 \theta_{i j} \mathscr{C}_{i m} \mathscr{P}_{i m}$ in (91) is equal to zero for $N=2$, because of the antisymmetry of the indices for each factor in the term. $H_{q u}$ thus becomes

$$
H_{q u}=T p^{\mu} p_{\mu}+\vartheta\left(\bar{\psi}^{\mu} \psi_{\mu}+N_{\overline{\mathscr{C}}^{\prime}}-N_{\mathscr{C}^{\prime}}\right)-s \vartheta,
$$

where we have incorporated the gauge-fixed form of $s a$ that is present in our case. The path-integral then becomes

$$
\begin{aligned}
& \int_{0}^{\infty} d T \int_{0}^{2 \pi} d \vartheta \int \mathscr{D} \mathscr{C} \int \mathscr{D} \mathscr{P} \int \mathscr{D} p \int_{x(0)=x}^{x(1)=x^{\prime}} \mathscr{D} x \\
& \cdot \int_{\psi(0)=\psi_{1}}^{\bar{\psi}(1)=\bar{\psi}_{2}} \mathscr{D} \psi \mathscr{D} \bar{\psi} e^{i \int_{0}^{1} d T\left[p^{\mu} \dot{x}_{\mu}+i \bar{\psi}^{\mu} \dot{\psi}_{\mu}+\dot{\mathscr{C}}^{A} \mathscr{P}_{A}-T p^{\mu} p_{\mu}-9\left(\bar{\psi}^{\mu} \psi_{\mu}+N_{\overline{\mathscr{C}}^{\prime}}-N_{\mathscr{G}^{\prime}}\right)+s \vartheta\right]+\bar{\psi}^{\mu} \psi_{\mu}(1)+i \mathscr{C}^{A} \mathscr{P}_{A}(1)} .
\end{aligned}
$$

Now, upon Wick rotation, where one also rotates $\mathscr{P}_{A} \rightarrow i \mathscr{P}_{A}$ and $\vartheta \rightarrow i \vartheta$ one gets

$$
\begin{aligned}
& \int_{0}^{\infty} d T \int_{0}^{2 \pi} d \mathscr{\vartheta} \int \mathscr{D} \mathscr{C} \int \mathscr{D} \mathscr{P} \int \mathscr{D} p \int_{x(0)=x}^{x(1)=x^{\prime}} \mathscr{D} x \\
& \cdot \int_{\psi(0)=\psi_{1}}^{\bar{\psi}(1)=\bar{\psi}_{2}} \mathscr{D} \psi \mathscr{D} \bar{\psi} e^{\int_{0}^{1} d T\left[i p^{\mu} \dot{x}_{\mu}-\bar{\psi}^{\mu} \dot{\psi}_{\mu}-\dot{\mathscr{C}}^{A} \mathscr{P}_{A}-T p^{\mu} p_{\mu}+i \vartheta\left(\bar{\psi}^{\mu} \psi_{\mu}+N_{\overline{\mathscr{G}}^{\prime}}-N_{\mathscr{C}^{\prime}}\right)-i s 9\right]+\bar{\psi}^{\mu} \psi_{\mu}(1)+\mathscr{C}^{A} \mathscr{P}_{A}(1)} .
\end{aligned}
$$

In general, one should also impose boundary conditions on the ghosts as well and a set of BRST-invariant boundary conditions is to set all the ghosts to zero at the boundaries [24]. Since the path-integrals for the ghosts all contribute unity (the contributions from the determinantal pieces are all powers of $\left(T_{1}-T_{0}\right)$ [24], which is 1 here, and the boundaryvalue dependent pieces vanish in the exponent again leading to 1 ), doing the above integral, one again gets (Appendix B)

$$
\int_{0}^{\infty} d T \int_{0}^{2 \pi} \frac{d \vartheta}{2 \pi} e^{e^{-i \vartheta} \bar{\psi}_{2} \cdot \psi_{1}-i(s-2) \vartheta}\left\langle x^{\prime}\left|e^{-T p^{2}}\right| x\right\rangle,
$$

where the extra $(-2)$ is again a normal ordering constant. The normal ordering constants from the other two number operators in (98) cancel each other out. Upon doing the $\vartheta$ integral one gets as before

$$
\left\langle x^{\prime}, \bar{\psi}_{2}\left|\frac{1}{p^{2}} \delta_{\widehat{N}, 1}\right| x, \psi_{1}\right\rangle
$$

upon setting $s=1$, which upon reverting to the index basis yields

$$
\begin{aligned}
\left\langle x^{\prime}\left|\otimes\left\langle 0\left|\widehat{\psi}_{\mu} \frac{1}{p^{2}} \hat{\bar{\psi}}_{\nu}\right| 0\right\rangle \otimes\right| x\right\rangle \\
=\left\langle x^{\prime}\left|\frac{\eta^{\mu \nu}}{p^{2}}\right| x\right\rangle .
\end{aligned}
$$

Thus as noted above, one gets the propagator in the Feynman-'t Hooft gauge, and it would be nice to figure out a method that gives (83) directly.

\section{Propagator for the $S O(n)$ Gauge Boson}

Let us now come to the case of the non-Abelian gauge boson for gauge group $S O(n)$. For it, on top of the ingredients necessary for spin, one has to incorporate those necessary for "color." Thus the action (45) has to be supplemented in the following fashion: 


$$
\begin{aligned}
L= & p^{\mu} \dot{x}_{\mu}+i \bar{\psi}^{\mu} \dot{\psi}_{\mu}+i \bar{\lambda}_{i} \dot{\lambda}_{i}-\frac{1}{2} e p^{\mu} p_{\mu}-i \beta \bar{\psi}^{\mu} p_{\mu} \\
& -i \bar{\beta} \psi^{\mu} p_{\mu}-a \bar{\psi}^{\mu} \psi_{\mu}-b \bar{\lambda}_{i} \lambda_{i}+\left(m-\frac{n}{2}\right) b,
\end{aligned}
$$

where $i$ runs over $n$ values and $m=2$. We have again set $s=0$ in a possible $s$ term since we want to project to the two-form sector. Of course we could also set $s$ to one, as in the previous section, in order to get the gauge boson propagator directly. Again, upon quantization, when $\lambda$ 's become operators, the number operator $\bar{\lambda}_{i} \lambda_{i}$ becomes ambiguous and has to be antisymmetrized, so that upon normal ordering it picks up a factor of $n / 2$. Thus choosing $m-n / 2$ essentially projects to the $m$-form sector in the internal space. In the path-integral, the only extra ingredient would come from the $\lambda$ and $b$ integrals and the entire previous integral (47) would get supplemented by (Appendix B)

$$
\begin{aligned}
& \int_{0}^{2 \pi} \frac{d b}{2 \pi} \\
& \cdot \int_{\lambda(0)=\lambda_{1}}^{\bar{\lambda}(1)=\bar{\lambda}_{2}} \mathscr{D} \lambda \mathscr{D} \bar{\lambda} e^{i \int_{0}^{1} d t\left(i \bar{\lambda}_{i} \dot{\lambda}_{i}-\bar{\lambda}_{i} b \lambda_{i}+b(m-(n / 2))\right)+\bar{\lambda}_{i} \lambda_{i}(1)} \\
& =\int_{0}^{2 \pi} \frac{d b}{2 \pi} e^{i m b} e^{e^{-i b} \bar{\lambda}_{i 2} \lambda_{i 1}} \\
& =\int_{0}^{2 \pi} \frac{d b}{2 \pi}\left\langle\bar{\lambda}_{i 2}\left|e^{-i b\left(\hat{\bar{\lambda}}_{i} \hat{\lambda}_{i}-m\right)}\right| \lambda_{i 1}\right\rangle=\left\langle\bar{\lambda}_{i 2}\left|\delta_{\widehat{N}, 2}\right| \lambda_{i 1}\right\rangle
\end{aligned}
$$

for $m=2$. The usual route in the literature is to proceed from the second term in the above equation to the pathintegral in (103) but as we have proven in Appendix B, with help from Appendix A, the first term in (104) follows directly from the path-integral. So for us Eq. (103) is the starting point, hence the inversion of the usual route. Again, since the delta function projects to states of the form $\hat{\bar{\lambda}}_{a} \hat{\bar{\lambda}}_{b}|0\rangle$, so that the full wave-function is now

$$
F_{\mu \nu a b}(x) \hat{\bar{\psi}}_{\mu} \hat{\bar{\psi}}_{\nu} \hat{\bar{\lambda}}_{a} \hat{\bar{\lambda}}_{b}|0\rangle,
$$

the entire expression for the gauge boson propagator gets supplemented by terms of the form, when one transforms from the coherent state basis to the gauge-index basis,

$$
\left\langle 0\left|\hat{\lambda}_{a} \hat{\lambda}_{c} \hat{\bar{\lambda}}_{b} \hat{\bar{\lambda}}_{d}\right| 0\right\rangle=-\left(\delta_{a b} \delta_{c d}-\delta_{a d} \delta_{c b}\right) .
$$

Hence the previous expression for the propagator simply gets augmented as follows:

$$
\begin{gathered}
-\left(\delta_{a b} \delta_{c d}-\delta_{a d} \delta_{c b}\right)\left(\partial_{\mu} \partial_{\nu} \eta_{\rho \sigma}-\partial_{\mu} \partial_{\sigma} \eta_{\rho \nu}-\mu \longleftrightarrow \rho\right) \\
\cdot \frac{1}{4 \pi^{2}\left(x^{\prime}-x\right)^{2}}=\left\langle F_{\mu \rho a c}\left(x^{\prime}\right) F_{\nu \sigma b d}(x)\right\rangle
\end{gathered}
$$

so that the gauge boson propagator is simply

$$
\begin{aligned}
& \left\langle A_{\mu a c}\left(x^{\prime}\right) A_{\nu b d}(x)\right\rangle=-\left\langle x^{\prime}\right| \\
& \cdot \frac{\eta^{\mu \nu}-(1-\xi)\left(p^{\mu} p^{\nu} / p^{2}\right)}{p^{2}}|x\rangle\left(\delta_{a b} \delta_{c d}-\delta_{a d} \delta_{c b}\right)
\end{aligned}
$$

which is the correct expression for the $S O(n)$ gauge boson propagator.

In order to get the $S U(n)$ propagator, one can consider $S O(2 n)$ first and then restrict it to its $S U(n)$ subgroup. Thus $S U(3)$ can be obtained by starting with $S O(6)$ and restricting it to its $S U(3)$ subgroup. $S U(2)$ on the other hand does not need this, as it is the double cover of $S O(3)$, and there is no need to restrict it to any subgroup. One would however need to take appropriate linear combinations of $S O(3) \lambda$ 's, in order to get the $S U(2)$ worldline fermions, with a concomitant change in the expression for the propagator. On the other hand if one wants to arrive at these gauge groups starting from some GUT gauge group, say $S O(10)$, it can be implemented by choosing $n=10$. However, this is not entirely satisfactory, since when considering SU (3) one wants to have solely $S U(3)$ gauge bosons propagating, and considering $S O(6)$ would be superfluous. For this, one has to then take recourse to the analysis in [31], wherein how to get the first quantized theory for any arbitrary mixed symmetry tensor multiplet, and in particular the adjoint representations, has been shown.

\section{Propagators for the Chiral Fermions of the Standard Model}

The strange hypercharge quantum numbers of the fermions of the Standard Model have natural geneses if one assumes that they stem from an $S U(5)$ or $S O(10)$ GUT. Thus the sixteen chiral fermions of a single generation can be broken up into sets that transform as totally antisymmetric tensors of rank $n$ of the $S U(5)$ subgroup of the $S O(10)$ gauge group. For the 16 of $S O(10), n$ would take values 0,2 , and 4 , corresponding to the $\mathbf{1}, \mathbf{1 0}$, and $\overline{\mathbf{5}}$ of $S U(5)$. We believe nature is telling us something important here and it might prove to be productive if one tries to explore the possible microscopic genesis of such a pattern. Let us first consider the fermions to be Dirac spinors. Choosing the above values of $n$ essentially restricts the states to

$$
\Psi_{i, i_{1} i_{2} \cdots i_{n}}(x) \hat{\bar{\lambda}}_{i_{1}} \hat{\bar{\lambda}}_{i_{2}} \cdots \hat{\bar{\lambda}}_{i_{n}}|0\rangle
$$

$\Psi_{\text {... }}(x)$ being the position space wave-function, which for $n=$ 0 forms the $\mathbf{1}$, for $n=2$, forms the $\mathbf{1 0}$, and for $n=4$ forms the $\overline{5}$ of $S U(5)[33,34,45]$. The beauty of it is that all the delicate hypercharge assignments come about precisely (see end of section), upon assuming

$$
Y=\frac{1}{2}\left(\hat{\bar{\lambda}}_{4} \hat{\lambda}_{4}+\hat{\bar{\lambda}}_{5} \hat{\lambda}_{5}\right)-\frac{1}{3}\left(\hat{\bar{\lambda}}_{1} \hat{\lambda}_{1}+\hat{\bar{\lambda}}_{2} \hat{\lambda}_{2}+\hat{\bar{\lambda}}_{3} \hat{\lambda}_{3}\right)
$$

where $\hat{\lambda}_{i}$, for $i=1$ to 3 , encode color while the rest encode flavor. In fact, this would precisely be the oscillator basis representation of a diagonal generator of the $S U(5)$ group, another tantalizing hint that this group might have to do something with nature.

The first index $i$ in (109) is the Dirac index and is associated with the states in (7), which we have suppressed for simplicity. The relevant action would have to be supplemented as follows: 


$$
\begin{aligned}
L= & p^{\mu} \dot{x}_{\mu}+i \bar{\psi}_{j} \dot{\psi}_{j}+i \bar{\lambda}_{i} \dot{\lambda}_{i}-\frac{1}{2} e p^{\mu} p_{\mu}+i \beta \psi^{\mu} p_{\mu} \\
& -a \bar{\lambda}_{i} \lambda_{i}+\left(n-\frac{5}{2}\right) a,
\end{aligned}
$$

where $\lambda$ 's are worldline fermions for internal symmetries and $i$ runs over 1 to 5 . $a$ is an auxiliary field that imposes the restriction that the rank of the tensor be $n$. The relevant pathintegral would be

$$
\begin{aligned}
& \int_{x_{\mu}(0)=x_{\mu}}^{x_{\mu}(1)=x_{\mu}^{\prime}} \mathscr{D} \int_{\psi_{i}(0)=\psi_{1 i}}^{\bar{\psi}_{i}(1)=\bar{\psi}_{2 i}} \mathscr{D} \psi \mathscr{D} \bar{\psi} \int_{\lambda_{k}(0)=\lambda_{k 1}}^{\bar{\lambda}_{k}(1)=\bar{\lambda}_{k 2}} \mathscr{D} \lambda \mathscr{D} \bar{\lambda} \int \mathscr{D} a \\
& \quad \times \int \frac{\mathscr{D} \beta \mathscr{D} e}{\operatorname{Vol}(\mathrm{Sym})} \mathscr{D} p e^{\int_{0}^{1} d t\left(i p^{\mu} \dot{x}_{\mu}-\bar{\psi}_{j} \dot{\psi}_{j}-\bar{\lambda}_{i} \dot{\lambda}_{i}-(1 / 2) e p^{\mu} p_{\mu}-i \beta \psi^{\mu} p_{\mu}-i a \bar{\lambda}_{i} \lambda_{i}+i(n-5 / 2) a\right)+\bar{\lambda}_{i} \lambda_{i}(1)+\bar{\psi}_{i} \psi_{i}(1)} .
\end{aligned}
$$

As before, the result of the $\lambda$ and $a$ path-integrals is

$$
\int \frac{d a}{(2 \pi)} e^{i n a} e^{e^{-i a} \bar{\lambda}_{k 2} \lambda_{k 1}}=\left\langle\bar{\lambda}_{i 2}\left|\delta_{\widehat{N}, n}\right| \lambda_{i 1}\right\rangle
$$

which, upon reverting from the coherent state basis to that in (109) (since the delta function restricts it to states of that form only), yields

$$
\left\langle 0\left|\widehat{\lambda}_{i_{1}} \hat{\lambda}_{i_{2}} \cdots \widehat{\lambda}_{i_{n}} \widehat{\bar{\lambda}}_{j_{1}} \widehat{\bar{\lambda}}_{j_{2}} \cdots \widehat{\bar{\lambda}}_{j_{n}}\right| 0\right\rangle=\delta_{j_{1} j_{2} \cdots j_{n}}^{\left[i_{1} i_{2} \cdots i_{n}\right]}
$$

which in turn yields the following form of the propagator:

$$
\frac{i}{\sqrt{2}}\left\langle x^{\prime}, i\left|\frac{1}{\gamma \cdot p}\right| x, j\right\rangle \delta_{j_{1} j_{2} \cdots j_{n}}^{\left[i_{1} i_{2} \cdots i_{n}\right]}
$$

where $\left[i_{1} i_{2} \cdots i_{n}\right]$ denotes complete antisymmetrization with respect to the indices, which is the correct expression for

$$
\left\langle\Psi_{i}^{i_{1} i_{2} \cdots i_{n}}\left(x^{\prime}\right) \bar{\Psi}_{j, j_{1} j_{2} \cdots j_{n}}(x)\right\rangle
$$

Now if one considers that the SM fermions consist only of lefthanded ones, then one has to factor in a term that effectively projects to the left-handed states. From (7), it is evident that if one imposes that the number of $\widehat{\bar{\psi}}_{i}$ operators be one, one is done. Thus the action has to be modified as follows:

$$
\begin{aligned}
& p^{\mu} \dot{x}_{\mu}+i \bar{\psi}_{j} \dot{\psi}_{j}+i \bar{\lambda}_{i} \dot{\lambda}_{i}-\frac{1}{2} e p^{\mu} p_{\mu}-i \beta \psi^{\mu} p_{\mu}-a \bar{\lambda}_{i} \lambda_{i} \\
& \quad+\left(n-\frac{5}{2}\right) a-b \bar{\psi}_{j} \psi_{j}
\end{aligned}
$$

where $b$ is the auxiliary field as before that imposes the said condition. In this case the normal ordering constant is 1 since $j$ runs over 2 values, and hence, in the absence of any ChernSimmons-like pure $b$ term, the projection is to the $\widehat{N}_{\bar{\psi}}=1$ sector. Using the same strategy as in Sections 2 and 3, it can easily be seen that, upon integrating over $\bar{\psi}$ 's and $x$ 's, the pathintegral corresponding to the above Lagrangian would finally boil down to (sans the $\lambda$ part)

$$
i\left\langle x^{\prime} \bar{\psi}_{2}\left|\widehat{\psi}^{\mu} p_{\mu} \frac{1}{p^{2}} \delta_{\widehat{N}, 1}\right| x, \psi_{1}\right\rangle
$$

and as before, transforming to the Dirac basis, one essentially gets either

$$
i\left[\left\langlex^{\prime}\left|\otimes\langle 0| \widehat{\psi}_{2} \widehat{\psi}_{1}\right]\left[\frac{\widehat{\psi}^{\mu} p_{\mu}}{p^{2}}\right]\left[|x\rangle \otimes \widehat{\bar{\psi}}_{j}|0\rangle\right]\right.\right.
$$

or

$$
i\left[\left\langlex^{\prime}|\otimes\langle 0|]\left[\frac{\widehat{\psi}^{\mu} p_{\mu}}{p^{2}}\right]\left[|x\rangle \otimes \widehat{\bar{\psi}}_{j}|0\rangle\right]\right.\right.
$$

as the surviving inner products, since the delta function essentially projects the ket to the $N=1$ sector, which is the sector of the left-handed states, and the operator in the middle has only a single $\widehat{\psi}^{\mu}$ which mandates that the bras are either twofold in $\widehat{\psi}_{i}$ operator content or zero, implying that they are right-handed states (cf. (7)). These can be combined and generated in terms of left-handed states by inserting $\widehat{\psi}^{0}$, taking the form

$$
i\left[\left\langlex^{\prime}\left|\otimes\langle 0| \widehat{\psi}_{i}\right]\left[\widehat{\psi}^{0} \frac{\widehat{\psi}^{\mu} p_{\mu}}{p^{2}}\right]\left[|x\rangle \otimes \widehat{\bar{\psi}}_{j}|0\rangle\right]\right.\right.
$$

which can easily be verified by using (7). Since this is the same as

$$
\frac{i}{2}\left\langle x^{\prime}, i\left|\frac{\gamma_{0} \gamma^{\mu} p_{\mu}}{p^{2}}\right| x, j\right\rangle
$$

where both the states are left-handed, one can write this as

$$
\frac{i}{2}\left\langle x^{\prime}, i\left|\frac{\sigma^{\mu} p_{\mu}}{p^{2}}\right| x, j\right\rangle
$$

which is the propagator for Weyl fermions. Upon augmenting with the internal symmetry part as in (115), it has the form

$$
\frac{i}{2}\left\langle x^{\prime}, i\left|\frac{\sigma^{\mu} p_{\mu}}{p^{2}}\right| x, j\right\rangle \delta_{j_{1} j_{2} \cdots j_{n}}^{\left[i_{1} i_{2} \cdots i_{n}\right]} .
$$

This thus has the following path-integral representation in the coherent state basis: 


$$
\begin{aligned}
& \int_{x_{\mu}(0)=x_{\mu}}^{x_{\mu}(1)=x_{\mu}^{\prime}} \mathscr{D} x \int_{\psi_{i}(0)=\psi_{1 i}}^{\bar{\psi}_{i}(1)=\bar{\psi}_{2 i}} \mathscr{D} \psi \mathscr{D} \bar{\psi} \int_{\lambda_{k}(0)=\lambda_{k 1}}^{\bar{\lambda}_{k}(1)=\bar{\lambda}_{k 2}} \mathscr{D} \lambda \mathscr{D} \bar{\lambda} \int \mathscr{D} a \int \mathscr{D} b \\
& \quad \times \int \frac{\mathscr{D} \beta \mathscr{D} e}{\operatorname{Vol}(\mathrm{Sym})} \mathscr{D} p e^{\int_{0}^{1} d t\left(i p^{\mu} \dot{x}_{\mu}-\bar{\psi}_{j} \dot{\psi}_{j}-\bar{\lambda}_{i} \dot{\lambda}_{i}-(1 / 2) e p^{\mu} p_{\mu}+i \beta \psi^{\mu} p_{\mu}-i a \bar{\lambda}_{i} \lambda_{i}-i b \bar{\psi}_{i} \psi_{i}+i(n-5 / 2) a\right)+\bar{\lambda}_{i} \lambda_{i}(1)+\bar{\psi}_{i} \psi_{i}(1)} .
\end{aligned}
$$

which differs from (112) in the $-i b \bar{\psi}_{i} \psi_{i}$ term.

For completeness we give the representations of the SM fermions in Table 1, where $\chi_{1}$ and $\chi_{2}$ stand for $\lambda_{4}$ and $\lambda_{5}$ above.

We have written them in terms of bras to avoid putting bars over all the creation operators. We have also made explicit only the first component of the Weyl fermions, hence the index 1 on all of them, and the second components can be obtained simply by replacing $\widehat{\psi}_{1}$ with $\widehat{\psi}_{2}$. Factors of $1 / 2$ associated with each $\epsilon$ tensor are assumed to be implicit. The states are written in terms of direct products of the bosonic and fermionic parts, with the bosonic parts forming the position space wave-functions upon conjugating them with $\langle x|$ 's. Thus, it is evident that all these states are the result of a set of fermionic creation operators acting on the vacuum and are superpartners of each other in the sense of ordinary spacetime supersymmetry. Akin to the fields in the latter, which can be expanded in terms of the Grassmann parameters $\theta_{i}$ belonging to a superfield $\Psi\left(x_{\mu}, \theta_{i}\right)$, the fermionic multiplets here too form a supermultiplet that results from expanding $\Phi\left(x_{\mu}, \bar{\psi}_{j}, \bar{\lambda}_{i}\right)$ and retaining terms even in $\bar{\lambda}$ and odd in $\bar{\psi}$. In a different way of looking at it, $\Phi\left(x_{\mu}, \bar{\psi}_{j}, \bar{\lambda}_{i}\right)$ can be understood to arise as follows:

$$
\begin{aligned}
& \Phi\left(x_{\mu}, \bar{\psi}_{j}, \bar{\lambda}_{i}\right) \\
& \quad=\left\langle x, \bar{\psi}, \bar{\lambda}\left|\sum_{n} \Psi_{i_{i} i_{1} i_{2} \cdots i_{n}}(x) \hat{\bar{\lambda}}_{i_{1}} \widehat{\bar{\lambda}}_{i_{2}} \cdots \widehat{\bar{\lambda}}_{i_{n}} \widehat{\bar{\psi}}_{i}\right| 0\right\rangle,
\end{aligned}
$$

where the different $\Psi$ 's, which are the wave-functions of fermions of the SM, are seen to belong in a single supermultiplet $\Phi$.

Let us now come to the issue of the various charges of the SM. The hypercharge operator is

$$
Y=-\frac{1}{3} \widehat{\bar{\lambda}}_{i} \widehat{\lambda}_{i}+\frac{1}{2} \widehat{\bar{\chi}}_{i} \widehat{\chi}_{i}
$$

and the isospin operator is

$$
T_{3 L}=\frac{1}{2}\left(\widehat{\bar{\chi}}_{1} \widehat{\chi}_{1}-\widehat{\bar{\chi}}_{2} \widehat{\chi}_{2}\right) .
$$

Hence the charge operator is

$$
Q=Y+T_{3 L}=-\frac{1}{3} \widehat{\bar{\lambda}}_{i} \hat{\lambda}_{i}+\widehat{\bar{\chi}}_{1} \widehat{\chi}_{1}
$$

Also,

$$
B-L=1-\frac{2}{3} \hat{\bar{\lambda}}_{i} \hat{\lambda}_{i}
$$

It is straightforward to check that all the charge and hypercharge assignments for the chiral generations are correct and, as we said, can be regarded as an explanation of the strange hypercharge assignments and the fractional charge assignments in the Standard Model. The way these computations are done is by noting that each of the bilinears above is actually a number operator for the different species of worldline fermions. So, for instance,

$$
Y=-\frac{1}{3} \sum_{i} N_{\bar{\lambda}_{i}}+\frac{1}{2} \sum_{i} N_{\bar{\chi}_{i}}
$$

and hence the action on say $\hat{\bar{\lambda}}_{m} \widehat{\bar{\chi}}_{1} \widehat{\bar{\psi}}_{1}|0\rangle \otimes\left|u_{m, L, 1}\right\rangle$ would give $-(1 / 3)(1)+(1 / 2)(1)=1 / 6$. In a similar spirit, the rest of them can be easily computed to be

$$
\begin{aligned}
& (B-L)_{v_{e L}^{c}}=1 \text {, } \\
& (B-L)_{v_{e L}}=-1, \\
& (B-L)_{e_{L}^{+}}=1, \\
& (B-L)_{e_{L}^{-}}=-1 \text {, } \\
& (B-L)_{u_{L \alpha}^{c}}=-\frac{1}{3}, \\
& (B-L)_{u_{L}^{\alpha}}=\frac{1}{3}, \\
& (B-L)_{d_{L \alpha}^{c}}=-\frac{1}{3} \text {, } \\
& (B-L)_{d_{L}^{\alpha}}=\frac{1}{3}, \\
& \left(T_{3 L}\right)_{v_{e L}^{c}}=0 \text {, } \\
& \left(T_{3 L}\right)_{v_{e L}}=\frac{1}{2} \text {, } \\
& \left(T_{3 L}\right)_{e_{L}^{+}}=0 \text {, } \\
& \left(T_{3 L}\right)_{e_{L}^{-}}=-\frac{1}{2}, \\
& \left(T_{3 L}\right)_{u_{L \alpha}^{c}}=0 \text {, } \\
& \left(T_{3 L}\right)_{u_{L}^{\alpha}}=\frac{1}{2}, \\
& \left(T_{3 L}\right)_{d_{L \alpha}^{c}}=0,
\end{aligned}
$$


TABLE 1: Fermionic states of the 1st chiral generation of the SM. In our indication of the SU(5) multiplet to which each state belongs we use the notation $\mathbf{n}_{Y}$ where $n$ is the dimension of the $S U(5)$ representation in which the state transforms and $Y$ is the $(S U(5)) U(1)_{Y}$ hypercharge.

\begin{tabular}{lr}
\hline$\langle 0| \widehat{\psi}_{1} \otimes\left\langle v_{L, 1}^{c}\right| \subset \mathbf{1}_{0}$ & $\langle 0| \widehat{\psi}_{1} \widehat{\chi}_{1} \widehat{\lambda}_{1} \widehat{\lambda}_{2} \hat{\lambda}_{3} \otimes\left\langle v_{L, 1}\right| \subset \overline{\mathbf{5}}_{-1 / 2}$ \\
$\langle 0| \widehat{\psi}_{1} \widehat{\chi}_{1} \widehat{\chi}_{2} \otimes\left\langle e_{L, 1}^{c}\right| \subset \mathbf{1 0}_{1}$ & $\langle 0| \widehat{\psi}_{1} \widehat{\chi}_{2} \widehat{\lambda}_{1} \widehat{\lambda}_{2} \widehat{\lambda}_{3} \otimes\left\langle e_{L, 1}\right| \subset \overline{\mathbf{5}}_{-1 / 2}$ \\
$\langle 0| \widehat{\psi}_{1} \epsilon_{m i j} \widehat{\lambda}_{i} \hat{\lambda}_{j} \otimes\left\langle u_{m, L, 1}^{c}\right| \subset \mathbf{1 0}_{-2 / 3}$ & $\langle 0| \widehat{\psi}_{1} \widehat{\chi}_{1} \widehat{\lambda}_{m} \otimes\left\langle u_{m, L, 1}\right| \subset \mathbf{1 0}_{1 / 6}$ \\
$\langle 0| \widehat{\psi}_{1} \bar{\chi}_{1} \widehat{\chi}_{2} \epsilon_{m i j} \widehat{\lambda}_{i} \widehat{\lambda}_{j} \otimes\left\langle d_{m, L, 1}^{c}\right| \subset \overline{\mathbf{5}}_{1 / 3}$ & $\langle 0| \widehat{\psi}_{1} \widehat{\chi}_{2} \widehat{\lambda}_{m} \otimes\left\langle d_{m, L, 1}\right| \subset \mathbf{1 0}_{1 / 6}$ \\
\hline
\end{tabular}

$$
\begin{array}{ll}
\left(T_{3 L}\right)_{d_{L}^{\alpha}}=-\frac{1}{2}, & \begin{array}{r}
\text { 7. The Massive Fermion Propagator in a Gauge } \\
\text { Field Background }
\end{array} \\
(Y)_{\gamma_{e L}^{c}}=0, & \text { Let us now extend our methods to the case of the dressed }
\end{array}
$$

\section{The Massive Fermion Propagator in a Gauge Field Background}$$
(Y)_{v_{e L}}=-\frac{1}{2}
$$$$
(Y)_{e_{L}^{+}}=1 \text {, }
$$$$
(Y)_{e_{L}^{-}}=-\frac{1}{2},
$$$$
(Y)_{u_{L \alpha}^{c}}=-\frac{2}{3},
$$$$
(Y)_{u_{L}^{\alpha}}=\frac{1}{6},
$$$$
(Y)_{d_{L \alpha}^{c}}=\frac{1}{3},
$$$$
(Y)_{d_{L}^{\alpha}}=\frac{1}{6},
$$$$
(Q)_{\gamma_{e L}^{c}}=0 \text {, }
$$$$
(Q)_{v_{e L}}=0 \text {, }
$$$$
(Q) e_{L}^{+}=1 \text {, }
$$$$
(Q)_{e_{L}^{-}}=-1 \text {, }
$$$$
(Q)_{u_{L \alpha}^{c}}=-\frac{2}{3},
$$$$
(Q)_{u_{L}^{\alpha}}=\frac{2}{3},
$$$$
(Q)_{d_{L \alpha}^{c}}=\frac{1}{3} \text {, }
$$$$
(Q)_{d_{L}^{\alpha}}=-\frac{1}{3} \text {. }
$$

Let us now extend our methods to the case of the dressed and massive fermion propagator. This is given by simply incorporating masses and backgrounds in (43):

$$
\begin{aligned}
& \frac{i}{\sqrt{2}}\left\langle x^{\prime}, \bar{\psi}_{2}, \bar{\lambda}_{2}\right| \\
& \cdot \frac{1}{\left(\gamma \cdot\left(p-g \mathbf{A}^{i j} \hat{\bar{\lambda}}_{i} \hat{\lambda}_{j}\right)-m\right)}\left|x, \psi_{1}, \lambda_{1}\right\rangle,
\end{aligned}
$$

where $\gamma$ has the same meaning as before, when acting on coherent states and $\mathbf{A}_{\mu}^{i j}=\left(A_{\mu}^{a} T^{a}\right)^{i j}$. Again, this can be written as

$$
\begin{aligned}
& \frac{i}{\sqrt{2}}\left\langle x^{\prime}, \bar{\psi}_{2}, \bar{\lambda}_{2}\right| \\
& \cdot \frac{\gamma \cdot\left(p-g \mathbf{A}^{i j} \hat{\bar{\lambda}}_{i} \hat{\lambda}_{j}\right)+m}{\left(\left(p-g \mathbf{A}^{i j} \hat{\bar{\lambda}}_{i} \hat{\lambda}_{j}\right)^{2}-\frac{1}{2} g \sigma^{\mu \nu} \mathbf{F}_{\mu \nu}^{i j} \hat{\bar{\lambda}}_{i} \hat{\lambda}_{j}-m^{2}\right)} \mid x, \psi_{1}, \\
& \left.\lambda_{1}\right\rangle,
\end{aligned}
$$

where $\sigma_{\mu \nu}=(i / 2)\left[\gamma_{\mu}, \gamma_{\nu}\right]$ and $\mathbf{F}_{\mu \nu}$ is the full non-Abelian gauge boson. First of all there is no need to introduce any $\psi_{5}$ in the worldline representation as is usual for the case of the massive fermion, since the identity that multiplies the mass in the above expression is identity in the Dirac index basis as well and would automatically place the mass in the right place in the numerator when the transformation to that basis is done. One can then call the numerator $Q$ and the denominator $H$ and as before exponentiate both (cf. (41))

$$
\int d T \int d \theta\left\langle X^{\prime}, \bar{\lambda}_{2}\left|e^{-T H-i \theta Q}\right| X, \lambda_{1}\right\rangle .
$$

This can be cast in the (Wick-rotated) path-integral form (for a fermion that is an $S U(n)$ tensor of rank $p$, cf. (125))

$$
\begin{aligned}
& \int_{x_{\mu}(0)=x_{\mu}}^{x_{\mu}(1)=x_{\mu}^{\prime}} \mathscr{D} x \int_{\psi_{i}(0)=\psi_{1 i}}^{\bar{\psi}_{i}(1)=\bar{\psi}_{2 i}} \mathscr{D} \psi \mathscr{D} \bar{\psi} \int_{\lambda_{k}(0)=\lambda_{k 1}}^{\bar{\lambda}_{k}(1)=\bar{\lambda}_{k 2}} \mathscr{D} \lambda \mathscr{D} \bar{\lambda} \int \mathscr{D} a \\
& \quad \cdot \int \frac{\mathscr{D} \beta \mathscr{D} e}{\operatorname{Vol}(\operatorname{Sym})} \mathscr{D} p \exp \left[\int_{0}^{1} d t\left(i p^{\mu} \dot{x}_{\mu}-\bar{\psi}_{j} \dot{\psi}_{j}-\bar{\lambda}_{i} \dot{\lambda}_{i}-\frac{1}{2} e\left(\pi^{\mu} \pi_{\mu}-m^{2}\right)-i \beta\left(\sqrt{2} \psi^{\mu} \pi_{\mu}+m\right)+\frac{i}{2} e g \psi^{\mu} \mathbf{F}_{\mu \nu}^{i j} \bar{\lambda}_{i} \lambda_{j} \psi^{\nu}-i a \bar{\lambda}_{i} \lambda_{i}+i\left(p-\frac{n}{2}\right) a\right)\right. \\
& \left.\quad+\bar{\lambda}_{i} \lambda_{i}(1)+\bar{\psi}_{j} \psi_{j}(1)\right],
\end{aligned}
$$


where $\pi_{\mu}=p_{\mu}-g \mathbf{A}_{\mu}^{i j} \bar{\lambda}_{i} \lambda_{j}$. See [13, 19, 21-23] for the pioneering treatment of the dressed propagator for the Abelian case and [12] for the non-Abelian one, the details here being somewhat akin to the latter reference, modulo the projection to the $S U(n)$ tensor and the explicit use of the formalism made in the next section.

The point might be raised that the $\beta$ term breaks supersymmetry since there is no $\psi_{5}$, but as long as no use is made of supersymmetry, it is ok to work without a $\psi_{5}$. Also the entire approach's vindication lies in the analysis in the next section, which gives the right form for the QED vertex, when restricted to the Abelian case and considering dressing by a single photon. Upon absorbing the factor of $\sqrt{2}$ into $\beta$ and calling $m / \sqrt{2} m^{\prime}$ (only in the term multiplying $\beta$ ) and integrating out the $p$, one ends up with

$$
\begin{aligned}
& \int_{x_{\mu}(0)=x_{\mu}}^{x_{\mu}(1)=x_{\mu}^{\prime}} \mathscr{D} \int_{\psi_{i}(0)=\psi_{1 i}}^{\bar{\psi}_{i}(1)=\bar{\psi}_{2 i}} \mathscr{D} \psi \mathscr{D} \bar{\psi} \int_{\lambda_{k}(0)=\lambda_{k 1}}^{\bar{\lambda}_{k}(1)=\bar{\lambda}_{k 2}} \mathscr{D} \lambda \mathscr{D} \bar{\lambda} \mathscr{D a} \\
& \quad \cdot \int \frac{\mathscr{D} \beta \mathscr{D} e}{\operatorname{Vol}(\operatorname{Sym})} \exp \left[\int_{0}^{1} d t\left(-\frac{1}{2 e}\left(\dot{x}_{\mu}-\beta \psi_{\mu}\right)^{2}-i \beta m^{\prime}+\frac{1}{2} e m^{2}-\bar{\psi}_{j} \dot{\psi}_{j}-\bar{\lambda}_{i} \dot{\lambda}_{i}+i g \dot{x}^{\mu} \mathbf{A}_{\mu}^{i j} \bar{\lambda}_{i} \lambda_{j}+\frac{i}{2} e g \psi^{\mu} \mathbf{F}_{\mu \nu}^{i j} \bar{\lambda}_{i} \lambda_{j} \psi^{\nu}-i a \bar{\lambda}_{i} \lambda_{i}+i\left(p-\frac{n}{2}\right) a\right)\right. \\
& \left.\quad+\bar{\lambda}_{i} \lambda_{i}(1)+\bar{\psi}_{j} \psi_{j}(1)\right]
\end{aligned}
$$

as the worldline representation of the massive dressed fermionic propagator.

\section{Applications to QED}

Here we derive the QED vertex using the worldline formalism developed in the previous section, adapted to the case of an Abelian gauge boson. It has to be formulated, like the propagator, on the open line, since one has an incoming and an outgoing propagator, with one photon insertion. One has to take the Fourier transform of the resultant expression with respect to the propagator endpoints to arrive at the momentum space expression. Since the path-integral that we start with, (156), corresponds to the propagator in position space with photon insertions (once $A_{\mu}$ are converted to plane waves), the momentum space expression in general would involve external leg factors which have to be amputated in order to arrive at the expression for the vertex.

Let us first work with the worldline expression for the scalar case since it would be present as a part of the spinor case. It has its genesis in the Fourier transform of the expression below

$$
\begin{aligned}
& \int_{x_{\mu}(0)=x_{\mu}^{\prime}}^{x_{\mu}(1)=x_{\mu}} \mathscr{D} x \\
& \quad \cdot \int \frac{\mathscr{D} e}{\operatorname{Vol}(\operatorname{Sym})} \exp \left[\int_{0}^{1} d t\left(-\frac{1}{2 e}\left(\dot{x}_{\mu}\right)^{2}+\frac{1}{2} e m^{2}+i g \dot{x}^{\mu} A_{\mu}\right)\right] .
\end{aligned}
$$

The external $A$ legs are to be represented by the plane-wave expansion of the $A$, leading to vertex operators. Explicitly, one has

$$
i g \dot{x}^{\mu} A_{\mu}=i g[\varepsilon \cdot \dot{x}(t)] e^{i k \cdot x(t)}
$$

This can be evaluated by exponentiating the $\varepsilon$ part and the resultant expression for an $n$-photon amplitude can be written as $\left\langle e^{\int d t J(t) \cdot x(t)}\right\rangle$ with

$$
J_{\mu}(t)=\sum_{i=1}^{n}\left(i k_{i \mu}+\varepsilon_{i \mu} \partial_{t_{i}}\right) \delta\left(t-t_{i}\right)
$$

It is given by (upon fixing $e$ to $2 T$ )

$$
\int_{0}^{\infty} d T \int_{x_{\mu}(0)=x_{\mu}^{\prime}}^{x_{\mu}(1)=x_{\mu}} \mathscr{D} x e^{\int_{0}^{1} d t\left(-(1 / 4 T) \dot{x}^{2}+m^{2} T+J \cdot x\right)}
$$

We first separate $x$ into background and quantum parts as follows:

$$
x_{\mu}(t)=x_{\mu}^{\prime}+\left(x_{\mu}-x_{\mu}^{\prime}\right) t+y_{\mu}(t)
$$

so that the above expression takes the form

$$
\begin{aligned}
& \int_{0}^{\infty} d T e^{m^{2} T} \\
& \cdot \int_{y_{\mu}(0)=0}^{y_{\mu}(1)=0} \mathscr{D} y e^{\int_{0}^{1} d t\left[\left(-(1 / 4 T)\left(\dot{y}^{2}+\left(x-x^{\prime}\right)^{2}\right)+J(t) \cdot\left(x+\left(x-x^{\prime}\right) t+y(t)\right)\right]\right.} .
\end{aligned}
$$

Integrating out $y$, one gets

$$
\int_{0}^{\infty} d T \frac{e^{m^{2} T}}{(4 \pi T)^{2}} e^{\int_{0}^{1} d t\left(-(1 / 4 T)\left(x-x^{\prime}\right)^{2}+J(t) \cdot\left(x+\left(x-x^{\prime}\right) t\right)\right)-T \int_{0}^{1} d t d t^{\prime}\left(J^{\mu}(t) \eta_{\mu \nu} \Delta\left(t, t^{\prime}\right) J^{\nu}\left(t^{\prime}\right)\right)}
$$


In the above equation we see that since we have $e^{m^{2} T}$, the integrand is divergent as $T \rightarrow \infty$. This has its genesis in implementing (cf. (134))

$$
\frac{1}{p^{2}-m^{2}}=\int_{0}^{\infty} d T e^{-T\left(p^{2}-m^{2}\right)}
$$

and can be remedied by taking instead

$$
\frac{1}{p^{2}-m^{2}}=-\int_{0}^{\infty} d T e^{T\left(p^{2}-m^{2}\right)}
$$

which is equivalent to replacing $T \rightarrow-T$ everywhere. Then, one is then necessarily working in the $p^{2} \leq 0$ regime, in order to make the above integral convergent. So now, one has

$$
-\int_{0}^{\infty} d T \frac{e^{-m^{2} T}}{(4 \pi T)^{2}} e^{\int_{0}^{1} d t\left((1 / 4 T)\left(x-x^{\prime}\right)^{2}+J(t) \cdot\left(x+\left(x-x^{\prime}\right) t\right)\right)+T \int_{0}^{1} d t d t^{\prime}\left(J^{\mu}(t) \eta_{\mu \nu} \Delta\left(t, t^{\prime}\right) J^{\nu}\left(t^{\prime}\right)\right)}
$$

As a result, it is implicit in our assumptions that $\left(x-x^{\prime}\right)^{2} \leq$ 0 ; that is, the spacetime interval can only be spacelike or null, which is compatible with $p^{2} \leq 0$. Thus one is dealing with the offshell propagator, where $[29,46]$

$$
\Delta\left(t, t^{\prime}\right)=t t^{\prime}+\frac{1}{2}\left|t-t^{\prime}\right|-\frac{1}{2}\left(t+t^{\prime}\right)
$$

The $n$-photon amplitude then becomes

$$
\begin{aligned}
& \mathscr{A}\left(x^{\prime}, x ; \varepsilon_{1}, k_{1}, \ldots, \varepsilon_{n}, k_{n}\right)=-\int_{0}^{\infty} d T \frac{e^{-m^{2} T}}{(4 \pi T)^{2}} \exp \left[\int_{0}^{1} d t \frac{1}{4 T}\left(x-x^{\prime}\right)^{2}\right] \times \prod_{i=1}^{n} \int d t_{i} \exp \left[\int_{0}^{1} d t\right. \\
& \cdot \sum_{i=1}^{n}\left(i k_{i \mu}+\varepsilon_{i \mu} \partial_{t_{i}}\right) \delta\left(t-t_{i}\right)\left(x^{\mu}+\left(x-x^{\prime}\right)^{\mu} t\right) \\
& \left.\cdot \int_{0}^{1} d t d t^{\prime} T\left(\sum_{i=1}^{n}\left(i k_{i \mu}+\varepsilon_{i \mu} \partial_{t_{i}}\right) \delta\left(t-t_{i}\right) \eta^{\mu \nu} \Delta\left(t, t^{\prime}\right) \sum_{j=1}^{n}\left(i k_{j v}+\varepsilon_{j v} \partial_{t_{j}}\right) \delta\left(t^{\prime}-t_{j}\right)\right)\right] \\
& =-\int_{0}^{\infty} d T \frac{e^{-m^{2} T+(1 / 4 T)\left(x-x^{\prime}\right)^{2}}}{(4 \pi T)^{2}} \\
& \cdot \prod_{i=1}^{n} \int d t_{i}\left[\left.\exp \left[\sum_{i=1}^{n}\left(i k_{i} \cdot\left(x^{\prime}+\left(x-x^{\prime}\right) t_{i}\right)+\varepsilon_{i} \cdot\left(x-x^{\prime}\right)\right)\right] \exp \left[-T \sum_{i, i^{\prime}=1}^{n}\left(k_{i} \cdot k_{i^{\prime}} \Delta_{i i^{\prime}}-i 2 \varepsilon_{i} \cdot k_{i^{\prime}} \cdot \Delta_{i i^{\prime}}-\varepsilon_{i} \cdot \varepsilon_{i^{\prime}} \cdot \Delta_{i i^{\prime}}\right)\right]\right|_{\text {m.l. }}\right] \text {, }
\end{aligned}
$$

where $\Delta_{i i^{\prime}}=\Delta\left(t_{i}, t_{i^{\prime}}\right)$ and where the dot on the LHS implies derivative with respect to the first argument and that on the right implies with respect to the second one

$$
\begin{gathered}
\cdot \Delta\left(t, t^{\prime}\right)=t^{\prime}-\theta\left(t^{\prime}-t\right), \\
\Delta^{\bullet}\left(t, t^{\prime}\right)=1-\delta\left(t-t^{\prime}\right) .
\end{gathered}
$$

Now, as argued in [46], upon taking Fourier transforms

$$
\begin{aligned}
& \mathscr{A}\left(p^{\prime}, p ; \varepsilon_{1}, k_{1}, \ldots, \varepsilon_{n}, k_{n}\right)=\int d^{D} x \\
& \quad \cdot \int d^{D} x^{\prime} e^{i\left(p \cdot x+p^{\prime} \cdot x^{\prime}\right)} \mathscr{A}\left(x^{\prime}, x ; \varepsilon_{1}, k_{1}, \ldots, \varepsilon_{n}, k_{n}\right)
\end{aligned}
$$

$$
\begin{aligned}
& =\int d^{D} x_{+} \int d^{D} x_{-} e^{i\left(p+p^{\prime}\right) \cdot x_{+}+(i / 2)\left(p-p^{\prime}\right) \cdot x_{-}} \mathscr{A}\left(x_{+}\right. \\
& \left.-\frac{x_{-}}{2}, x_{+}+\frac{x_{-}}{2} ; \varepsilon_{1}, k_{1}, \ldots, \varepsilon_{n}, k_{n}\right),
\end{aligned}
$$

with $x_{-}:=x-x^{\prime}$ and $x_{+}:=\left(x+x^{\prime}\right) / 2$, the integral over the "center of mass" $x_{+}$yields the energy-momentum conservation delta function $(2 \pi)^{D} \delta^{(D)}\left(p+p^{\prime}+\sum k_{i}\right)$, whereas the integral over the "distance" $x_{-}$is Gaussian. Hence, after some simple manipulations, the amplitude reduces to

$$
\begin{aligned}
& \mathscr{A}\left(p^{\prime}, p ; \varepsilon_{1}, k_{1}, \ldots, \varepsilon_{n}, k_{n}\right)=-(i g)^{2}(2 \pi)^{D} \delta^{(D)}\left(p+p^{\prime}+\sum k_{i}\right) \int_{0}^{\infty} d T e^{T\left(p^{2}-m^{2}\right)} \\
& \quad \cdot \prod_{i=1}^{2} \int_{0}^{1} d t_{i} \times\left.\exp \left\{-T(2 p) \cdot \sum_{i=1}^{2}\left(-k_{i} t_{i}+i \varepsilon_{i}\right)-T \sum_{i, j=1}^{n}\left(k_{i} \cdot k_{j}\left(\frac{\left|t_{i}-t_{j}\right|}{2}-\frac{\left(t_{i}+t_{j}\right)}{2}\right)-i \varepsilon_{i} \cdot k_{j}\left(\operatorname{sign}\left(t_{i}-t_{j}\right)-1\right)+\varepsilon_{i} \cdot \varepsilon_{j} \delta\left(t_{i}-t_{j}\right)\right)\right\}\right|_{\mathrm{m} . \mathrm{l} .}
\end{aligned}
$$


since we are working in the spacelike/null region, $p^{2} \leq 0$, and hence $e^{T\left(p^{2}-m^{2}\right)}$ is not divergent as $T \rightarrow \infty$.

Let us now come to the case of the derivation of the vertex in spinor QED. Let us first state what we expect. Since the vertex is just $\gamma^{\mu}$, when a plane-wave photon and two external electron propagators couple to it, we in general expect the momentum space expression

$$
\begin{aligned}
i g(2 \pi)^{D} \delta^{(D)}\left(p+p^{\prime}+k\right) \frac{1}{(\not p-m)} \not \frac{1}{\left(\not p^{\prime}-m\right)} \\
=-i g(2 \pi)^{D} \delta^{(D)}\left(p+p^{\prime}+k\right) \\
\cdot \frac{(\not p+m) \notin(\not p+\not k-m)}{\left(p^{2}-m^{2}\right)\left((p+k)^{2}-m^{2}\right)}
\end{aligned}
$$

$$
\begin{aligned}
& \int_{x_{\mu}(0)=x_{\mu}}^{x_{\mu}(1)=x_{\mu}^{\prime}} \mathscr{D} x \int_{\psi_{i}(0)=\psi_{1 i}}^{\bar{\psi}_{i}(1)=\bar{\psi}_{2 i}} \mathscr{D} \psi \mathscr{D} \bar{\psi} \\
& \quad \cdot \int \frac{\mathscr{D} \beta \mathscr{D e}}{\operatorname{Vol}(\mathrm{Sym})} \exp \left[\left.\int_{0}^{1} d t\left(-\frac{1}{2 e}\left(\dot{x}_{\mu}-\beta \psi_{\mu}\right)^{2}-i \beta m^{\prime}+\frac{1}{2} e m^{2}-\bar{\psi}_{j} \dot{\psi}_{j}-i g \dot{x}^{\mu} A_{\mu}+\frac{i}{2} e g \psi^{\mu} F_{\mu \nu} \psi^{\nu}+\bar{\psi}_{j} \psi_{j}(1)\right]\right|_{\operatorname{lin}},\right.
\end{aligned}
$$

where, as in the previous section, $m^{\prime}=m / \sqrt{2}$. The overall subscript lin implies that only the linear order term in the expansion of the exponent of the interaction term $-i g \dot{x}^{\mu} A_{\mu}+$ $(i / 2) e g \psi^{\mu} F_{\mu \nu} \psi^{\nu}$ is to be retained, as the dressing is only by a single photon. It has all the right ingredients for reproducing the above expression, as we will see, and it is essentially (137), adapted to the case of the Abelian background. The new vertex operators are (upon fixing $e$ to $-2 T$ )

$$
\begin{aligned}
& \left(i g \dot{x}^{\mu} A_{\mu}+\frac{i}{2} e g \psi^{\mu} F_{\mu \nu} \psi^{\nu}\right) \\
& \quad=i g[\varepsilon \cdot \dot{x}(t)-2 i T \varepsilon \cdot \psi(t) k \cdot \psi(t)] e^{i k \cdot x(t)}
\end{aligned}
$$

The relevant amplitude involving just one photon is (upon fixing $\beta$ to $\theta$ and integrating over it)

$$
-\int_{0}^{\infty} d T e^{-m^{2} T} \int_{0}^{1} d t_{1} d t_{2}\left\langle\left[-\frac{1}{2 T} \psi\left(t_{1}\right) \cdot \dot{x}\left(t_{1}\right)\right.\right.
$$

$$
\begin{aligned}
= & i g\left(-p \cdot \varepsilon(2 \not p+k)+\not\left(p^{2}+p \cdot k-m^{2}\right)\right. \\
& \left.+i \gamma^{\sigma} \gamma^{5} \epsilon_{\sigma \mu \nu \rho} p^{\mu} \varepsilon^{v} k^{\rho}+2 m \varepsilon \cdot p-i m \sigma^{\mu \nu} \varepsilon_{\mu} k_{\nu}\right) \\
& \times \int_{0}^{1} d t \frac{1}{\left(p^{2}-m^{2}+2 p \cdot k t\right)^{2}},
\end{aligned}
$$

where in the last line we have used Feynman reparametrization to represent the denominator, with $k^{2}=0$, assuming that the photon is on-shell. Also we have used $\varepsilon \cdot k=0$ in the numerator, implying that we are working in the Lorentz gauge and suppressed the momentum conserving delta function. As we can see, this expression is formidable, and we claim that the following is its worldline path-integral representation in the coherent state basis:

$$
\begin{aligned}
& \left.-i m^{\prime}\right]\left[i g\left[\varepsilon \cdot \dot{x}\left(t_{2}\right)-2 i T \varepsilon \cdot \psi\left(t_{2}\right) k \cdot \psi\left(t_{2}\right)\right]\right. \\
& \left.\left.\cdot e^{i k \cdot x\left(t_{2}\right)}\right]\right\rangle .
\end{aligned}
$$

Let us first work out the $x$ parts.

This would be given by

$$
\begin{aligned}
& -(\text { ig }) \int_{0}^{\infty} d T e^{-m^{2} T} \int d \theta \\
& .\left.\int_{0}^{1} d t_{2}\left\langle e^{\int d t\left(J(t) \cdot x(t)-(\theta / 2 T) \int d t_{1} \psi\left(t_{1}\right) \cdot \dot{x}\left(t_{1}\right)\right)}\right\rangle\right|_{\operatorname{lin} .}
\end{aligned}
$$

with

$$
J_{\mu}(t)=\left(i k_{\mu}+\varepsilon_{\mu} \partial_{t_{2}}\right) \delta\left(t-t_{2}\right)
$$

The general amplitude involving multiple photons and the susy charge operator $\psi\left(t_{1}\right) \cdot \dot{x}\left(t_{1}\right)$, upon Fourier transforming to momentum space, is

$$
\begin{aligned}
& \mathscr{A}\left(p^{\prime}, p ; \varepsilon_{1}, k_{1}, \ldots, \varepsilon_{n}, k_{n}\right)=-(i g)^{2}(2 \pi)^{D} \delta^{(D)}\left(p+p^{\prime}+\sum k_{i}\right) \int_{0}^{\infty} d T e^{T\left(p^{2}-m^{2}\right)} \\
& \quad \cdot \prod_{i=1}^{n} \int_{0}^{1} d t_{i} \times\left.\exp \left\{-T(2 p) \cdot \sum_{i=1}^{n}\left(-k_{i} t_{i}+i \varepsilon_{i}\right)-T \sum_{i, j=1}^{n}\left(k_{i} \cdot k_{j}\left(\frac{\left|t_{i}-t_{j}\right|}{2}-\frac{\left(t_{i}+t_{j}\right)}{2}\right)-i \varepsilon_{i} \cdot k_{j}\left(\operatorname{sign}\left(t_{i}-t_{j}\right)-1\right)+\varepsilon_{i} \cdot \varepsilon_{j} \delta\left(t_{i}-t_{j}\right)\right)\right\}\right|_{\mathrm{m} .1} \\
& \quad \times\left\langle\left[i \int_{0}^{1} d t_{1} \psi\left(t_{1}\right) \cdot p+i \sum_{i} \int_{0}^{t_{i}} d t_{1} \psi_{\mu}\left(t_{1}\right) k_{i}^{\mu}+\sum_{i} \psi_{\mu}\left(t_{i}\right) \varepsilon_{i}^{\mu}\right] \ldots\right\rangle
\end{aligned}
$$


where we have done the $\theta$ integral and where the ellipses denote other $\psi$ insertions that might be present. Here we have simply adapted the analysis above for scalars to include the effects of the additional source in (159). The expectation value in the last line is with respect to the $\psi$ path-integral. So at this point we need to evaluate the $\psi$ correlators. As is evident from (158), we would need 1,2, and 3 point $\psi$ correlators. The point made is that we now need the correlators for the Majorana fermions $\psi_{\mu}$, which we have to derive from the Dirac form of the action, as it is the only form amenable for the coherent state formalism. The relevant path-integral is given by

$$
\int_{\psi_{i}(0)=\psi_{1 i}}^{\bar{\psi}_{i}(1)=\bar{\psi}_{2 i}} \mathscr{D} \psi \mathscr{D} \bar{\psi} e^{\int_{0}^{1} d t\left(-\bar{\psi}_{i} \dot{\psi}_{i}+\bar{\chi}_{i} \psi_{i}+\chi_{i} \bar{\psi}_{i}\right)+\bar{\psi}_{i} \psi_{i}(1)} .
$$

Again, $\psi$ 's can be split into background and quantum parts as follows:

$$
\begin{aligned}
& \bar{\psi}_{i}=\bar{\psi}_{2 i}+\bar{\psi}_{q i}, \\
& \psi_{i}=\psi_{1 i}+\psi_{q i}
\end{aligned}
$$

and the resultant path-integral looks like

$$
e^{\bar{\psi}_{2 i} \psi_{1 i}} \int_{\psi_{q i}(0)=0}^{\bar{\psi}_{q i}(1)=0} \mathscr{D} \psi \mathscr{D} \bar{\psi} e^{\int_{0}^{1} d t\left(-\bar{\psi}_{q i} \dot{\psi}_{q i}+\bar{\chi}_{i}\left(\psi_{1 i}+\psi_{q i}\right)+\left(\bar{\psi}_{2 i}+\bar{\psi}_{q i}\right) \chi_{i}\right)}
$$

where the prefactor is the usual normalization factor. Upon integration, the above becomes (cf. Appendix A (see (A.18)))

$$
=e^{\bar{\psi}_{2 i} \psi_{1 i}} e^{-\left[\int_{0}^{1} d t d t^{\prime}\left(\bar{\chi}_{i}(t) \theta\left(t-t^{\prime}\right) \chi_{i}\left(t^{\prime}\right)\right)-\int_{0}^{1} d t\left(\bar{\chi}_{i}(t) \psi_{1 i}+\bar{\psi}_{2 i} \chi_{i}(t)\right)\right]}
$$

and this can be written in the Majorana basis as

$$
e^{\bar{\psi}_{2 i} \psi_{1 i}} \exp \left[\int_{0}^{1} d t\left(\chi(t) \cdot \psi_{b}\right)-\frac{1}{4} \int_{0}^{1} d t d t^{\prime}\left(\chi_{\mu}(t)\left[\eta^{\mu \nu}\left[\theta\left(t^{\prime}-t\right)-\theta\left(t-t^{\prime}\right)\right]+K^{\mu \nu}\right] \chi_{\nu}\left(t^{\prime}\right)\right)\right] .
$$

Here $\chi_{\mu}$ are precisely the sources for the Majorana fermions $\psi_{\mu}$, where $\chi^{\mu}$ is related to $\bar{\chi}_{i}$ and $\chi_{i}$ as

$$
\begin{aligned}
& \chi^{0}=\frac{1}{\sqrt{2}}\left(\bar{\chi}_{1}-\chi_{1}\right), \\
& \chi^{3}=\frac{1}{\sqrt{2}}\left(\bar{\chi}_{1}+\chi_{1}\right), \\
& \chi^{2}=\frac{i}{\sqrt{2}}\left(\bar{\chi}_{2}-\chi_{2}\right), \\
& \chi^{1}=\frac{1}{\sqrt{2}}\left(\bar{\chi}_{2}+\chi_{2}\right)
\end{aligned}
$$

and $\psi_{b}^{\mu}$ is given by

$$
\begin{aligned}
& \psi_{b}^{0}=\frac{1}{\sqrt{2}}\left(\bar{\psi}_{21}+\psi_{11}\right), \\
& \psi_{b}^{3}=\frac{1}{\sqrt{2}}\left(\bar{\psi}_{21}-\psi_{11}\right), \\
& \psi_{b}^{2}=\frac{i}{\sqrt{2}}\left(\psi_{12}+\bar{\psi}_{22}\right), \\
& \psi_{b}^{1}=\frac{1}{\sqrt{2}}\left(\bar{\psi}_{22}-\psi_{12}\right) .
\end{aligned}
$$

Also,

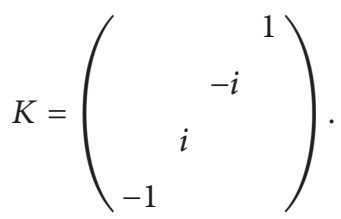

Let us first tackle 3-point correlator. It is given by

$$
\begin{gathered}
\left\langle\psi^{\alpha}\left(t_{1}\right) \psi^{\beta}\left(t_{2}\right) \psi^{\gamma}\left(t_{2}\right)\right\rangle=\frac{\partial}{\partial \chi_{\alpha}\left(t_{1}\right)} \frac{\partial}{\partial \chi_{\beta}\left(t_{2}\right)} \frac{\partial}{\partial \chi_{\gamma}\left(t_{2}\right)} \\
e^{\bar{\psi}_{2 i} \psi_{1 i}} \exp \left[\int_{0}^{1} d t\left(\chi(t) \cdot \psi_{b}\right)+\frac{1}{4}\right. \\
\left.\cdot \int_{0}^{1} d t d t^{\prime}\left(\chi_{\mu}(t)\left[\eta^{\mu \nu}\left[\operatorname{sign}\left(t-t^{\prime}\right)\right]-K^{\mu \nu}\right] \chi_{\nu}\left(t^{\prime}\right)\right)\right]\left.\right|_{\chi=0} \\
=e^{\bar{\psi}_{2 i} \psi_{1 i}}\left[\frac{1}{2} K^{\beta \gamma} \psi_{b}^{\alpha}+\frac{1}{2}\left(\eta^{\alpha \gamma}\left[\operatorname{sign}\left(t_{1}-t_{2}\right)\right]-K^{\alpha \gamma}\right) \psi_{b}^{\beta}\right. \\
\left.-\frac{1}{2} \psi_{b}^{\gamma}\left(\eta^{\alpha \beta}\left[\operatorname{sign}\left(t_{1}-t_{2}\right)\right]-K^{\alpha \beta}\right)-\psi_{b}^{\gamma} \psi_{b}^{\beta} \psi_{b}^{\alpha}\right] .
\end{gathered}
$$

It can be easily checked that, upon transforming to the Dirac basis, it assumes the form

$$
\begin{aligned}
& \frac{1}{2 \sqrt{2}}\left[\left(\eta^{\alpha \gamma}\left[\operatorname{sign}\left(t_{1}-t_{2}\right)\right]\right) \gamma^{\beta}\right. \\
& \left.\quad-\gamma^{\gamma}\left(\eta^{\alpha \beta}\left[\operatorname{sign}\left(t_{1}-t_{2}\right)\right]\right)-\gamma^{\gamma} \gamma^{\beta} \gamma^{\alpha}\right]
\end{aligned}
$$

with $\gamma, \beta, \alpha$ all different in the last term, in which case it can be written as

$$
\begin{aligned}
& \frac{1}{2 \sqrt{2}}\left[\left(\eta^{\alpha \gamma}\left[\operatorname{sign}\left(t_{1}-t_{2}\right)\right]\right) \gamma^{\beta}\right. \\
& \left.\quad-\gamma^{\gamma}\left(\eta^{\alpha \beta}\left[\operatorname{sign}\left(t_{1}-t_{2}\right)\right]\right)+i \epsilon^{\sigma \gamma \beta \alpha} \gamma_{\sigma} \gamma^{5}\right] .
\end{aligned}
$$


In order to derive this, one has to use (168), (11) and (14), and the following:

$$
\begin{aligned}
& \widehat{\psi}^{0}=\frac{1}{\sqrt{2}}\left(\widehat{\bar{\psi}}_{1}+\widehat{\psi}_{1}\right), \\
& \widehat{\psi}^{3}=\frac{1}{\sqrt{2}}\left(\widehat{\bar{\psi}}_{1}-\widehat{\psi}_{1}\right), \\
& \widehat{\psi}^{2}=\frac{i}{\sqrt{2}}\left(\widehat{\psi}_{2}+\widehat{\bar{\psi}}_{2}\right), \\
& \widehat{\psi}^{1}=\frac{1}{\sqrt{2}}\left(\widehat{\bar{\psi}}_{2}-\widehat{\psi}_{2}\right) .
\end{aligned}
$$

We also have to use the facts that, for instance,

$$
\begin{aligned}
\left\langle\bar{\psi}_{2}\left|\widehat{\psi}_{i} \hat{\bar{\psi}}_{j}\right| \psi_{1}\right\rangle & =\left\langle\bar{\psi}_{2}\left|\left(\delta_{i j}-\widehat{\bar{\psi}}_{j} \widehat{\psi}_{i}\right)\right| \psi_{1}\right\rangle \\
& =e^{\bar{\psi}_{2 i} \psi_{1 i}}\left(\delta_{i j}-\bar{\psi}_{2 j} \psi_{1 i}\right) \\
& =e^{\bar{\psi}_{2 i} \psi_{1 i}}\left(\delta_{i j}+\psi_{1 i} \bar{\psi}_{2 j}\right)
\end{aligned}
$$

and hence when three different $\widehat{\psi}^{\mu}$ 's are involved,

$$
\begin{aligned}
& \left\langle\bar{\psi}_{2}\left|\widehat{\psi}^{\gamma} \widehat{\psi}^{\beta} \widehat{\psi}^{\alpha}\right| \psi_{1}\right\rangle=e^{\bar{\psi}_{2 i} \psi_{1 i}}\left(-\frac{1}{2} K^{\beta \gamma} \psi_{b}^{\alpha}+\frac{1}{2} K^{\alpha \gamma} \psi_{b}^{\beta}\right. \\
& \left.-\frac{1}{2} \psi_{b}^{\gamma} K^{\alpha \beta}+\psi_{b}^{\gamma} \psi_{b}^{\beta} \psi_{b}^{\alpha}\right)
\end{aligned}
$$

with the expression vanishing if any of $\widehat{\psi}$ 's are the same, thus automatically antisymmetrizing the expressions, leading to $(1 / 2 \sqrt{2}) \gamma^{\gamma} \gamma^{\beta} \gamma^{\alpha}$ in the Dirac basis, since $\widehat{\psi}^{\mu}$ in the Dirac basis becomes $(1 / \sqrt{2}) \gamma^{\mu}$. Next, let us come to the 2-point correlator. It is given by

$$
\begin{aligned}
& \left\langle\psi^{\beta}\left(t_{2}\right) \psi^{\gamma}\left(t_{2}\right)\right\rangle \\
& =\left.\frac{\partial}{\partial \chi_{\beta}\left(t_{2}\right)} \frac{\partial}{\partial \chi_{\gamma}\left(t_{2}\right)} e^{\bar{\psi}_{2 i} \psi_{1 i}} \exp \left[\int_{0}^{1} d t\left(\chi(t) \cdot \psi_{b}\right)+\frac{1}{4} \int_{0}^{1} d t d t^{\prime}\left(\chi_{\mu}(t)\left[\eta^{\mu \nu}\left[\operatorname{sign}\left(t-t^{\prime}\right)\right]-K^{\mu \nu}\right] \chi_{\nu}\left(t^{\prime}\right)\right)\right]\right|_{\chi=0} \\
& =e^{\bar{\psi}_{2 i} \psi_{1 i}}\left[\frac{1}{2} K^{\beta \gamma}-\psi_{b}^{\gamma} \psi_{b}^{\beta}\right] \simeq \frac{i}{2} \sigma^{\gamma \beta}
\end{aligned}
$$

and the one-point function is simply

$$
\left\langle\psi^{\mu}(t)\right\rangle \simeq \frac{1}{\sqrt{2}} \gamma^{\mu}
$$

So we are now ready to tackle (161) for one photon, which corresponds to the following part of (158):

$$
\begin{gathered}
\int_{0}^{\infty} d T \int_{0}^{1} d t_{1} d t_{2}\left\langle\left[\frac{1}{2 T} \psi\left(t_{1}\right) \cdot \dot{x}\left(t_{1}\right)\right]\right. \\
\left.\cdot\left[i g \varepsilon \cdot \dot{x}\left(t_{2}\right) e^{i k \cdot x\left(t_{2}\right)}\right]\right\rangle
\end{gathered}
$$

and it would be

$$
\begin{aligned}
& -(i g)(2 \pi)^{D} \delta^{(D)}\left(p+p^{\prime}+k\right) \int_{0}^{\infty} d T e^{T\left(p^{2}-m^{2}\right)} \\
& \quad \cdot \int_{0}^{1} d t_{2} \times\left.\exp \left\{\left(2 T p \cdot k t_{2}-i 2 T p \cdot \varepsilon\right)\right\}\left\langle\left[i \int_{0}^{1} d t_{1} \psi\left(t_{1}\right) \cdot p+i \int_{0}^{t_{2}} d t_{1} \psi_{\mu}\left(t_{1}\right) k^{\mu}+\psi_{\mu}\left(t_{2}\right) \varepsilon^{\mu}\right]\right\rangle\right|_{\text {lin. }},
\end{aligned}
$$

where lin means linear in $\varepsilon^{\mu}$. Using the one-point function above, (178), we would get (upon extracting the part linear in $\varepsilon)$

$$
\begin{aligned}
& \mathscr{A}\left(p^{\prime}, p ; \varepsilon, k\right)=-\frac{1}{\sqrt{2}}(i g)(2 \pi)^{D} \delta^{(D)}\left(p+p^{\prime}+k\right) \\
& \cdot \int_{0}^{\infty} d T e^{T\left(p^{2}-m^{2}\right)} \\
& \quad \cdot \int_{0}^{1} d t_{2} \times \exp \left(2 T p \cdot k t_{2}\right)\left[2 T p \cdot \varepsilon\left(\not p+k t_{2}\right)+\not\right],
\end{aligned}
$$

$$
\begin{aligned}
= & i g \sqrt{2} \int_{0}^{1} d t_{2} \frac{1}{\left(p^{2}-m^{2}+2 p \cdot k t_{2}\right)^{2}} \\
& \cdot\left[-p \cdot \varepsilon\left(\not p+k t_{2}\right)+\frac{1}{2} \not\left(p^{2}-m^{2}+2 p \cdot k t_{2}\right)\right],
\end{aligned}
$$

where we have suppressed the delta function. Let us then come to the first mass term in (158), namely,

$$
-g m^{\prime} \int_{0}^{\infty} d T \int_{0}^{1} d t_{1} d t_{2}\left\langle\varepsilon \cdot \dot{x}\left(t_{2}\right) e^{i k \cdot x\left(t_{2}\right)}\right\rangle
$$


Using the master formula (161), we have

$$
\begin{aligned}
& -\left(g m^{\prime}\right) \int_{0}^{\infty} d T e^{T\left(p^{2}-m^{2}\right)} \\
& \left.\cdot \int_{0}^{1} d t_{2} \exp \left\{\left(2 T p \cdot k t_{2}+i 2 T p \cdot \varepsilon\right)\right\}\right|_{\text {lin. }} e^{\bar{\psi}_{2 i} \psi_{1 i}} \\
& =i g m \sqrt{2} \int_{0}^{1} d t_{2} \frac{1}{\left(p^{2}-m^{2}+2 p \cdot k t_{2}\right)^{2}} p \cdot \varepsilon e^{\bar{\psi}_{2 i} \psi_{1 i}},
\end{aligned}
$$

where $e^{\bar{\psi}_{2 i} \psi_{1 i}}$ is the ubiquitous part coming from the fermion path-integral, which means that when one transforms to the Dirac basis, it would yield identity for this term. Next let us consider the following term in (158):

$$
\begin{gathered}
-i g \int_{0}^{\infty} d T \int_{0}^{1} d t_{2} d t_{1}\left\langle\left[\frac{1}{2 T} \psi\left(t_{1}\right) \cdot \dot{x}\left(t_{1}\right)\right]\right. \\
\left.\cdot\left[2 i T \varepsilon \cdot \psi\left(t_{2}\right) k \cdot \psi\left(t_{2}\right) e^{i k \cdot x\left(t_{2}\right)}\right]\right\rangle .
\end{gathered}
$$

Again, using the master formula (161) for the $x$-part, we get

$$
-2 g \int_{0}^{\infty} d T e^{T\left(p^{2}-m^{2}\right)} T \int_{0}^{1} d t_{2} \exp \left(2 T p \cdot k t_{2}\right)
$$

$$
\begin{aligned}
& \cdot\left\langle\left[i \int d t_{1} \psi\left(t_{1}\right) \cdot p+i \int_{0}^{t_{2}} d t_{1} \psi_{\mu}\left(t_{1}\right) k^{\mu}\right]\right. \\
& \left.\cdot\left[\varepsilon \cdot \psi\left(t_{2}\right) k \cdot \psi\left(t_{2}\right)\right]\right\rangle .
\end{aligned}
$$

Now, upon using (172) and

$$
\begin{gathered}
\int_{0}^{1} d t_{1} d t_{2} \operatorname{sign}\left(t_{1}-t_{2}\right) f\left(t_{2}\right) \\
=\int_{0}^{1} d t_{2}\left(2 t_{2}-1\right) f\left(t_{2}\right)
\end{gathered}
$$

we get

$$
\begin{aligned}
- & 2 i g \int_{0}^{1} d t_{2} \frac{1}{\left(p^{2}-m^{2}+2 p \cdot k t_{2}\right)^{2}} \\
& \cdot \frac{1}{2 \sqrt{2}}\left[\notin\left(p \cdot k\left(2 t_{2}-1\right)\right)-p \cdot \varepsilon\left(k\left(2 t_{2}-1\right)\right)\right. \\
& \left.+i \epsilon^{\sigma \gamma \beta \alpha} \gamma_{\sigma} \gamma^{5} p_{\alpha} \varepsilon_{\beta} k_{\gamma}\right],
\end{aligned}
$$

where we have used $k \cdot \varepsilon=k^{2}=\epsilon^{\sigma \gamma \beta \alpha} \gamma_{\sigma} \gamma^{5} k_{\alpha} \varepsilon_{\beta} k_{\gamma}=0$. This when combined with (182) yields

$$
i g \frac{1}{\sqrt{2}} \int_{0}^{1} d t_{2} \frac{1}{\left(p^{2}-m^{2}+2 p \cdot k t_{2}\right)^{2}}\left[-p \cdot \varepsilon(2 \not p+k)+\not\left(p^{2}-m^{2}+p \cdot k\right)-i \epsilon^{\sigma \gamma \beta \alpha} \gamma_{\sigma} \gamma^{5} p_{\alpha} \varepsilon_{\beta} k_{\gamma}\right] .
$$

Now we come to the remaining term in (158):

$$
\begin{aligned}
& g m^{\prime} \int_{0}^{\infty} d T \\
& \quad \cdot \int_{0}^{1} d t_{2} d t_{1}\left\langle\left[2 i T \varepsilon \cdot \psi\left(t_{2}\right) k \cdot \psi\left(t_{2}\right)\right] e^{i k \cdot x\left(t_{2}\right)}\right\rangle
\end{aligned}
$$

which upon using the master formula for the $x$-part and using (177) yields

$$
\begin{aligned}
& -\left(g m^{\prime}\right) \int_{0}^{\infty} d T e^{T\left(p^{2}-m^{2}\right)} T \int_{0}^{1} d t_{2} \exp \left(2 T p \cdot k t_{2}\right) \\
& \cdot \sigma^{\gamma \beta} \varepsilon_{\beta} k_{\gamma} \\
& =i g \sqrt{2} m \int_{0}^{1} d t_{2} \frac{1}{\left(p^{2}-m^{2}+2 p \cdot k t_{2}\right)^{2}} \frac{i}{2} \sigma^{\gamma \beta} \varepsilon_{\beta} k_{\gamma} .
\end{aligned}
$$

Thus collecting terms from (185), (190), and (193), we finally end up with

$$
\begin{aligned}
& i g \frac{1}{\sqrt{2}} \int_{0}^{1} d t_{2} \frac{1}{\left(p^{2}-m^{2}+2 p \cdot k t_{2}\right)^{2}}\left[i m \sigma^{\gamma \beta} \varepsilon_{\beta} k_{\gamma}-p\right. \\
& \cdot \varepsilon(2 \not p+\not k)+\not\left(p^{2}-m^{2}+p \cdot k\right) \\
&\left.\quad-i \epsilon^{\sigma \gamma \beta \alpha} \gamma_{\sigma} \gamma^{5} p_{\alpha} \varepsilon_{\beta} k_{\gamma}+2 m p \cdot \varepsilon\right]
\end{aligned}
$$

which is precisely the same as (155) up to a factor of $1 / \sqrt{2}$. Now that we essentially have (153), we can amputate the external legs and get $\varepsilon^{\mu} \gamma_{\mu}$ from which the vertex can be obtained by taking derivative with respect to $\varepsilon^{\mu}$. We have thus obtained the QED Feynman rule for the vertex starting from the worldline formalism.

The obvious next step would be computing the Compton scattering amplitude, which is a repeat of the above calculation with two photon vertex operators instead of one in (158). But since the single photon case is formidable enough, we are currently investigating methods that can vastly simplify the calculations, allowing us to tackle even more involved cases like the Lamb-shift calculation. One can then also venture into the applications to the non-Abelian case. 


\section{One-Loop Effective Actions}

Let us now consider the analogous representations for oneloop effective actions and anomalies. The usual one-loop effective action for a fermionic field in a gauge field background is given by

$$
\begin{array}{rr}
\frac{1}{2} \ln \operatorname{Det}\left[-(\gamma \cdot D)^{2}\right] & =-\frac{1}{2} \operatorname{Tr} \int_{0}^{\infty} \frac{d T}{T} \exp \left\{-T\left[-D^{2}-\frac{1}{2} g \sigma^{\mu \nu} F_{\mu \nu}^{a} T^{a}\right]\right\} \quad \text { which has the following path-integral representation [5]: } \\
=\frac{1}{2} \ln \operatorname{Det}\left[-D^{2}-\frac{1}{2} g \sigma^{\mu \nu} F_{\mu \nu}^{a} T^{a}\right] & \quad \text { w } \\
- & \frac{1}{2} \int \frac{d T}{T} \int_{P} \mathscr{D} x \int_{\mathrm{AP}} \mathscr{D} \psi \mathscr{D} \bar{\psi} e^{\int_{0}^{1} d t\left(-(1 / 4 T) \dot{x}^{2}-\bar{\psi}_{j} \dot{\psi}_{j}\right)} \operatorname{Tr} P e^{i \int d t\left(g \dot{x}^{\mu} A_{\mu}^{a} T^{a}+T g \psi^{\mu} F_{\mu \nu}^{a} T^{a} \psi^{\nu}\right)},
\end{array}
$$

where $D_{\mu}=\partial_{\mu}+i g A_{\mu}^{a} T^{a}$ and can be expressed as where the trace is over a super-Wilson loop that is the worldline supersymmetric generalization of the normal Wilson loop. $P$ denotes periodic, while AP denotes antiperiodic.
Now it can be shown that the effective action of a Dirac fermion that transforms as an $S U(5)$ tensor of rank $n$ would be given by the following path-integral (see [29-34] for related approaches):

$$
\int_{P} \mathscr{D} x \int_{\mathrm{AP}} \mathscr{D} \psi \mathscr{D} \bar{\psi} \int_{\mathrm{AP}} \mathscr{D} \lambda \mathscr{D} \bar{\lambda} \int \mathscr{D} a \times \int \frac{\mathscr{D} e}{\operatorname{Vol}(\mathrm{Sym})} \mathscr{D} p e^{\int_{0}^{1} d t\left(i p^{\mu} \dot{x}_{\mu}-\bar{\psi}_{j} \dot{\psi}_{j}-\bar{\lambda}_{i} \dot{\lambda}_{i}-(1 / 2) e \pi^{\mu} \pi_{\mu}+(i / 2) e g \psi^{\mu} \mathbf{F}_{\mu \nu}^{i j} \bar{\lambda}_{i} \lambda_{j} \psi^{\nu}-i a \bar{\lambda}_{i} \lambda_{i}+i(n-5 / 2) a\right)}
$$

where $\pi_{\mu}=p_{\mu}-g \mathbf{A}_{\mu}^{i j} \bar{\lambda}_{i} \lambda_{j}$ with $\mathbf{A}_{\mu}^{i j}=\left(A_{\mu}^{a} T^{a}\right)^{i j}$ and $\mathbf{F}_{\mu \nu}^{i j}$ is the full self-interacting field strength with $T^{a}$ belonging to the Standard Model gauge group. $\lambda$ 's are 5 in number, as in the above section, in order to accommodate the full SM gauge group. We have dropped the prefactor of $-1 / 2$, which is understood to be implicit in what follows. $\beta$, the auxiliary field multiplying the susy generator, has been fixed to zero, since it is antiperiodic and an antiperiodic field has no zero modes. Integrating out $p^{\mu}$ at first yields

$$
\int \frac{d T}{T} \int_{P} \mathscr{D} x \int_{\mathrm{AP}} \mathscr{D} \psi \mathscr{D} \bar{\psi} \int_{\mathrm{AP}} \mathscr{D} \lambda \mathscr{D} \bar{\lambda} \int \mathscr{D} a \times\left(e^{\int_{0}^{1} d t\left(-(1 / 4 T) \dot{x}^{2}+i g \dot{x}^{\mu} \mathbf{A}_{\mu}^{i j} \bar{\lambda}_{i} \lambda_{j}+i T g \psi^{\mu} \mathbf{F}_{\mu \nu}^{i j} \bar{\lambda}_{i} \lambda_{j} \psi^{\nu}-\bar{\psi}_{j} \dot{\psi}_{j}-\bar{\lambda}_{i} \dot{\lambda}_{i}-i a \bar{\lambda}_{i} \lambda_{i}+i(n-5 / 2) a\right)}\right)
$$

where we have fixed $e$ to $2 T$ and for a circle the measure for the modular integral is $1 / T$, which then tallies with (197).

Let us first show that, for a fermion transforming in and only in the fundamental representation of the gauge group $(n=1)$, the integral over $\lambda$ and $\bar{\lambda}$ gives rise precisely to the trace of the super-Wilson loop in (197). We will compute the $\lambda$ and $\bar{\lambda}$ integrals for open intervals first and then identify the endpoints with a change in sign. There is a subtlety involved here in that, for instance, the integral over $T$ for a closed loop for the bosonic part involves a measure factor of $1 / T$ that would be missing if one arrived at the closed loop by simply sewing open lines which do not have such a term, and so one must watch out for such factors here as well. However, in the fermionic case at hand the measures involved are trivial for both closed and open loops and there are no problems ${ }^{4}$.

Let us call $-\left(g \dot{x}^{\mu} \mathbf{A}_{\mu}^{i j}+T g \psi^{\mu} \mathbf{F}_{\mu \nu}^{i j} \psi^{\nu}\right), M_{i j}(t)$. Let us again twist $\lambda$ 's as in (49). Then the open ended path-integral becomes

$$
\int_{\lambda_{i}(0)=\lambda_{i 1}}^{\bar{\lambda}_{i}(1)=\bar{\lambda}_{i 2} e^{-i a}} \mathscr{D} \lambda \mathscr{D} \bar{\lambda} e^{-\int d t\left(\bar{\lambda}_{i}(t)\left(d / d t+i M_{i j}(t)\right) \lambda_{j}(t)\right)},
$$

where the understanding is that, at the end, we will identify $\lambda_{i}(0)=-\lambda_{i}(1)$ and integrate over it. Since this is quadratic in $\lambda$, it can be evaluated. We first make a transformation $M_{i j}(t) \rightarrow U_{i k}^{-1}(t) M_{k l}(t) U_{l j}(t)=M_{i j}^{\prime}(t)$ and $\lambda_{j}(t) \rightarrow$ $U_{j m}^{-1}(t) \lambda_{m}(t)=\lambda_{j}^{\prime}(t)$, and $\bar{\lambda}_{i}(t) \rightarrow \bar{\lambda}_{n}(t) U_{n i}(t)=\bar{\lambda}_{i}^{\prime}(t)$. Since $M$ 's are traceless and Hermitian, $U$ 's are to be chosen to be special unitary, to maintain these properties. Then the Lagrangian transforms as

$$
\begin{aligned}
& \bar{\lambda}_{i}(t)\left(\frac{d}{d t}+i M_{i j}(t)\right) \lambda_{j}(t)=\bar{\lambda}_{n}^{\prime}(t) \\
& \cdot\left(\frac{d}{d t}+U_{n i}^{-1}(t) \frac{d}{d t} U_{i m}(t)+i U_{n k}^{-1}(t) M_{k l} U_{l m}(t)\right) \\
& \cdot \lambda_{m}^{\prime}(t) .
\end{aligned}
$$


We choose $U(t)$ such that

$$
\begin{aligned}
& U_{n i}^{-1}(t) \frac{d}{d t} U_{i m}(t)+i U_{n k}^{-1}(t) M_{k l} U_{l m}(t) \\
& \quad=i M_{n}^{\prime}(t) \delta_{n m}
\end{aligned}
$$

where $\sum_{n} M_{n}^{\prime}=0$, owing to the tracelessness property of $M$ and the specialness of $U$ 's. Thus we have

$$
\frac{d}{d t} U U^{-1}+i M=i U M^{\prime} U^{-1}
$$

or

$$
i M=U \frac{d}{d t} U^{-1}+i U M^{\prime} U^{-1}
$$

Then we have

$$
\begin{gathered}
\int_{\lambda_{i}^{\prime}(0)=\lambda_{i 1}^{\prime}}^{\bar{\lambda}_{i}^{\prime}(1)=\bar{\lambda}_{i 2}^{\prime} e^{-i a}} \mathscr{D} \lambda^{\prime} \mathscr{D} \overline{\lambda^{\prime}} \operatorname{Det}^{-1}\left[U(t) U^{-1}(t)\right] \\
\cdot e^{-\int d t\left(\bar{\lambda}_{i}^{\prime}(t)\left(d / d t+i M_{i}^{\prime}(t)\right) \lambda_{i}^{\prime}(t)\right)} .
\end{gathered}
$$

The Jacobian determinant is 1 . The path-integral becomes (Appendix A) (cf. (A.26))

$$
T\left(e^{(i / 2) \int d t \sum_{i} M_{i}^{\prime}(t)}\right) \prod_{i} e^{\bar{\lambda}_{i 2}^{\prime} e^{-i a} T\left(e^{-i \int d t M_{i}^{\prime}(t)}\right) \lambda_{i 1}^{\prime}-\overline{\lambda_{i 2}^{\prime}} \lambda_{i 2}^{\prime}},
$$

where $T$ implies time-ordering. Now this is equivalent to (Appendix C)

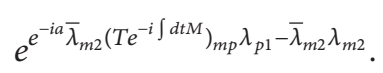

Finally, let us identify the endpoint $\lambda$ 's after incorporating a minus sign, so that the above expression gets converted to, upon reinstating the integrals over the boundary values,

$$
\int d \bar{\lambda} d \lambda \frac{d a}{(2 \pi)} e^{i a} e^{-\left(e^{-i a} \bar{\lambda}_{m}\left(T e^{-i \int d t M}\right)_{m p} \lambda_{p}+\bar{\lambda}_{m} \lambda_{m}\right)},
$$

where one has fixed $a$ to a constant modulus that one continues to call $a$ and used $n=1$. Then doing the $a$ integral begets (since only the $e^{-i a} \bar{\lambda}_{m}\left(T e^{-i \int d t M}\right)_{m p} \lambda_{p}$ term would survive the integration with all higher and lower powers dropping out)

$$
-\int d \bar{\lambda} d \lambda \bar{\lambda}_{m}\left(T e^{-i \int d t M}\right)_{m p} \lambda_{p} e^{-\bar{\lambda}_{m} \lambda_{m}}
$$

which is nothing but

$$
\operatorname{Tr}\left(P e^{-i \int d t M}\right)
$$

where time-ordering has been replaced by path-ordering. Hence, we have proved that, for fundamental fermions, the integral over $\lambda$ 's gives rise to the super-Wilson loop:

$$
\operatorname{Tr}\left(P e^{i \int d t\left(g \dot{x}^{\mu} \mathbf{A}_{\mu}^{i j}+\operatorname{Tg} \psi^{\mu} \mathbf{F}_{\mu \nu}^{i j} \psi^{\nu}\right)}\right)
$$

thus establishing the connection between (198) and (197) for $n=1$ that we set out to prove, and this has been considered as a starting point for introducing the $\lambda$ fermions in some places.

However, as is evident, this will no longer work for fermions of higher rank representations requiring modifications such as those reported in [31]. But there is an economical way of considering the one-loop effective actions for SM fermions. And that is to treat them holistically, namely, considering the loop-running of all the fermions together. The way to consider this is by imposing the condition

$$
(-1)^{\bar{\lambda}_{i} \lambda_{i}}=1
$$

on the spectrum of states. Notice that this condition automatically selects out the representations with even $N_{\lambda}$, namely, the $\mathbf{1 6}$ in Section 6. Thus imposing this condition essentially allows all the fermions to run in the loops, except that the right-handed antineutrino just goes along for a free ride as it is a singlet of the SM and hence does not couple to the gauge bosons. Now, inserting $(1 / 2)\left(1+(-1)^{\bar{\lambda}_{i} \lambda_{i}}\right)$ in a trace over the full state spectrum projects out the states satisfying $(-1)^{\bar{\lambda}_{i} \lambda_{i}}=$ -1 , leaving the states satisfying the above condition only. In the path-integral representation of the trace, this insertion is equivalent to the taking of a sum of the unrestricted effective actions with antiperiodic and periodic boundary conditions for $\lambda$ 's [8]. The expression to be considered is thus

$$
\begin{aligned}
& \frac{1}{4} \int \frac{d T}{T} \int_{P} \mathscr{D} x\left(\int_{\mathrm{AP}}-\int_{P}\right) \mathscr{D} \psi \mathscr{D} \bar{\psi} \times\left(\int_{\mathrm{AP}}+\int_{P}\right) \\
& \cdot \mathscr{D} \lambda \mathscr{D} \bar{\lambda}\left(e^{\int_{0}^{1} d t\left(-(1 / 4 T) \dot{x}^{2}+i g \dot{x}^{\mu} \mathrm{A}_{\mu}^{i j} \bar{\lambda}_{i} \lambda_{j}+i T g \psi^{\mu} \mathrm{F}_{\mu \nu}^{i j} \bar{\lambda}_{i} \lambda_{j} \psi^{\nu}-\bar{\psi}_{j} \dot{\psi}_{j}-\bar{\lambda}_{i} \dot{\lambda}_{i}\right.}\right),
\end{aligned}
$$

where we have in addition incorporated the fact that the fermions are left-handed leading one to insert $(1 / 2)\left(1-\gamma_{5}\right)$ in the trace over states, which projects out the right-handed states. This is equivalent to the taking of a difference of terms with antiperiodic and periodic boundary conditions for $\psi$ 's in the path-integral, since $\gamma_{5}=(-1)^{\bar{\psi}_{i} \psi_{i}}$, as can be easily seen by the action of this operator on the states in (7). $\gamma_{5}$ in this basis turns out to be

$$
\gamma_{5}=\left(\begin{array}{cc}
-1 & 0 \\
0 & 1
\end{array}\right)
$$

Notice that there are no additional terms that impose restrictions to specific sectors, as in Section 6. We will again resort to evaluating the integral for an open line and identifying the endpoints at the end, with and without minus signs built in. Thus, for an open line, with $\bar{\lambda}_{m}(1)=\bar{\lambda}_{m 2}$ and $\lambda_{m}(0)=\lambda_{m 1}$, one has

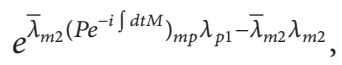

where $M$ has the same meaning as before, and hence adding the boundary contribution and identifying the endpoints as 
$\lambda_{m}(1)=-\lambda_{m}(0)$ for antiperiodic boundary conditions and $\lambda_{m}(1)=\lambda_{m}(0)$ for periodic, one has

$$
\begin{aligned}
& \int d \bar{\lambda} d \lambda e^{-\left(\bar{\lambda}_{m}\left(P e^{-i \int d t M}\right)_{m p} \lambda_{p}+\bar{\lambda}_{m} \lambda_{m}\right)} \\
& +\int d \bar{\lambda} d \lambda e^{-\left(-\bar{\lambda}_{m}\left(P e^{-i \int d t M}\right)_{m p} \lambda_{p}+\bar{\lambda}_{m} \lambda_{m}\right)} \\
& =\operatorname{det}\left(P e^{i \int d t\left(g \dot{x}^{\mu} A_{\mu}^{a} T^{a}+T g \psi^{\mu} F_{\mu \nu}^{a} T^{a} \psi^{\nu}\right)}+1\right) \\
& +\operatorname{det}\left(1-P e^{i \int d t\left(g \dot{x}^{\mu} A_{\mu}^{a} T^{a}+T g \psi^{\mu} F_{\mu \nu}^{a} T^{a} \psi^{\nu}\right)}\right) .
\end{aligned}
$$

This expression is valid for the full $S U(3)_{c} \times S U(2)_{W} \times U(1)_{Y}$ background of the Standard Model. In particular, for $S U(3)_{c}$ the electrons and neutrinos go for free rides in the loops, as they are color singlets.

\section{QCD}

Let us concentrate on QCD. For it, one can consider that $\lambda_{i}$ 's range over 3 values instead of five, and one has the representations

$$
\bar{\lambda}_{i_{1}} \cdots \bar{\lambda}_{i_{p}}|0\rangle
$$

where $p$ ranges from 0 to 3 , with 0 and 3 corresponding to color singlets and the other 2 values corresponding to the fundamental and bifundamental/antifundamental quarks. Now, in general there are other $\bar{\lambda}$ 's also present that correspond to flavor and we are suppressing those as they do not couple to the color gauge bosons (cf. Table 1). The bifundamentals have the fermionic operator content $\bar{\lambda}_{i} \bar{\lambda}_{j}|0\rangle$ and hence correspond to $(3 \otimes 3)_{a s}=\overline{3}$. Since one is considering all the representations, there is no need for a projection operator, and hence the fermionic boundary conditions for $\lambda$ 's are only antiperiodic. However the fermions are still lefthanded, so the other projection operator applies. QCD does not distinguish between left-handed and right-handed, but on the other hand considering a left-handed antiquark is equivalent to considering a right-handed quark, which is why one can work with only left-handed fermions, even in the case of QCD. Thus the effective path-integral over $\lambda$ 's is just

$$
\int d \bar{\lambda} d \lambda e^{-\left(\bar{\lambda}_{m}\left(P e^{-i \int d t M}\right)_{m p} \lambda_{p}+\bar{\lambda}_{m} \lambda_{m}\right)}
$$

This can be easily evaluated by expanding the first term and realizing that the expansion terminates after the third order term. Thus one has [33]

$$
\begin{aligned}
& \int d \bar{\lambda} d \lambda\left(1-\bar{\lambda}_{m}\left(P e^{-i \int d t M}\right)_{m p} \lambda_{p}+\frac{1}{2}\right. \\
& \cdot \bar{\lambda}_{m}\left(P e^{-i \int d t M}\right)_{m p} \lambda_{p} \bar{\lambda}_{m^{\prime}}\left(P e^{-i \int d t M}\right)_{m^{\prime} p^{\prime}} \lambda_{p^{\prime}}-\frac{1}{6} \\
& \cdot \bar{\lambda}_{m}\left(P e^{-i \int d t M}\right)_{m p} \lambda_{p} \bar{\lambda}_{m^{\prime}}\left(P e^{-i \int d t M}\right)_{m^{\prime} p^{\prime}} \\
& \left.\cdot \lambda_{p^{\prime}} \bar{\lambda}_{m^{\prime \prime}}\left(P e^{-i \int d t M}\right)_{m^{\prime \prime} p^{\prime \prime}} \lambda_{p^{\prime \prime}}\right) e^{-\bar{\lambda}_{m} \lambda_{m} .}
\end{aligned}
$$

Let us call $\left(P e^{-i \int d t M}\right)_{m p}, B_{m p}$. Let us first consider the third term. For it, the contribution from the exponential is just 1 (since the terms apart from the exponent completely saturate the measure as for $S U(3)$ there are a total of $3 \lambda$ 's and $3 \bar{\lambda}$ 's, and hence only the first term in the expansion of the exponent, that is, 1 , would contribute in this case) and hence, it is given by

$$
-\frac{1}{6} \epsilon_{m m^{\prime} m^{\prime \prime}} \epsilon_{p p^{\prime} p^{\prime \prime}}\left(B_{m p} B_{m^{\prime} p^{\prime}} B_{m^{\prime \prime} p^{\prime \prime}}\right)=-\operatorname{det} B .
$$

Then the above expression becomes, upon using,

$$
\int d \bar{\lambda} d \lambda\left(\cdots \lambda_{i} \bar{\lambda}_{j} \cdots\right) e^{-\bar{\lambda}_{m} \lambda_{m}}=\left(\cdots \delta_{i j} \cdots\right)
$$

and the expression (220) can be written ${ }^{5}$

$$
1+\operatorname{Tr} B+\frac{1}{2}(\operatorname{Tr} B)^{2}-\frac{1}{2} \operatorname{Tr}\left(B^{2}\right)-\operatorname{det} B .
$$

Note that the naive assumption of quarks transforming in the fundamental representation only would have led to just the second term, and the actual expression is richer. Now since $M$ are traceless and Hermitian, $B$ belongs to the special unitary group and hence $\operatorname{det} B=1$, and hence the full expression just reduces to

$$
\operatorname{Tr} B+\frac{1}{2}(\operatorname{Tr} B)^{2}-\frac{1}{2} \operatorname{Tr}\left(B^{2}\right)
$$

To check whether we have the right expression it might be instructive to work out the trace terms for fundamentals and bifundamentals separately. For fundamentals, one already knows the answer; it is given by $\operatorname{Tr} B$ (cf. (210)). For bifundamentals, the path-integral would be

$$
\begin{aligned}
& \frac{1}{2} \int \frac{d T}{T} \int_{P} \mathscr{D} x\left(\int_{\mathrm{AP}}-\int_{P}\right) \mathscr{D} \psi \mathscr{D} \bar{\psi} \int_{\mathrm{AP}} \mathscr{D} \lambda \mathscr{D} \bar{\lambda} \\
& \quad \cdot \int \mathscr{D} a \times\left(e^{\int_{0}^{1} d t\left(-(1 / 4 T) \dot{x}^{2}+i g \dot{x}^{\mu} \mathrm{A}_{\mu}^{i j} \bar{\lambda}_{i} \lambda_{j}+i T g \psi^{\mu} \mathbf{F}_{\mu \nu}^{i j} \bar{\lambda}_{i} \lambda_{j} \psi^{\nu}-\bar{\psi}_{j} \dot{\psi}_{j}-\bar{\lambda}_{i} \dot{\lambda}_{i}-i a \bar{\lambda}_{i} \lambda_{i}+i(2-3 / 2) a\right)}\right) .
\end{aligned}
$$


Again, doing the $\lambda$ path-integral, one has

$$
\begin{aligned}
& \int d \bar{\lambda} d \lambda \frac{d a}{(2 \pi)} e^{2 i a} e^{-\left(e^{-i a} \bar{\lambda}_{m}\left(T e^{-i \int d t M}\right)_{m p} \lambda_{p}+\bar{\lambda}_{m} \lambda_{m}\right)}=\frac{1}{2 !} \\
& \cdot \int d \bar{\lambda} d \lambda \bar{\lambda}_{m}\left(T e^{-i \int d t M}\right)_{m p} \lambda_{p} \bar{\lambda}_{m^{\prime}}\left(T e^{-i \int d t M}\right)_{m^{\prime} p^{\prime}} \\
& \cdot \lambda_{p^{\prime}} e^{-\bar{\lambda}_{m} \lambda_{m}}=\frac{1}{2}(\operatorname{Tr} B)^{2}-\frac{1}{2} \operatorname{Tr}\left(B^{2}\right)
\end{aligned}
$$

and hence we have essentially reproduced (223).
The contributions from the singlets are trivial and cancel each other out, since $\operatorname{det} B=1$. The full effective action is thus

$$
\begin{aligned}
& \frac{1}{2} \int \frac{d T}{T} \\
& \cdot \int_{P} \mathscr{D} x\left(\int_{\mathrm{AP}}-\int_{P}\right) \mathscr{D} \psi \mathscr{D} \bar{\psi} e^{\int_{0}^{1} d t\left(-(1 / 4 T) \dot{x}^{2}-\bar{\psi}_{j} \dot{\psi}_{j}\right)} \\
& \cdot\left(\operatorname{Tr} P e^{i \int d t\left(g \dot{x}^{\mu} A_{\mu}^{a} T^{a}+\operatorname{Tg} \psi^{\mu} F_{\mu \nu}^{a} T^{a} \psi^{\nu}\right)}\right. \\
& +\frac{1}{2}\left(\operatorname{Tr} P e^{i \int d t\left(g \dot{x}^{\mu} A_{\mu}^{a} T^{a}+\operatorname{Tg} \psi^{\mu} F_{\mu \nu}^{a} T^{a} \psi^{\nu}\right)}\right)^{2} \\
& \left.\quad-\frac{1}{2} \operatorname{Tr}\left(P e^{i \int d t\left(g \dot{x}^{\mu} A_{\mu}^{a} T^{a}+\operatorname{Tg} \psi^{\mu} F_{\mu \nu}^{a} T^{a} \psi^{\nu}\right)}\right)^{2}\right)
\end{aligned}
$$

which can also be written, because of (218), as

$$
\frac{1}{2} \int \frac{d T}{T} \int_{P} \mathscr{D} x\left(\int_{\mathrm{AP}}-\int_{P}\right) \mathscr{D} \psi \mathscr{D} \bar{\psi} e^{\int_{0}^{1} d t\left(-(1 / 4 T) \dot{x}^{2}-\bar{\psi}_{j} \dot{\psi}_{j}\right)} \operatorname{det}\left[P e^{i \int d t\left(g \dot{x}^{\mu} A_{\mu}^{a} T^{a}+T g \psi^{\mu} F_{\mu \nu}^{a} T^{a} \psi^{\nu}\right)}+1\right],
$$

where $T^{a}$ corresponds to the $S U(3)$ group.

\section{Anomalies and Anomaly Cancellations in the Standard Model}

The chiral anomaly is well known to be given by $\operatorname{Tr} \gamma_{5}$, the trace being inclusive of the position and other internal coordinates as well. Since the trace over the position coordinates formally gives rise to infinity while that over $\gamma_{5}$ gives rise to zero, this object needs to be regularized. It is well known [47] that when the regularized trace is performed, one gets

$$
\operatorname{Tr} \gamma_{5}=-\frac{1}{32 \pi^{2}} g^{2} \int d^{4} x \epsilon_{\mu \nu \rho \sigma} \operatorname{Tr}\left[\mathbf{F}^{\mu \nu} \mathbf{F}^{\rho \sigma}\right]
$$

Another avatar of the same thing is the anomaly in $\partial_{\mu}\left\langle J^{\mu 5}\right\rangle$, with $J^{\mu 5}$ being given by $\bar{\Psi}_{i} \gamma^{\mu} \gamma^{5} \Psi_{i}$, where $i$ is the internal index of the gauge group in question, and it is well known that the anomaly is given by

$$
\partial_{\mu}\left\langle J^{\mu 5}\right\rangle=-\frac{1}{16 \pi^{2}} g^{2} \int d^{4} x \epsilon_{\mu \nu \rho \sigma} \operatorname{Tr}\left[\mathbf{F}^{\mu \nu} \mathbf{F}^{\rho \sigma}\right]
$$

where $\mathbf{F}^{\mu \nu}$ is $F_{a}^{\mu \nu} T_{a}$ and $T_{a}$ 's are the gauge group generators. Here the fermions transform in the fundamental of the gauge group. $\operatorname{Tr} \gamma_{5}$ is also well known as the Atiyah-Singer index (for the nongravitational case considered here).

On the other hand when considering the chiral nonAbelian or gauge anomaly in the SM, instead of computing piece by piece, one might try to look for a way of summing over the entire SM spectrum of left-handed states (i.e., the
16), and indeed one can do that, as we saw in the preceding sections, by simply considering [35]

$$
\begin{aligned}
i \eta_{a} & \frac{1}{4} \operatorname{Tr} \gamma_{5}\left(1-\gamma_{5}\right) \hat{\bar{\lambda}}_{i} T_{a i j} \hat{\lambda}_{j}\left(1+(-1)^{\hat{\bar{\lambda}}_{i} \hat{\lambda}_{i}}\right) \\
\quad & i \eta_{a} \frac{1}{4} \operatorname{Tr}_{\mathrm{Cs}} \gamma_{5}\left(1-\gamma_{5}\right) \hat{\bar{\lambda}}_{i} T_{a \mathrm{i} j} \hat{\lambda}_{j}\left(1+(-1)^{\hat{\bar{\lambda}}_{i} \hat{\lambda}_{i}}\right),
\end{aligned}
$$

where the usual projection operators $\left(1-\gamma_{5}\right)$ and $\left(1+(-1)^{\overline{\bar{\lambda}}_{i} \hat{\lambda}_{i}}\right)$ have been introduced that project to a single generation SM spectrum. CS denotes coherent states. Now to start with, on the LHS the "internal" trace is over states of the form $\langle 0| \widehat{\lambda}_{i} \widehat{\lambda}_{j} \cdots \widehat{\psi}_{i}$ in the spin and internal space (cf. Section 6) and the various charges for the various generators $T^{a}$ arise out of the action on these states by $\widehat{\bar{\lambda}}_{i} T_{i j}^{a} \widehat{\lambda}_{j}$ for $S U(3) \times S U(2) \times U(1)$. So when, on the RHS, we pass over to the trace over the coherent states $\langle\lambda, \psi|$, it is $\overline{\bar{\lambda}}_{i} T_{i j}^{a} \widehat{\lambda}_{j}$ that we should continue considering as the generators. Since we are summing over the entire SM spectrum, this must turn out to be identically zero if the SM is to be nonanomalous. In [35], the expression for the gauge anomaly was taken to be computed piece by piece by considering for a fermion transforming as an $\mathbf{n}$

$$
i \eta_{a} \operatorname{Tr} \gamma_{5} \widehat{\bar{\Lambda}}_{i} \widetilde{T}_{a i j} \widehat{\Lambda}_{j}
$$

$\widetilde{T}$ 's being the $n$-dimensional representations of the generators of the SM, with the understanding that one must take the trace over states of the form $\widehat{\bar{\Lambda}}_{i}|0\rangle$ only (with $i$ running over 1 to $n$ ), namely, the $n$-dimensional fundamental representation. Then the total anomaly was supposed to be obtained essentially by summing over the contributions for the different n's that form the states of the SM (cf. Section 6). But here we demonstrate that there is no need to adopt such a piecemeal 
approach and incorporate the different representations of the SM gauge group at once and sum over the entire spectrum, by making use of the GSO-like projectors above, and the result turns out to be zero. In doing this, we are of course committing to the fact that the representations of the SM are given by (109), and this gives further vindication that that is correct.

As is well known [47] the above expressions can be regularized by introducing regulators in the following manner (ignoring the prefactor of $1 / 4$ in the second expression for the time being):

$$
\begin{gathered}
\operatorname{Tr} \gamma_{5} \longrightarrow \lim _{T \rightarrow 0} \operatorname{Tr} \gamma_{5} e^{-T\left[-(\partial+i g \mathbf{A})^{2}-(1 / 2) g \sigma_{\mu v} \mathbf{F}_{\mu v}\right]}, \\
\operatorname{Tr} \gamma_{5}\left(1-\gamma_{5}\right) \hat{\bar{\lambda}}_{i} T_{a i j} \hat{\lambda}_{j}\left(1+(-1)^{\hat{\bar{\lambda}_{i}} \hat{\lambda}_{i}}\right) \longrightarrow \lim _{T \rightarrow 0} \operatorname{Tr}_{C S} \hat{\bar{\lambda}}_{i} T_{a i j} \hat{\lambda}_{j}\left((-1)^{\hat{\bar{\psi}}_{i} \hat{\psi}_{i}}-1\right)\left(1+(-1)^{\hat{\bar{\lambda}}_{i} \hat{\bar{\lambda}}_{i}}\right) e^{-T\left[-(\partial+i g \mathbf{A})^{2}-(i / 2) g \sigma_{\mu v} \mathbf{F}_{\mu v}\right]},
\end{gathered}
$$

where in the first expression the gauge group is $S U(N)$ for some $N$, while, in the second, it is the full SM gauge group. The trace in $x$ 's means one has to again take path-integrals with periodic boundary conditions in them. Analogous to the previous expression (213), the path-integral representations of the above expressions would be

$$
\begin{array}{r}
\lim _{T \rightarrow 0} \int_{P} \mathscr{D} x \int \mathscr{D} a \int_{P} \mathscr{D} \psi \mathscr{D} \bar{\psi} \int_{\mathrm{AP}} \mathscr{D} \lambda \mathscr{D} \bar{\lambda}\left(e^{\int_{0}^{1} d t\left(-(1 / 4 T) \dot{x}^{2}+i g \dot{x}^{\mu} \mathrm{A}_{\mu}^{i j} \bar{\lambda}_{i} \lambda_{j}+i T g \psi^{\mu} \mathrm{F}_{\mu \nu}^{i j} \bar{\lambda}_{i} \lambda_{j} \psi^{\nu}-\bar{\psi}_{j} \dot{\psi}_{j}-\bar{\lambda}_{i} \dot{\lambda}_{i}-i a \bar{\lambda}_{i} \lambda_{i}+i(1-n / 2) a\right)}\right), \\
\lim _{T \rightarrow 0} \int_{P} \mathscr{D} x\left(\int_{\mathrm{AP}}-\int_{P}\right) \mathscr{D} \psi \mathscr{D} \bar{\psi}\left(\int_{\mathrm{AP}}+\int_{P}\right) \mathscr{D} \lambda \mathscr{D} \bar{\lambda} \times\left(\bar{\lambda}_{i} T_{a i j} \lambda_{j} e^{\int_{0}^{1} d t\left(-(1 / 4 T) \dot{x}^{2}+i g \dot{x}^{\mu} \mathrm{A}_{\mu}^{i j} \bar{\lambda}_{i} \lambda_{j}+i T g \psi^{\mu} \mathrm{F}_{\mu \nu}^{i j} \bar{\lambda}_{i} \lambda_{j} \psi^{\nu}-\bar{\psi}_{j} \dot{\psi}_{j}-\bar{\lambda}_{i} \dot{\lambda}_{i}\right)}\right),
\end{array}
$$

respectively.

The difference between the above two expressions is to be noted. In the first expression, one is considering Dirac fermions $\Psi_{i}$ in the fundamental representation of the gauge group and hence the extra $a$-dependent terms in it (as per the above sections). In the second expression, one is summing over even-dimensional $S U(5)$ representations, which together form the $\mathbf{1 6}$ of $S O(10)$, and that is taken care of by the sum over periodic and antiperiodic boundary conditions, and there is no necessity of introducing $a$ dependent terms.

Now the above expressions can be evaluated by taking advantage of the fact that $T \rightarrow 0$ and scaling some of the variables with respect to $T$ in a manner that makes most of the $0+1$-dimensional field theory amplitudes redundant. The details are as in $[36,37]$. In particular, one scales $\psi$ 's by setting $\psi=\psi^{\prime} / \sqrt{2 T}$ and then dropping the prime but leaves $\lambda$ 's and $x$ 's invariant.
In this case, the $x_{\mu}(t)$ field theory can be made redundant by expanding it as $x_{\mu}+q_{\mu}(t)$, where $x_{\mu}$ is the "background" and $q_{\mu}(t)$ is the quantum fluctuation. $q_{\mu}(t)$ Green's functions are proportional to $T$ whereas the vertices are $T$ independent after the scalings and hence all the $q$-dependent contributions coming from $\dot{q}(t) \cdot \mathbf{A}_{\mu}^{i j}(x+q(t)) \bar{\lambda}_{i} \lambda_{j}$ and $\psi^{\mu} \mathbf{F}_{\mu \nu}^{i j}(x+$ $q(t)) \bar{\lambda}_{i} \lambda_{j} \psi^{\nu}$ to the path-integral would simply drop out when the limit $T \rightarrow 0$ is taken. All that the $x_{\mu}(t)$ part then contributes to is the measure $\int d^{4} x$ coming from the integral over the background $x_{\mu}$, the $(1 / 2) \psi \cdot \mathbf{F}_{i j}(x) \cdot \psi \bar{\lambda}_{i} \lambda_{j}$ factor, and the free path-integral over $q_{\mu}(t)$ which contributes a factor $(4 \pi T)^{-2}$, the $(2 T)^{-2}$ part of which is absorbed by the $\psi$ scalings.

Thus the relevant expressions boil down to, ignoring the factor of $1 /(2 \pi)^{2}$, and gauge-fixing $a$,

$$
\lim _{T \rightarrow 0} \int d^{4} x \int \frac{d a}{(2 \pi)} \int_{\mathrm{AP}} \mathscr{D} \lambda \mathscr{D} \bar{\lambda} \int_{P} \mathscr{D} \psi \mathscr{D} \bar{\psi} e^{-\int_{0}^{1} d t\left[\left((1 / 2 T) \bar{\psi}_{j} \dot{\psi}_{j}+\bar{\lambda}_{i} \dot{\lambda}_{i}\right)-i g \bar{\lambda}_{l}\left[(1 / 2) \psi \cdot \mathbf{F}_{l m}(x) \cdot \psi\right] \lambda_{m}-i a \bar{\lambda}_{i} \lambda_{i}+i(1-n / 2) a\right]}
$$

and the corresponding expression for (234). Now the $\psi$ correlators also become proportional to $T$ upon scaling and the vertices become $T$ independent, thereby making its field theory redundant as well, and in what follows we will make heavy use of that. First of all the two different boundary conditions in (234) present two separate ways of going about the problem. In the case of periodic boundary conditions for $\psi_{i}$ 's one can separate them into background and quantum parts, as periodic boundary conditions allow for zero modes, so that even if the quantum part is rendered redundant by the scalings, there is a finite nontrivial contribution coming from the zero modes or the background part. This is essentially because in the measure there are integrals over the zero modes and these need to be saturated, and that comes from the zero modes in the integrand. 
However for the antiperiodic boundary conditions there are no zero modes and the whole $\psi_{i}(t)$ field is quantum and hence rendered redundant by the scalings. And since in expression (234) there are no terms independent of the $\psi_{i}(t)$ in the interaction term after the $x$ dependent terms have been eliminated, the entire path-integral vanishes. Hence we need to bother only with the periodic case.

11.1. The Chiral Anomaly. Here we will assume that the quantum part of $\psi_{i}(t)$ has been rendered redundant and all that remains in the expression is the zero mode which we will again call $\psi_{i}$. The $\lambda$ and $a$ path-integrals can be written as

$$
\begin{aligned}
& \int \frac{d a}{(2 \pi)} \\
& \quad \cdot \int_{\mathrm{AP}} \mathscr{D} \lambda \mathscr{D} \bar{\lambda} e^{-\int_{0}^{1} d t\left[\bar{\lambda}_{i} \dot{\lambda}_{i}-i g \bar{\lambda}_{l}\left[(1 / 2) \psi \cdot \mathbf{F}_{l m}(x) \cdot \psi\right] \lambda_{m}-i a \bar{\lambda}_{i} \lambda_{i}+i(1-n / 2) a\right]} .
\end{aligned}
$$

Going by (208) and (210), this would simply beget

$$
\operatorname{Tr}\left(P e^{-i \int d t M}\right)
$$

where in this case $\int_{0}^{1} d t M=(g / 2) \psi \cdot \mathbf{F} \cdot \psi$. This then reproduces precisely, upon restoring the factor of $1 /(2 \pi)^{2}$ above (235) and the integrals over the $x$ and $\psi$ zero modes, the standard expression $[35,36]$ for the chiral anomaly for fermions, namely,

$$
\frac{1}{(2 \pi)^{2}} \int d^{4} x \int d^{4} \psi \operatorname{Tr}\left(e^{i(g / 2) \psi \cdot \mathbf{F} \cdot \psi}\right)
$$

which simply yields

$$
-\frac{1}{32 \pi^{2}} g^{2} \int d^{4} x \epsilon_{\mu \nu \rho \sigma} \operatorname{Tr}_{R}\left[\mathbf{F}^{\mu \nu} \mathbf{F}^{\rho \sigma}\right]
$$

thus tallying with (228). This can be compared with the derivations in $[36,37]$, to appreciate the economy of arguments and mathematical steps. Note that we hence have also derived the formula for the Atiyah-Singer index for the case at hand.

11.2. The Gauge Anomaly. Let us now deal with the chiral non-Abelian anomaly or gauge anomaly. The expression corresponding to (234) is given by

$$
i \eta_{a} \lim _{T \rightarrow 0} \int_{P} \mathscr{D} x\left(\int_{\mathrm{AP}}-\int_{P}\right) \mathscr{D} \psi \mathscr{D} \bar{\psi}\left(\int_{\mathrm{AP}}+\int_{P}\right) \mathscr{D} \lambda \mathscr{D} \bar{\lambda} \times\left(\bar{\lambda}_{i} T_{a i j} \lambda_{j} e^{\int_{0}^{1} d t\left(-(1 / 4 T) \dot{x}^{2}+i g \dot{x}^{\mu} \mathrm{A}_{\mu}^{i j} \bar{\lambda}_{i} \lambda_{j}+i \operatorname{Tg} \psi^{\mu} \mathbf{F}_{\mu \nu}^{i j} \bar{\lambda}_{i} \lambda_{j} \psi^{\nu}-\bar{\psi}_{j} \dot{\psi}_{j}-\bar{\lambda}_{i} \dot{\lambda}_{i}\right)}\right)
$$

where $T_{a}$ belongs to the SM gauge group and $i$ ranges over 5 values. The $i \eta_{a} \bar{\lambda}_{i} T_{a i j} \lambda_{j}$ term can be exponentiated as follows:

$$
\lim _{T \rightarrow 0} \int_{P} \mathscr{D} x\left(\int_{\mathrm{AP}}-\int_{P}\right) \mathscr{D} \psi \mathscr{D} \bar{\psi}\left(\int_{\mathrm{AP}}+\int_{P}\right) \mathscr{D} \lambda \mathscr{D} \bar{\lambda} \times\left(e^{\int_{0}^{1} d t\left(-(1 / 4 T) \dot{x}^{2}+i g \dot{x}^{\mu} \mathbf{A}_{\mu}^{i j} \bar{\lambda}_{i} \lambda_{j}+i T g \psi^{\mu} \mathbf{F}_{\mu \nu}^{i j} \bar{\lambda}_{i} \lambda_{j} \psi^{\nu}+i \eta_{a} \bar{\lambda}_{i} T_{a i j} \lambda_{j}-\bar{\psi}_{j} \dot{\psi}_{j}-\bar{\lambda}_{i} \dot{\lambda}_{i}\right)}\right)
$$

where the understanding is that the anomaly would be given by the term linear in $\eta_{a}$. The antiperiodic boundary conditions for $\psi$ would not contribute and we need to deal only with the periodic case. And as before, it would boil down to first evaluating

$$
\left(\int_{\mathrm{AP}}+\int_{P}\right) \mathscr{D} \lambda \mathscr{D} \bar{\lambda}\left(e^{\int_{0}^{1} d t\left((i / 2) g \psi^{\mu} \mathrm{F}_{\mu \nu}^{i j} \bar{\lambda}_{i} \lambda_{j} \psi^{\nu}+i \eta_{a} \bar{\lambda}_{i} T_{a i j} \lambda_{j}-\bar{\lambda}_{i} \dot{\lambda}_{i}\right)}\right) .
$$

We would have as in previous sections

$$
\begin{aligned}
& \int d \bar{\lambda} d \lambda e^{-\left(\bar{\lambda}_{m}\left(e^{(i / 2)\left[g \psi \cdot \mathbf{F}(x) \cdot \psi+2 \eta_{a} T_{a}\right]}\right)_{m p} \lambda_{p}+\bar{\lambda}_{m} \lambda_{m}\right)} \\
& +\int d \bar{\lambda} d \lambda e^{-\left(-\bar{\lambda}_{m}\left(e^{(i / 2)\left[g \psi \cdot \mathrm{F}(x) \cdot \psi+2 \eta_{a} T_{a}\right]}\right)_{m p} \lambda_{p}+\bar{\lambda}_{m} \lambda_{m}\right)}
\end{aligned}
$$

$$
\begin{aligned}
= & 2 \int d \bar{\lambda} d \lambda[1 \\
& +\frac{1}{2}\left(\bar{\lambda}_{m}\left(e^{(i / 2)\left[g \psi \cdot \mathbf{F}(x) \cdot \psi+2 \eta_{a} T_{a}\right]}\right)_{m p} \lambda_{p}\right)^{2} \\
& \left.+\frac{1}{4 !}\left(\bar{\lambda}_{m}\left(e^{(i / 2)\left[g \psi \cdot \mathbf{F}(x) \cdot \psi+2 \eta_{a} T_{a}\right]}\right)_{m p} \lambda_{p}\right)^{4}\right] e^{-\bar{\lambda}_{m} \lambda_{m}} .
\end{aligned}
$$

So let us again call the matrix $e^{(i / 2)\left[g \psi \cdot \mathbf{F}(x) \cdot \psi+2 \eta_{a} T_{a}\right]}, B$. As before, one has $\left((\operatorname{Tr} B)^{2}-\operatorname{Tr}\left(B^{2}\right)\right)$ from the second term, and from the fourth

$$
\begin{aligned}
& \frac{1}{12} \int d \lambda d \bar{\lambda}\left(\bar{\lambda}_{m} B_{m p} \lambda_{p} \bar{\lambda}_{m^{\prime}} B_{m^{\prime} p^{\prime}} \lambda_{p^{\prime}} \bar{\lambda}_{m^{\prime \prime}} B_{m^{\prime \prime} p^{\prime \prime}} \lambda_{p^{\prime \prime}} \bar{\lambda}_{m^{\prime \prime \prime}} B_{m^{\prime \prime \prime} p^{\prime \prime \prime}} \lambda_{p^{\prime \prime \prime}}\right) e^{-\bar{\lambda}_{m} \lambda_{m}} \\
& =\frac{1}{12}\left((\operatorname{Tr} B)^{4}-6 \operatorname{Tr}\left(B^{2}\right)(\operatorname{Tr} B)^{2}+3\left(\operatorname{Tr}\left(B^{2}\right)\right)^{2}+8 \operatorname{Tr}\left(B^{3}\right) \operatorname{Tr} B-6 \operatorname{Tr}\left(B^{4}\right)\right) .
\end{aligned}
$$


And, since the trace of $\left[g \psi \cdot \mathbf{F}(x) \cdot \psi+2 \eta_{a} T_{a}\right]$ would vanish, the second term, for instance, in the above expression would look like

$$
\begin{array}{r}
-\frac{1}{2} \operatorname{Tr}\left(1-\frac{i}{3 !} 3\left(2 \eta_{a} T_{a}\right)(g \psi \cdot \mathbf{F}(x) \cdot \psi)^{2}\right) \\
\cdot \operatorname{Tr}\left(1-\frac{i}{3 !} \frac{3}{8}\left(2 \eta_{a} T_{a}\right)(g \psi \cdot \mathbf{F}(x) \cdot \psi)^{2}\right) \\
\cdot \operatorname{Tr}\left(1-\frac{i}{3 !} \frac{3}{8}\left(2 \eta_{a} T_{a}\right)(g \psi \cdot \mathbf{F}(x) \cdot \psi)^{2}\right)
\end{array}
$$

where one has kept terms that are linear in $\eta_{a}$ and quartic in $\psi$ 's in each trace, and the overall coefficient for this term looks like

$$
\begin{array}{r}
i \frac{5}{8} \operatorname{Tr}(1)^{2} \operatorname{Tr}\left(\left(\eta_{a} T_{a}\right)(g \psi \cdot \mathbf{F}(x) \cdot \psi)^{2}\right) \\
\quad=i \frac{125}{8} \operatorname{Tr}\left(\left(\eta_{a} T_{a}\right)(g \psi \cdot \mathbf{F}(x) \cdot \psi)^{2}\right)
\end{array}
$$

since $\operatorname{Tr}(1)=5$, as the indices range over 5 values for $S U(5)$ (which is inherent in the assumptions, since $\lambda$ 's and $\bar{\lambda}$ 's run over five values in order to generate the full SM fermion spectrum).

Now the sum over the coefficients of all such terms in (244) simply vanishes. The 1 would not survive the $\psi$ integration. Doing the remaining integration over $\psi$ 's and factoring out $\eta_{a}$, one simply gets that the anomaly is, ignoring prefactors involving $\pi$ and other numerical coefficients,

$$
\begin{gathered}
(0) g^{2} \int d^{4} x \int d^{4} \psi \operatorname{Tr}\left(T_{a}(\psi \cdot \mathbf{F}(x) \cdot \psi)^{2}\right) \\
=(0) g^{2} \int d^{4} x \epsilon_{\mu \nu \rho \sigma} \operatorname{Tr}\left[T_{a} \mathbf{F}^{\mu \nu} \mathbf{F}^{\rho \sigma}\right]
\end{gathered}
$$

which is the correct expression for the gauge anomaly, with a vanishing coefficient which thus demonstrates the gauge anomaly freedom of the SM, since $T_{a}$ can be any of its generators and one has summed over the full fermionic spectrum. Note that $T_{a}$ and F's in the above expression are in the fundamental representation. Note also that this gives just the leading order term of the anomaly-in that $\mathbf{F}^{\mu \nu}=$ $\partial^{\mu} \mathbf{A}^{\nu}-\partial^{\nu} \mathbf{A}^{\mu}$ only-and not the consistent one [35], which can however be computed via the Wess-Zumino consistency conditions.

Notice that the above expressions could also have been obtained by considering each $\mathbf{n}$ separately by simply incorporating terms of the form $-i a \bar{\lambda}_{i} \lambda_{i}+i(m-5 / 2) a$ in the action, where $\mathbf{n}=5_{C_{m}}$, and it is evident that the above automatically represents the results of taking $m=0,2$ and 4 together, for $\mathbf{n}=\mathbf{1}, \mathbf{1 0}$, and $\overline{\mathbf{5}}$, respectively.

Thus we see that taking a holistic approach and treating all the 16 states of the SM together by simply taking a sum over the periodic and antiperiodic boundary conditions for $\lambda$ 's are a rather economical way of deriving the gauge anomaly freedom of the SM.

\section{Conclusions}

We have thus given representations of propagators for the gauge bosons and chiral fermions of a single generation of the SM (both free and dressed, massless and massive for the fermions) and its fermionic one-loop effective actions and anomalies, based upon the worldline formalism of quantum field theory. Our approach relied upon the use of fermionic worldline fields to represent the additional spin and color information of the particle multiplets, and associated worldline gauge fields to project onto appropriate sectors of the Hilbert space. Such an approach had been initiated in $[13,19,21-23]$ in the context of dressed propagators in the Abelian case and we have extended the formalism to the non-Abelian case (Section 7) (see [12] for related approaches) and made concrete use of the Abelian version in deriving the QED vertex (Section 8). We also applied BRST quantization to verify that the correct gauge fixing procedures had been adopted (Section 4). Since the various SM fields arise as excitations of the quantized worldline theories (Sections 5 and 6), this then gives one the quantum mechanical models, whose wave-functions are the various fields of the SM. String models that try to achieve the same thing have the problem that the wave-functions that arise out of its quantization encompass too many other fields apart from the SM. However, unlike string theory, it is not out of a unique single model that the various fields of the SM arise (we have considered only gauge bosons and fermions), but two different sets of models for the fermions and gauge bosons, and that is a disadvantage.

Our approach also proved, as we said, amenable to the determination of chiral anomalies and demonstrations of part of the cancellations of the gauge ones (Section 11).

With the details discussed in this paper, it would seem appropriate to consider application to specific physical problems; an example, building upon [48], would be to calculate propagators in a constant (non-)Abelian background. One might also contemplate extending the derivation of the QED vertex to the non-Abelian case, as well as to higher point functions, leading to, for instance, the derivation of the Compton scattering amplitude in QED. And then, extending from that point onward, one might think of applying the formalism to compute amplitudes for processes at the LHC.

Mostly, the main purport of the paper is setting up a formalism for doing more advanced calculations relevant for high energy processes. To date, people have mostly dealt with one and higher loop amplitudes in the worldline formalism, skirting the issue of tree amplitudes as it proved to be more formidable. We have taken the first steps here towards the latter program, uncovering the utility of coherent states in possible future applications. However, as we said before, we are currently engaged in building even more powerful methods stemming from all this that give rise to these things much more economically.

The fact that the 3 generations of SM fermions organize themselves as 3 distinct spinors of $S O(10)$ as well as the fact that the explanation of the hypercharge quantum numbers is a natural explanation if the corresponding generator is a diagonal one of $S U(5)$ seem in our opinion, to be tantalizing 
hints that the structure of nature does indeed make use of these groups, especially of $S O(10)$ ( $S U(5)$ being a subgroup). From the structure of the excitations, arrived at through the action of worldline fermionic creation operators, it is tempting to propose that even the higher generations arise via the action of more creation operators on the fields of the first generation. We are also working on such a model, through which we find that there are ramifications on the scalar sector as well, in the sense that the scalar sector is populated with a panoply of scalar fields that couple to the fermions in such a way that it leads to a particularly economical set of couplings (4 or 5) from which everything in the SM could potentially arise. That is, one can essentially trade the arbitrariness in the number of parameters in the SM with a proliferation of the number of scalar fields in an extension of it. However we have to admit that such a model is already hugely constrained by nonoccurrences of such scalars at the LHC, and our hope is that there might be some natural mechanism that automatically renders these scalars much more massive than the Higgs. We believe that the reduction in the arbitrariness of the parameters in the SM is a feature interesting enough to merit attention.

\section{Appendix}

\section{A. Manipulations with Fermionic Path-Integrals}

Here we evaluate the expression

$$
\int_{\lambda(0)=\lambda_{1}}^{\bar{\lambda}(1)=\bar{\lambda}_{2} e^{-i a}} \mathscr{D} \lambda \mathscr{D} \bar{\lambda} e^{i \int_{0}^{1} d t(i \bar{\lambda} \dot{\lambda}-\bar{\lambda}(t) M(t) \lambda(t)+\bar{J}(t) \lambda(t)-J(t) \bar{\lambda}(t))}
$$

by generalizing the methods of [49] from constant $M$ to a time-dependent $\mathrm{M}(t)$. Thus we first define the intermediate time points to be $t_{n}=n \epsilon$ for $n=1,2, \ldots, N-1$, where the infinitesimal time interval is defined to be $\epsilon=1 / N$. We can then write the path-integral to be

$$
\begin{aligned}
& \lim _{N \rightarrow \infty} \widetilde{N} \int d \bar{\lambda}_{1} \cdots d \bar{\lambda}_{N-1} d \lambda_{1} \\
& \quad \cdots d \lambda_{N-1} e^{i \epsilon \sum_{n=1}^{N}\left(i \bar{\lambda}_{n}\left(\left(\lambda_{n}-\lambda_{n-1}\right) / \epsilon\right)-M_{n} \bar{\lambda}_{n}\left(\left(\lambda_{n}+\lambda_{n-1}\right) / 2\right)+\bar{J}_{n} \lambda_{n}-J_{n} \bar{\lambda}_{n}\right)},
\end{aligned}
$$

where $\lambda_{0}=\lambda_{1}$ and $\bar{\lambda}_{N}=\bar{\lambda}_{2} e^{-i a}$. Here one has used the mid-point prescription of Weyl ordering as discussed in [49], along with the fact that the variable $\bar{\lambda}$ represents the momentum conjugate to $\lambda$. The exponent in the above equation can be written out in detail as

$$
\begin{aligned}
& -\left[\sum_{n=1}^{N-1}\left(1+\left(\frac{i \epsilon M_{n}}{2}\right)\right) \bar{\lambda}_{n} \lambda_{n}\right. \\
& +\left(1+\left(\frac{i \epsilon M_{N}}{2}\right)\right) \bar{\lambda}_{N} \lambda_{N} \\
& \quad-\sum_{n=2}^{N-1}\left(1-\frac{i \epsilon M_{n}}{2}\right) \bar{\lambda}_{n} \lambda_{n-1}-\left(1-\frac{i \epsilon M_{1}}{2}\right) \bar{\lambda}_{1} \lambda_{0} \\
& \left.-\left(1-\frac{i \epsilon M_{N}}{2}\right) \bar{\lambda}_{N} \lambda_{N-1}+\bar{J}_{n} \lambda_{n}-J_{n} \bar{\lambda}_{n}\right] .
\end{aligned}
$$

Thus, defining $(N-1)$ component matrices

$$
\begin{gathered}
\lambda=\left(\begin{array}{c}
\lambda_{1} \\
\lambda_{2} \\
\vdots \\
\lambda_{N-1}
\end{array}\right), \\
\bar{\lambda}=\left(\begin{array}{c}
\bar{\lambda}_{1} \\
\bar{\lambda}_{2} \\
\vdots \\
\bar{\lambda}_{N-1}
\end{array}\right),
\end{gathered}
$$$$
J^{\prime}=-\left(1-\frac{i \epsilon M_{1}}{2}\right)\left(\begin{array}{c}
\lambda_{1} \\
0 \\
\vdots \\
0
\end{array}\right),
$$

$$
\bar{J}^{\prime}=-\left(1-\frac{i \in M_{N}}{2}\right)\left(\begin{array}{c}
0 \\
0 \\
\vdots \\
\bar{\lambda}_{N}
\end{array}\right)
$$

we can write the path-integral above as

$$
\begin{aligned}
& \lim _{N \rightarrow \infty} \widetilde{N} \\
& \quad \cdot \int d \bar{\lambda} d \lambda e^{-\left(\bar{\lambda}^{T} B \lambda+\left(\bar{J}^{T}-i \epsilon \bar{J}^{T}\right) \lambda+\bar{\lambda}^{T}\left(-i \epsilon J+J^{\prime}\right)+\left(1+i \epsilon M_{N} / 2\right) \bar{\lambda}_{N} \lambda_{N}\right),}
\end{aligned}
$$

where $B$ is defined as

$$
\left(\begin{array}{ccccc}
x_{1} & 0 & 0 & 0 & \cdots \\
y_{1} & x_{2} & & & \cdots \\
& y_{2} & x_{3} & & \cdots \\
& & y_{3} & x_{4} & \cdots \\
\vdots & \vdots & \vdots & \vdots & \ddots
\end{array}\right)
$$

with

$$
\begin{aligned}
& x_{n}=\left(1+\frac{i \epsilon M_{n}}{2}\right), \\
& y_{n}=-\left(1-\frac{i \epsilon M_{n}}{2}\right) .
\end{aligned}
$$

The path-integral in (A.5) can now be easily evaluated and the result is 


$$
\begin{aligned}
& \lim _{N \rightarrow \infty} \widetilde{N} \operatorname{det} B e^{\left(\left(\bar{J}^{\prime T}-i \epsilon \bar{J}^{T}\right) B^{-1}\left(-i \epsilon J+J^{\prime}\right)-\left(1+i \epsilon M_{N} / 2\right) \bar{\lambda}_{N} \lambda_{N}\right)} \\
& \lim _{N \rightarrow \infty} \widetilde{N} \operatorname{det} B e^{\left(\bar{J}_{N-1}^{\prime} B_{N-1,1}^{-1} J_{1}^{\prime}-i \epsilon \bar{J}_{N-1}^{\prime} B_{N-1, p}^{-1} J_{p}-i \epsilon \bar{J}_{p} B_{p, 1}^{-1} J_{1}^{\prime}-\epsilon^{2} \bar{J}_{p} B_{p, q}^{-1} J_{q}-\left(1+i \epsilon M_{N} / 2\right) \bar{\lambda}_{N} \lambda_{N}\right)} .
\end{aligned}
$$

Now

$$
\begin{aligned}
\operatorname{det} B & =\prod_{n=1}^{N-1} x_{n}=\prod_{n=1}^{N-1}\left(1+\frac{i \epsilon M_{n}}{2}\right)=\prod_{n=1}^{N-1} e^{(i / 2) \epsilon M_{n}} \\
& =T e^{(i / 2) \int d t M(t)} .
\end{aligned}
$$

and it can be easily checked that

$$
\begin{aligned}
B_{p, q}^{-1} & =(-1)^{p-q} x_{q}^{-1} y_{q} x_{q+1}^{-1} y_{q+1} \cdots y_{p-1} x_{p}^{-1} \\
& =\left(1+\frac{i \epsilon M_{q}}{2}\right)^{-1}\left(1-\frac{i \epsilon M_{q}}{2}\right)\left(1+\frac{i \epsilon M_{q+1}}{2}\right)^{-1} \\
& \cdot\left(1-\frac{i \epsilon M_{q+1}}{2}\right) \cdots\left(1-\frac{i \epsilon M_{p-1}}{2}\right) \\
& \cdot\left(1+\frac{i \epsilon M_{p}}{2}\right)^{-1}
\end{aligned}
$$

for

$$
\begin{aligned}
\sum_{n} B_{m n} B_{n p}^{-1}=B_{m, m-1} B_{m-1, p}^{-1}+B_{m, m} B_{m, p}^{-1} \\
=y_{m-1}(-1)^{m-1-p} x_{p}^{-1} y_{p} x_{p+1}^{-1} y_{p+1} \cdots y_{m-2} x_{m-1}^{-1} \\
\quad+x_{m}(-1)^{m-p} x_{p}^{-1} y_{p} x_{p+1}^{-1} y_{p+1} \cdots y_{m-1} x_{m}^{-1} \\
=\delta_{m p} .
\end{aligned}
$$

Thus,

$$
\begin{aligned}
B_{N-1,1}^{-1}= & (-1)^{N-2} x_{1}^{-1} y_{1} x_{2}^{-1} y_{2} \cdots y_{N-2} x_{N-1}^{-1} \\
= & \left(1+\frac{i \epsilon M_{1}}{2}\right)^{-1}\left(1-\frac{i \epsilon M_{1}}{2}\right)\left(1+\frac{i \epsilon M_{2}}{2}\right)^{-1} \\
& \cdot\left(1-\frac{i \epsilon M_{2}}{2}\right) \cdots\left(1-\frac{i \epsilon M_{N-2}}{2}\right) \\
& \cdot\left(1+\frac{i \epsilon M_{N-1}}{2}\right)^{-1} .
\end{aligned}
$$

In the continuum limit of $\epsilon \rightarrow 0$ and $N \rightarrow \infty$, one has

$$
B_{p, q}^{-1}=\theta\left(t_{p}-t_{q}\right) T e^{-i \int_{t_{q}}^{t_{p}} d t M(t)}
$$

the theta function occurring because one has all the matrix elements of $B^{-1}$ lying in the triangular region below or on the diagonal, and hence one has, upon substituting, $J_{1}^{\prime}=-(1-$ $\left.i \epsilon M_{1} / 2\right) \lambda_{1}, \bar{J}_{N-1}^{\prime}=-\left(1-i \epsilon M_{N} / 2\right) \bar{\lambda}_{N}$

$$
\begin{aligned}
& \widetilde{N} T e^{(i / 2) \int d t M(t)} \exp \left[\bar{\lambda}_{N} T e^{-i \int_{0}^{1} d t M(t)} \lambda_{1}\right. \\
& \quad+i \int d t \bar{\lambda}_{N} T e^{-i \int_{t}^{1} d t^{\prime} M\left(t^{\prime}\right)} J(t) \\
& \quad+i \int d t \bar{J}(t) T e^{-i \int_{0}^{t} d t^{\prime} M\left(t^{\prime}\right)} \lambda_{1} \\
& \quad-\int d t \int d t^{\prime \prime} \bar{J}(t) \theta\left(t-t^{\prime \prime}\right) T e^{-i \int_{t^{\prime \prime}}^{t} d t^{\prime} M\left(t^{\prime}\right)} J\left(t^{\prime \prime}\right) \\
& \left.\quad-\bar{\lambda}_{N} \lambda_{N}\right]
\end{aligned}
$$

which upon substituting the values for $\bar{\lambda}_{N}$ is

$$
\begin{aligned}
& \widetilde{N} T e^{(i / 2) \int d t M(t)} \exp \left[\bar{\lambda}_{2} e^{-i a} T e^{-i \int_{0}^{1} d t M(t)} \lambda_{1}\right. \\
& \quad+i \int d t \bar{\lambda}_{2} e^{-i a} T e^{-i \int_{t}^{1} d t^{\prime} M\left(t^{\prime}\right)} J(t) \\
& \quad+i \int d t \bar{J}(t) T e^{-i \int_{0}^{t} d t^{\prime} M\left(t^{\prime}\right)} \lambda_{1} \\
& \quad-\int d t \int d t^{\prime \prime} \bar{J}(t) \theta\left(t-t^{\prime \prime}\right) T e^{-i \int_{t^{\prime \prime}}^{t} d t^{\prime} M\left(t^{\prime}\right)} J\left(t^{\prime \prime}\right) \\
& \left.\quad-\bar{\lambda}_{2} \lambda_{2}\right] .
\end{aligned}
$$

Thus, to summarize, the above is equal to

$$
\int_{\lambda(0)=\lambda_{1}}^{\bar{\lambda}(1)=\bar{\lambda}_{2} e^{-i a}} \mathscr{D} \lambda \mathscr{D} \bar{\lambda} e^{i \int_{0}^{1} d t(i \bar{\lambda} \dot{\lambda}-\bar{\lambda}(t) M(t) \lambda(t)+\bar{J}(t) \lambda(t)-J(t) \bar{\lambda}(t))} .
$$

In particular, when doing perturbation theory for the $\psi$ variables as in Section 8 , if one wants to set $M$ and $a$ to zero, then one has

$$
\begin{aligned}
& Z(J, \bar{J}) \\
& \quad=\int_{\psi(0)=\psi_{1}}^{\bar{\psi}(1)=\bar{\psi}_{2}} \mathscr{D} \psi \mathscr{D} \bar{\psi} e^{i \int_{0}^{1} d t(i \bar{\psi} \dot{\psi}+\bar{J}(t) \psi(t)-J(t) \bar{\psi}(t))+\bar{\psi}(1) \psi(1)} \\
& \quad=\widetilde{N} \exp \left[\bar{\psi}_{2} \psi_{1}+i \int d t \bar{\psi}_{2} J(t)+i \int d t \bar{J}(t) \psi_{1}\right. \\
& \left.\quad-\int d t \int d t^{\prime \prime} \bar{J}(t) \theta\left(t-t^{\prime \prime}\right) J\left(t^{\prime \prime}\right)\right] .
\end{aligned}
$$


Thus if one is interested in the correlators for $\psi$ 's and $\bar{\psi}$ 's, one can take derivatives with respect to $J$ 's and $\bar{J}$ 's and one gets

$$
\begin{aligned}
& \left\langle\bar{\psi}(t) \psi\left(t^{\prime}\right)\right\rangle=\frac{\delta}{\delta J(t)} \frac{\delta}{\delta \bar{J}\left(t^{\prime}\right)} Z(J, \bar{J}) \\
& =\frac{\delta}{\delta J(t)}\left[i \psi_{1}-\int d t^{\prime \prime} \theta\left(t^{\prime}-t^{\prime \prime}\right) J\left(t^{\prime \prime}\right)\right] Z(J, \bar{J}) \\
& =\left[-\theta\left(t^{\prime}-t\right)-\left(i \psi_{1}-\int d t \theta\left(t^{\prime}-t\right) J(t)\right)\right. \\
& \left.\cdot\left(-i \bar{\psi}_{2}+\int d t^{\prime} \bar{J}\left(t^{\prime}\right) \theta\left(t^{\prime}-t\right)\right)\right] Z(J, \bar{J})
\end{aligned}
$$

which yields, upon setting $J(\bar{J})$ 's to zero,

$$
\left\langle\bar{\psi}(t) \psi\left(t^{\prime}\right)\right\rangle=-\left(\theta\left(t^{\prime}-t\right)+\bar{\psi}_{2} \psi_{1}\right) e^{\bar{\psi}_{2} \psi_{1}} \text {. }
$$

When one has multiple fields labelled by $i$ and $j$, one has

$$
\left\langle\bar{\psi}_{i}(t) \psi_{j}\left(t^{\prime}\right)\right\rangle=-\left(\delta_{i j} \theta\left(t^{\prime}-t\right)+\bar{\psi}_{2 i} \psi_{1 j}\right) e^{\bar{\psi}_{2 i} \psi_{1 i}} .
$$

But in this case, one also has, for $i \neq j$,

$$
\begin{aligned}
\left\langle\psi_{i}(t) \psi_{j}\left(t^{\prime}\right)\right\rangle & =-\frac{\delta}{\delta \bar{J}_{i}(t)} \frac{\delta}{\delta \bar{J}_{j}\left(t^{\prime}\right)} Z(J, \bar{J}) \\
& =\left(\psi_{1 i} \psi_{1 j}\right) e^{\bar{\psi}_{2 i} \psi_{1 i}}, \\
\left\langle\bar{\psi}_{i}(t) \bar{\psi}_{j}\left(t^{\prime}\right)\right\rangle & =-\frac{\delta}{\delta J_{i}(t)} \frac{\delta}{\delta J_{j}\left(t^{\prime}\right)} Z(J, \bar{J}) \\
& =\left(\bar{\psi}_{2 i} \bar{\psi}_{2 j}\right) e^{\bar{\psi}_{2 i} \psi_{1 i}} .
\end{aligned}
$$

These correlators are the Dirac versions of the correlators derived for the Majorana fermions in Section 8. Similarly, when one has twisted fields like $\lambda$ 's in Section 6, one gets

$$
\begin{aligned}
& \int_{\lambda(0)=\lambda_{1}}^{\bar{\lambda}(1)=\bar{\lambda}_{2} e^{-i a}} \mathscr{D} \lambda \mathscr{D} \bar{\lambda} e^{i \int_{0}^{1} d t(i \bar{\lambda} \dot{\lambda}+\bar{J}(t) \lambda(t)-J(t) \bar{\lambda}(t))}=\widetilde{N} \\
& \quad \cdot \exp \left[\bar{\lambda}_{2} e^{-i a} \lambda_{1}+i \int d t \bar{\lambda}_{2} e^{-i a} J(t)\right. \\
& \quad+i \int d t \bar{J}(t) \lambda_{1} \\
& \left.\quad-\int d t \int d t^{\prime \prime} \bar{J}(t) \theta\left(t-t^{\prime \prime}\right) J\left(t^{\prime \prime}\right)\right]
\end{aligned}
$$

and, evidently, one would have

$$
\begin{aligned}
& \left\langle\bar{\lambda}_{i}(t) \lambda_{j}\left(t^{\prime}\right)\right\rangle \\
& =-\left(\delta_{i j} \theta\left(t^{\prime}-t\right)+\bar{\lambda}_{2 i} e^{-i a} \lambda_{1 j}\right) e^{\bar{\lambda}_{2 i} e^{-i a}} \lambda_{1 i}, \\
& \left\langle\lambda_{i}(t) \lambda_{j}\left(t^{\prime}\right)\right\rangle=\left(\lambda_{1 i} \lambda_{1 j}\right) e^{\bar{\lambda}_{2 i} e^{-i a} \lambda_{1 i}}
\end{aligned}
$$

as well as

$$
\left\langle\bar{\lambda}_{i}(t) \bar{\lambda}_{j}\left(t^{\prime}\right)\right\rangle=\left(\bar{\lambda}_{2 i} e^{-2 i a} \bar{\lambda}_{2 j}\right) e^{\bar{\lambda}_{2 i} e^{-i a} \lambda_{1 i}}
$$

and when $\bar{J}=J=0$, in (A.9), one essentially has

$$
\begin{aligned}
& \int_{\lambda(0)=\lambda_{1}}^{\bar{\lambda}(1)}=\bar{\lambda}_{2} e^{-i a} \mathscr{D} \lambda e^{i \int_{0}^{1} d t(i \bar{\lambda} \dot{\lambda}-\bar{\lambda}(t) M(t) \lambda(t))} \\
& =\widetilde{N} T e^{(i / 2) \int d t M(t)} e^{\bar{\lambda}_{2} e^{-i a} T e^{-i} \int_{0}^{1} d t M(t)} \lambda_{1}-\bar{\lambda}_{2} \lambda_{2} .
\end{aligned}
$$

When one has multiple fields, the determinant in (A.10) transforms to

$$
\begin{aligned}
& \prod_{n=1}^{N-1} x_{1 n} \prod_{n=1}^{N-1} x_{2 n} \cdots \prod_{n=1}^{N-1} x_{m n}=\prod_{n=1}^{N-1} \prod_{i=1}^{m} x_{i n} \\
& =\prod_{n=1}^{N-1}\left(1+\frac{i \epsilon \sum_{i=1}^{m} M_{i n}}{2}\right)=T e^{(i / 2) \sum_{i} \int d t M_{i}(t)} .
\end{aligned}
$$

\section{B. Computation of Normalization Factors}

Here we specialize to the case of $M_{i}(t)=b$ and consider the path-integral

$$
\int_{\lambda(0)=\lambda_{1}}^{\bar{\lambda}(1)=\bar{\lambda}_{2}} \mathscr{D} \lambda \mathscr{D} \bar{\lambda} e^{i \int_{0}^{1} d t\left(i \bar{\lambda}_{i} \dot{\lambda}_{i}-\bar{\lambda}_{i} b \lambda_{i}+b(m-n / 2)\right)+\bar{\lambda}_{i} \lambda_{i}(1)}
$$

which going by (A.26) and (A.27) is

$$
\widetilde{N} e^{i b(m-n / 2)} e^{(i / 2) n b} e^{\bar{\lambda}_{2 i} e^{-i b} \lambda_{1 i}}=\widetilde{N} e^{i b m} e^{\bar{\lambda}_{2 i} e^{-i b} \lambda_{1 i}}
$$

which is the same as, modulo the normalization factor and the integral over $b$, the expression in (104), thus vindicating its derivation from (103).

Also (B.1) is equivalent to the expression [29]

$$
\left\langle\bar{\lambda}_{2}\left|e^{-i b\left(\hat{\bar{\lambda}}_{i} \hat{\lambda}_{i}-m\right)}\right| \lambda_{1}\right\rangle .
$$

This can be seen as follows. The above is equivalent to

$$
e^{i b m} e^{-i b \bar{\lambda}_{2 i}\left(\partial / \partial \bar{\lambda}_{2 i}\right)}\left\langle\bar{\lambda}_{2} \mid \lambda_{1}\right\rangle=e^{i b m} e^{-i b \bar{\lambda}_{2 i}\left(\partial / \partial \bar{\lambda}_{2 i}\right)} e^{\bar{\lambda}_{2 i} \lambda_{1 i}} .
$$

But the operator $e^{-i b \bar{\lambda}_{2 i}\left(\partial / \partial \bar{\lambda}_{2 i}\right)}$ just generates a finite rescaling of the coordinate $\bar{\lambda}_{2 i} \rightarrow e^{-i b} \bar{\lambda}_{2 i}$ in any function $f\left(\bar{\lambda}_{2 i}\right)$. Hence the above expression is equivalent to (B.2), modulo the normalization factor. Hence it is also equal to (B.1). The equivalence can also be easily established by inserting complete sets of coherent states $\int d \bar{\lambda}_{n i} d \lambda_{n i}\left|\lambda_{n}\right\rangle\left\langle\bar{\lambda}_{n}\right| e^{-\bar{\lambda}_{n i} \lambda_{n i}}$ all over the time interval from 0 to 1 in (B.3). All this then justifies (65) and the first equation in (104).

\section{Some Calculational Details Pertaining to Section 9}

Here we prove

$$
\begin{aligned}
& T\left(e^{(i / 2) \int d t \sum_{i} M_{i}^{\prime}(t)}\right) \prod_{i} e^{\bar{\lambda}_{i 2}^{\prime} e^{-i a} T\left(e^{-i \int d t M_{i}^{\prime}(t)}\right) \lambda_{i 1}^{\prime}} \\
& =e^{e^{-i a} \bar{\lambda}_{m 2}\left(T e^{-i \int d t M}\right)_{m p} \lambda_{p 1} .}
\end{aligned}
$$

First of all (cf. (202)) 


$$
\begin{aligned}
& \sum_{n} M_{n}^{\prime}(t) \\
& \quad=\sum_{n}\left(U_{n i}^{-1}(t) \frac{d}{d t} U_{i n}(t)+i U_{n k}^{-1}(t) M_{k l} U_{l n}(t)\right) .
\end{aligned}
$$

Since the matrices $U(t)$ are special unitary the trace of the derivative term in the above equation vanishes, and so does the other term, due to the cyclicity property of the trace and the tracelessness of $M$, and one is left with

$$
\begin{aligned}
e^{\sum_{n} \bar{\lambda}_{n 2}^{\prime}\left(e^{-i a} T e^{-i \int d t M_{n}^{\prime}(t)}\right)_{n n} \lambda_{n 1}^{\prime}} & =e^{\sum_{n} \bar{\lambda}_{m 2} U(1)_{m n}\left(e^{-i a} T e^{-i \int d t M_{n}^{\prime}(t)}\right)_{n n} U^{-1}(0)_{n p} \lambda_{p 1}} \\
& =e^{e^{-i a} \sum_{n} \bar{\lambda}_{m 2}\left(U(1)\left(e^{-i \epsilon M_{n}^{\prime}(1)} U^{-1}(1-\epsilon) U(1-\epsilon) e^{-i \epsilon M_{n}^{\prime}(1-\epsilon)} U^{-1}(1-2 \epsilon) U(1-2 \epsilon) e^{-i \epsilon M_{n}^{\prime}(1-2 \epsilon)} \ldots\right) U^{-1}(0)\right)_{m p} \lambda_{p 1}} \\
& =e^{e^{-i a} \sum_{n} \bar{\lambda}_{m 2}\left(U(1)\left(e^{-i \epsilon M_{n}^{\prime}(1)}\left(U^{-1}(1)-\epsilon(d / d t) U^{-1}(1)\right) U(1-\epsilon) e^{-i \epsilon M_{n}^{\prime}(1-\epsilon)}\left(U^{-1}(1-\epsilon)-\cdots\right) U(1-2 \epsilon) \cdots\right) U^{-1}(0)\right)_{m p} \lambda_{p 1}} \\
& =e^{e^{-i a} \sum_{n} \bar{\lambda}_{m 2}\left(U(1)\left(e^{-i \epsilon M_{n}^{\prime}(1)} U^{-1}(1)\left(1-\epsilon U(1)(d / d t) U^{-1}(1)\right) U(1-\epsilon) e^{-i \epsilon M_{n}^{\prime}(1-\epsilon)}\left(U^{-1}(1-\epsilon)-\cdots\right) U(1-2 \epsilon) \cdots\right) U^{-1}(0)\right)_{m p} \lambda_{p 1}} \\
& =e^{e^{-i a} \sum_{n} \bar{\lambda}_{m 2}\left(\left(e^{-i \epsilon U(1) M_{n}^{\prime}(1) U^{-1}(1)-\epsilon U(1)(d / d t) U^{-1}(1)}\right)\left(e^{-i \epsilon U(1-\epsilon) M_{n}^{\prime}(1-\epsilon) U^{-1}(1-\epsilon)-\epsilon U(1-\epsilon)(d / d t) U^{-1}(1-\epsilon)}\right) \cdots\right)_{m p} \lambda_{p 1}}
\end{aligned}
$$

which, using (204), is

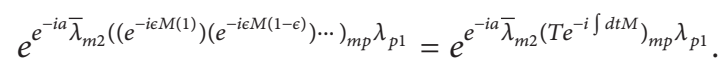

\section{Conflicts of Interest}

The author would like to declare that there are no conflicts of interest regarding the publication of this paper.

\section{Acknowledgments}

The author would like first and foremost to thank James Edwards for various comments and suggestions for improving the paper. He would then like to thank Christian Schubert for correspondence and general encouragement. Thanks are due to his colleagues at New Alipore College for supporting him in various ways. Finally, he would also like to thank the late Binayak Dutta-Roy for general discussions and encouragement.

\section{Endnotes}

1. Note that $\psi_{i}(t=0)=\psi_{1 i}$ and $\bar{\psi}_{i}(t=1)=\bar{\psi}_{2 i}$, and hence $\delta \psi(t)$ vanishes at $t=0$ but not at $t=1$.

2. Consider the eigenstates of the number operator $\widehat{N}|n\rangle=$ $n|n\rangle$, where $\widehat{N}=\widehat{\bar{\psi}}^{\mu} \widehat{\psi}_{\mu}$. Then the operator

$$
\begin{aligned}
\int_{0}^{2 \pi} d a e^{i a(\widehat{N}-s)} & =\int_{0}^{2 \pi} \sum_{n=1}^{N} e^{i a(\widehat{N}-s)}|n\rangle\langle n| \\
& =\sum_{n=1}^{N} \int_{0}^{2 \pi} e^{i a(n-s)}|n\rangle\langle n| \\
& =\sum_{n=1}^{N} \delta_{n, s}|n\rangle\langle n|=\delta_{\widehat{N}, s}
\end{aligned}
$$

where we have assumed that $s$ is an integer and, of course, that the number operator has integer eigenvalues; furthermore, the exchange of sum and integration is guaranteed by the finite sum and compact interval. The last line follows because the previous line is the operator $\delta_{\widehat{N}, s}$ written in the occupation number basis. Thanks to James Edwards for pointing this out.

3. Thanks to James Edwards for pointing this out.

4. The author would like to thank James Edwards for pointing this out.

5. Note that this highlights one of the benefits of the worldline formalism: in maintaining gauge invariance, the result of the path integral has automatically been organised into products of gauge invariant traces.

\section{References}

[1] R. P. Feynman, "Mathematical formulation of the quantum theory of electromagnetic interaction," Physical Review, vol. 80, no. 3, pp. 440-457, 1950.

[2] Z. Bern and D. A. Kosower, "Efficient calculation of one-loop polarized QCD amplitudes," in Proceedings of the Polarized Collider Workshop, pp. 358-363, University Park, 1991.

[3] Z. Bern and D. A. Kosower, "Color decomposition of oneloop amplitudes in gauge theories," Nuclear Physics. B. Theoretical, Phenomenological, and Experimental High Energy Physics. Quantum Field Theory and Statistical Systems, vol. 362, no. 1-2, pp. 389-448, 1991.

[4] Z. Bern and D. A. Kosower, "The computation of loop amplitudes in gauge theories," Nuclear Physics. B. Theoretical, Phenomenological, and Experimental High Energy Physics. Quantum Field Theory and Statistical Systems, vol. 379, no. 3, pp. 451$561,1992$.

[5] M. J. Strassler, "Field theory without Feynman diagrams: oneloop effective actions," Nuclear Physics. B. Theoretical, Phenomenological, and Experimental High Energy Physics. Quantum Field Theory and Statistical Systems, vol. 385, no. 1-2, pp. 145-184, 1992. 
[6] M. G. Schmidt and C. Schubert, "Worldline Green functions for multiloop diagrams," Physics Letters B, vol. 331, no. 1-2, pp. 6976, 1994.

[7] M. Reuter, M. G. Schmidt, and C. Schubert, "Constant external fields in gauge theory and the spin 0,1/2,1 path integrals," Annals of Physics, vol. 259, no. 2, pp. 313-365, 1997.

[8] C. Schubert, "Perturbative quantum field theory in the stringinspired formalism," Physics Reports. A Review Section of Physics Letters, vol. 355, no. 2-3, pp. 73-234, 2001.

[9] O. Corradini and C. Schubert, "Spinning Particles in Quantum Mechanics and Quantum Field Theory," https:// arxiv.org/abs/1512.08694.

[10] F. Bordi and R. Casalbuoni, "Dirac propagator from path integral quantization of the pseudoclassical spinning particle," Physics Letters. B, vol. 93, no. 3, pp. 308-312, 1980.

[11] M. Henneaux and C. Teitelboim, "Relativistic quantum mechanics of supersymmetric particles," Annals of Physics, vol. 143, no. 1, pp. 127-159, 1982.

[12] N. V. Borisov and P. P. Kulish, "Path integral in superspace for a relativistic spinor particle in an external gauge field," Theoretical and Mathematical Physics, vol. 51, no. 3, pp. 535-540, 1982.

[13] V. Y. Fainberg and A. V. Marshakov, "Local supersymmetry and Dirac particle propagator as a path integral," Nuclear Physics. B. Theoretical, Phenomenological, and Experimental High Energy Physics. Quantum Field Theory and Statistical Systems, vol. 306, no. 3, pp. 659-676, 1988.

[14] J. C. Henty, P. S. Howe, and P. K. Townsend, "Quantum mechanics of the relativistic spinning particle," Classical and Quantum Gravity, vol. 5, no. 5, pp. 807-823, 1988.

[15] G. P. Korchemsky, "Quantum geometry of Dirac fermions," Physics Letters. B. Particle Physics, Nuclear Physics and Cosmology, vol. 232, no. 3, pp. 334-344, 1989.

[16] J. Grundberg, T. H. Hansson, and A. Karlhede, "Geometrical phases from spinning particles," Physical Review D, vol. 41, no. 8, pp. 2642-2644, 1990.

[17] G. P. Korchemsky, "Quantum geometry of Dirac fermions," International Journal of Modern Physics A. Particles and Fields. Gravitation. Cosmology, vol. 7, no. 2, pp. 339-380, 1992.

[18] J. Grundberg, T. H. Hansson, and A. Karlhede, “On Polyakov's spin factors," Nuclear Physics. B. Theoretical, Phenomenological, and Experimental High Energy Physics. Quantum Field Theory and Statistical Systems, vol. 347, no. 1-2, pp. 420-440, 1990.

[19] E. S. Fradkin and D. M. Gitman, "Path-integral representation for the relativistic particle propagators and BFV quantization," Physical Review. D. Particles and Fields. Third Series, vol. 44, no. 10, pp. 3230-3236, 1991.

[20] J. W. van Holten, "Propagators and path integrals," Nuclear Physics. B. Theoretical, Phenomenological, and Experimental High Energy Physics. Quantum Field Theory and Statistical Systems, vol. 457, no. 1-2, pp. 375-407, 1995.

[21] D. M. Gitman and S. I. Zlatev, "Spin factor in the path integral representation for the Dirac propagator in external fields," Physical Review. D. Third Series, vol. 55, no. 12, pp. 7701-7714, 1997.

[22] D. M. Gitman, "Path integrals and pseudoclassical description for spinning particles in arbitrary dimensions," Nuclear Physics. B. Theoretical, Phenomenological, and Experimental High Energy Physics. Quantum Field Theory and Statistical Systems, vol. 488, no. 1-2, pp. 490-512, 1997.
[23] T. M. Aliev, V. Y. Fainberg, and N. K. Pak, "Path integral for spin: a new approach," Nuclear Physics. B. Theoretical, Phenomenological, and Experimental High Energy Physics. Quantum Field Theory and Statistical Systems, vol. 429, no. 2, pp. 321-343, 1994.

[24] M. Pierri and V. O. Rivelles, "BRST quantization of spinning relativistic particles with extended supersymmetries," Physics Letters B, vol. 251, no. 3, pp. 421-426, 1990.

[25] S. K. Wong, "Field and particle equations for the classical YangMills field and particles with isotopic spin," Il Nuovo Cimento A, vol. 65, no. 4, pp. 689-694, 1970.

[26] A. P. Balachandran, P. Salomonson, B.-S. Skagerstam, and J.-O. Winnberg, "Classical description of a particle interacting with a non-Abelian gauge field," Physical Review D, vol. 15, no. 8, pp. 2308-2317, 1977.

[27] A. Barducci, R. Casalbuoni, and L. Lusanna, "Classical scalar and spinning particles interacting with external Yang-Mills fields," Nuclear Physics, Section B, vol. 124, no. 1, pp. 93-108, 1977.

[28] R. Fresneda and D. Gitman, "Pseudoclassical description of scalar particle in non-abelian background and path-integral representations," International Journal of Modern Physics A. Particles and Fields. Gravitation. Cosmology, vol. 23, no. 6, pp. 835-853, 2008.

[29] N. Ahmadiniaz, F. Bastianelli, and O. Corradini, "Erratum: dressed scalar propagator in a non-abelian background from the worldline formalism (physical review D - particles, fields, gravitation and cosmology (2016) 93 (025035))," Physical Review D - Particles, Fields, Gravitation and Cosmology, vol. 93, no. 4, Article ID 049904, 2016.

[30] F. Bastianelli, R. Bonezzi, O. Corradini, and E. Latini, "Particles with non abelian charges," Journal of High Energy Physics, no. 10, 098, front matter+12 pages, 2013.

[31] O. Corradini and J. P. Edwards, "Mixed symmetry tensors in the worldline formalism," High Energy Physics - Theory, 2016, https://arxiv.org/abs/1603.07929.

[32] J. P. Edwards and O. Corradini, "Mixed symmetry Wilson-loop interactions in the worldline formalism," Journal of High Energy Physics, vol. 1609, no. 9, 2016.

[33] P. Mansfield, "The fermion content of the Standard Model from a simple world-line theory," Physics Letters, Section B: Nuclear, Elementary Particle and High-Energy Physics, vol. 743, pp. 353356, 2015.

[34] J. P. Edwards, "Unified theory in the worldline approach," Physics Letters B, vol. 750, pp. 312-318, 2015.

[35] L. Alvarez-Gaum'e and E. Witten, "Gravitational anomalies," Nuclear Physics. B. Theoretical, Phenomenological, and Experimental High Energy Physics. Quantum Field Theory and Statistical Systems, vol. 234, no. 2, pp. 269-330, 1984.

[36] J. de Boer, B. Peeters, K. Skenderis, and P. van Nieuwenhuizen, "Loop calculations in quantum mechanical non-linear sigma models with fermions and applications to anomalies," Nuclear Physics. B. Theoretical, Phenomenological, and Experimental High Energy Physics. Quantum Field Theory and Statistical Systems, vol. 459, no. 3, pp. 631-692, 1996.

[37] F. Bastianelli and P. van Nieuwenhuizen, Path integrals and anomalies in curved space, Cambridge Monographs on Mathematical Physics, Cambridge University Press, Cambridge, 2006.

[38] F. A. Berezin and M. S. Marinov, "Particle spin dynamics as the grassmann variant of classical mechanics," Annals of Physics, vol. 104, no. 2, pp. 336-362, 1977.

[39] L. Brink, P. di Vecchia, and P. Howe, "A Lagrangian formulation of the classical and quantum dynamics of spinning particles," Nuclear Physics. B, vol. 118, no. 1-2, pp. 76-94, 1977. 
[40] R. Casalbuoni, "The classical mechanics for bose-fermi systems," Il Nuovo Cimento A Series 11, vol. 33, no. 3, pp. 389-431, 1976.

[41] A. Barducci, R. Casalbuoni, and L. Lusanna, "Supersymmetries and the pseudoclassical relativistic electron," Il Nuovo Cimento A Series 11, vol. 35, no. 3, pp. 377-399, 1976.

[42] P. Howe, S. Penati, M. Pernici, and P. K. Townsend, "A particle mechanics description of antisymmetric tensor fields," Classical and Quantum Gravity, vol. 6, no. 8, pp. 1125-1140, 1989.

[43] F. Bastianelli, P. Benincasa, and S. Giombi, "Worldline approach to vector and antisymmetric tensor fields," Journal of High Energy Physics. A SISSA Journal, no. 4, 010, 27 pages, 2005.

[44] F. Bastianelli, R. Bonezzi, O. Corradini, and E. Latini, "Effective action for higher spin fields on (A)dS backgrounds," Journal of High Energy Physics, vol. 2012, no. 12, article no. 113, 2012.

[45] R. N. Mohapatra and B. Sakita, "SO(2n) Grand Unification in an SU(N) Basis," Physical Review. D. Particles and Fields. Third Series, vol. 21, no. 4, pp. 1062-1066, 1980.

[46] N. Ahmadiniaz, A. Bashir, and C. Schubert, "Multiphoton amplitudes and generalized Landau-Khalatnikov-Fradkin transformation in scalar QED," Physical Review D - Particles, Fields, Gravitation and Cosmology, vol. 93, no. 4, Article ID 045023, 2016.

[47] K. Fujikawa, "Path integral for gauge theories with fermions," Physical Review. D. Particles and Fields. Third Series, vol. 21, no. 10, pp. 2848-2858, 1980.

[48] A. Ahmad, N. Ahmadiniaz, O. Corradini, S. P. Kim, and C. Schubert, "Master formulas for the dressed scalar propagator in a constant field," Nuclear Physics B, vol. 919, pp. 9-24, 2017, arXiv:1612.02944, arXiv.

[49] A. K. Das, "Field theory: A Path integral approach," World Scientific Lecture Notes in Physics, vol. 52, no. 1, 1993. 

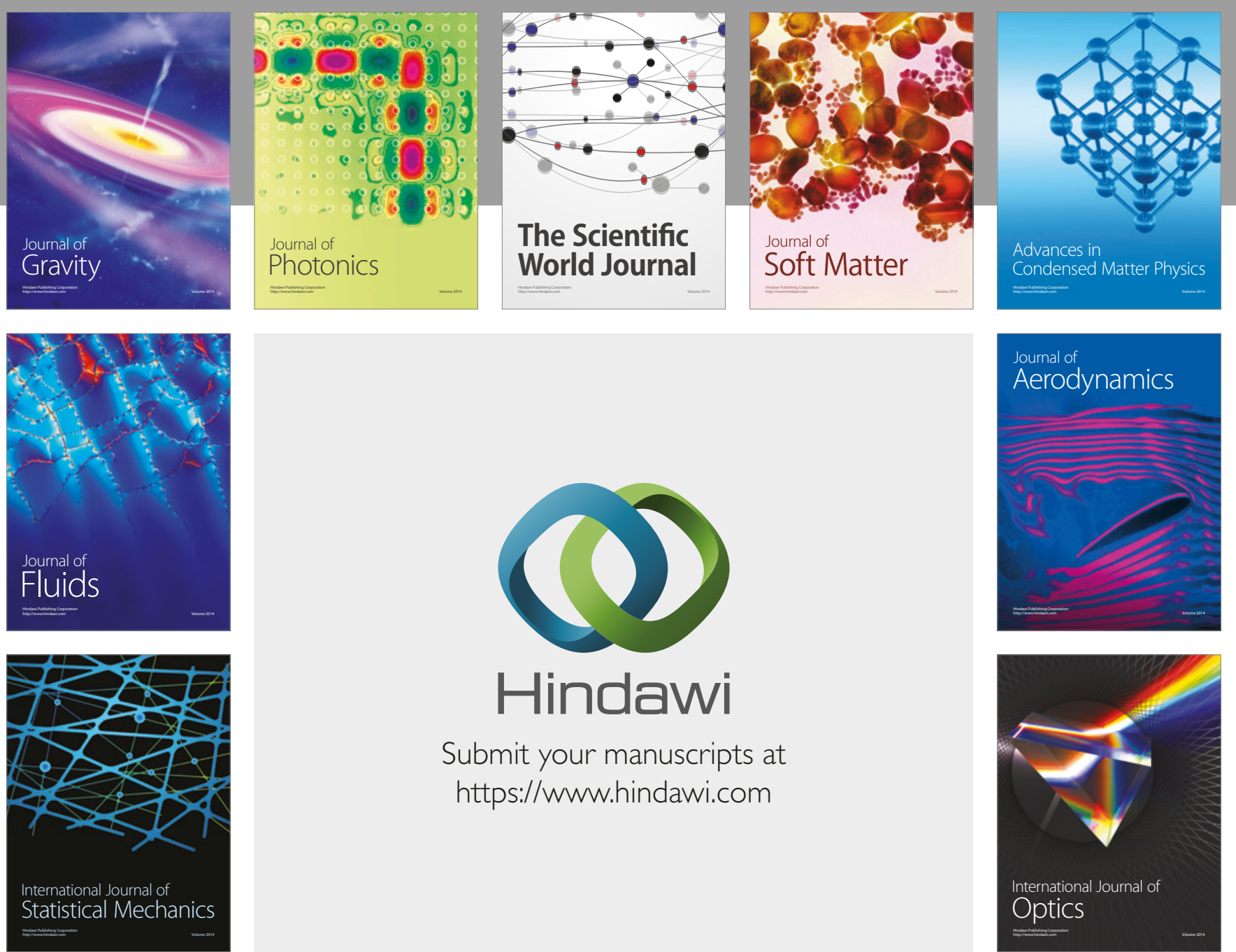

Submit your manuscripts at

https://www.hindawi.com
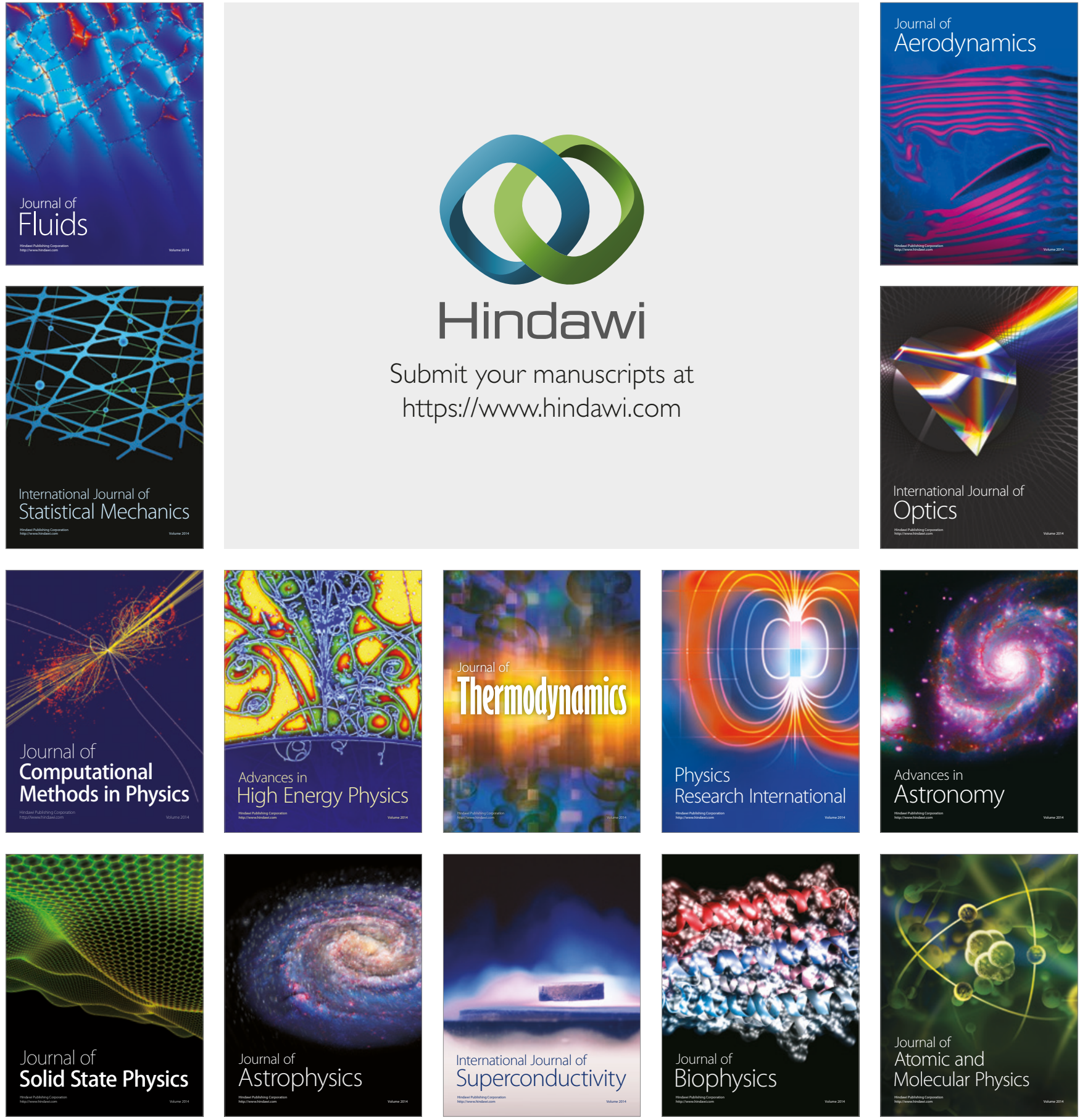